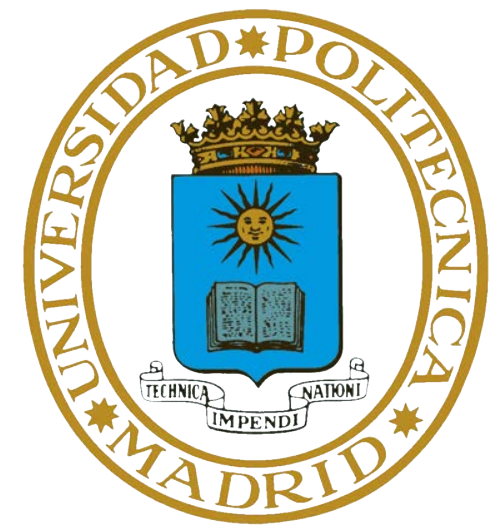

ESCUELA TÉCNICA SUPERIOR DE INGENIEROS INFORMÁTICOS

Lineage Inference of Packed Malware using Binary Code Similarity

\author{
PH.D THESIS
}

Irfan U1 Haq 

Copyright (c) 2019 by Irfan Ul Haq 
DEPARTAMENTAMENTO DE LENGUAJES Y SISTEMAS INFORMÁTICOS E INGENIERIA DE SOFTWARE

ESCUELA TÉCNICA SUPERIOR DE INGENIEROS INFORMÁTICOS

\title{
Lineage Inference of Packed Malware using Binary Code Similarity
}

SUBMITTED IN PARTIAL FULFILLMENT OF THE REQUIREMENTS FOR THE DEGREE OF: Doctor of Philosophy in Software, Systems and Computing

\author{
Author: Irfan Ul Haq \\ Advisor: Dr. Juan Caballero
}

September 2019

Tribunal:

Chair/Presidente: Juan Manuel Estévez Tapiador, Professor, Universidad Carlos III, Spain

Secretary/Secretario: Alessandra Gorla, Assistant Research Professor, IMDEA

Software Institute, Spain

Member/Vocal: Martina Lindorfer, Assistant Professor, Echnische Universität Wien, Austria

Member/Vocal: Ricardo Julio Rodríguez Fernández, Assistant Professor, Universidad de Zaragoza, Spain

Member/Vocal: Marco Guarnieri, Assistant Research Professor, IMDEA Software Institute, Spain 


\section{Abstract of the Dissertation}

Software evolves to adapt to changing requirements by adding new functionality and improving stability through bug fixing. Software lineage studies the evolutionary relationships among software. In particular, program lineage studies the evolution of a program over time across its different versions. The source code of a program manifests its evolution. Unfortunately, many software programs are distributed as executables without source code, e.g., malware and commercial off-the-shelf (COTS) programs. Fortunately, the binary code of a program also reflects its evolution across versions, e.g., added, removed, and updated binary code. Thus, to infer lineage we can leverage binary code similarity approaches that compare two, or more, pieces of binary code and capture their differences.

Similar to benign programs, malware families also evolve over time. But, malware lineage inference has three additional challenges not addressed by existing lineage approaches. First, malware development typically comprises of an extra step not present in benign software development. Once a new version of a malware family is ready, the malware authors pack the resulting executable to hide its original binary code. The only directly visible binary code in a packed malware sample is the code for unpacking the original binary code. Second, the packing process is typically applied many times to the same input executable, creating polymorphic variants of exactly the same malware version. These variants look different to malware detectors, but have the same functionality. Consequently, many packed malware samples may correspond to the same version. Thus, version identification is a fundamental challenge to infer lineage of malware collected in the wild. Third, the development model used by the malware, e.g., straight-line or branching and merging, is unknown. Thus, we need a lineage inference algorithm that works independently of the development model used by the malware family.

In this thesis, we develop an approach for inferring the lineage of packed malware using binary code similarity. In particular, this thesis provides four contributions. First, we perform a systematic study to analyze existing research on binary code similarity. We systematize four aspects of binary code similarity research: the applications enabled, the approaches employed, how the approaches have been implemented, and the benchmarks and evaluation methodologies. 
Second, we propose the first approach to identify malware versions of a malware family. Our approach uses static and dynamic analysis to recover the original binary code of a packed malware and disassemble the recovered binary code. Then, it performs binary code similarity using the semantic hashes of the disassembled code to identify the versions of the malware family.

Third, we develop a malware lineage inference algorithm for packed malware that works independently of the development model of the malware family. It produces a lineage graph where nodes are versions of the family and edges describe the relationships between versions.

Finally, we contribute two metrics to evaluate malware unpacking and disassembly approaches, and use them to evaluate our approach. 


\section{Resumen de la Disertación}

El software evoluciona para adaptarse a los requisitos cambiantes al agregar nuevas funcionalidades y mejorar la estabilidad a través de la corección de errores. El linaje de software estudia las relaciones evolutivas entre software. En particular, el linaje del programa estudia la evolución de un programa a lo largo del tiempo en sus diferentes versiones. El código fuente de un programa manifiesta su evolución. Desafortunadamente, muchos programas de software se distribuyen como ejecutables sin código fuente, por ejemplo, malware y programas comerciales estándar (COTS). Sin embargo, el código binario de un programa también refleja su evolución a través de versiones, por ejemplo, código binario agregado, eliminado y actualizado. Por lo tanto, para calcular el linaje, podemos aprovechar la similitud del código binario mediante la comparacion de dos o más piezas de código binario y capturar sus diferencias.

$\mathrm{Al}$ igual que los programas benignos, las familias de malware también evolucionan con el tiempo. No obstante, la inferencia de linaje de malware tiene tres desafíos adicionales que los enfoques de linaje existentes no abordan. Primero, el desarrollo de malware generalmente comprende un paso adicional que no está presente en el desarrollo de software benigno. Una vez que una nueva versión de una familia de malware está lista, los autores del malware empaquetan el ejecutable resultante para ocultar su código binario original. El único código binario directamente visible en una muestra de malware empaquetada es el código para desempaquetar el código binario original. En segundo lugar, el proceso de empaquetado generalmente se aplica muchas veces al mismo ejecutable de entrada, creando variantes polimórficas de exactamente la misma versión de malware, que parece diferente según los detectores de malware, pero tiene la misma funcionalidad. En consecuencia, muchas muestras de malware empaquetadas pueden corresponder a la misma versión. Por lo tanto, la identificacin de la versión de malware es un desafío fundamental para inferir el linaje de malware recolectado en un enterno real. En tercer lugar, se desconoce el modelo de desarrollo, por ejemplo, línea recta o ramificación y fusión. Una familia de malware puede usar un modelo de desarrollo. Por lo tanto, necesitamos un algoritmo de inferencia de linaje que funcione independientemente del modelo de desarrollo utilizado por la familia de malware.

En esta tesis, desarrollamos un enfoque para inferir el linaje del malware empa- 
quetado utilizando similitud de código binario. En particular, esta tesis proporciona cuatro contribuciones. Primero, realizamos un estudio sistemático para analizar la investigación existente sobre código binario semejante. Sistematizamos cuatro aspectos de la investigación de similitud de código binario: aplicaciónes permitidas, enfoques empleados, cómo se han implementado los enfoques, y puntos de referencia y metodologías de evaluación.

Segundo, proponemos el primer enfoque para identificar versiones de malware de una familia. Nuestro enfoque utiliza análisis estático y dinámico para recuperar el código binario original de un malware empaquetado y desmontar el código binario recuperado. Luego, realiza similitudes de código binario utilizando los hashes semánticos del código desmontado para identificar las versiones de la familia de malware.

Tercero, desarrollamos un algoritmo de inferencia de linaje de malware para malware empaquetado que funciona independientemente del modelo de desarrollo de la familia de malware. Produce un gráfico de linaje donde los nodos son versiones de la familia y los bordes describen las relaciones entre versiones.

Finalmente, contribuimos con dos métricas para evaluar los enfoques de desempaque todo y desensamblado de malware, y los usamos para evaluar nuestro enfoque. 
A mi familia

(To my family) 


\section{Acknowledgments}

This $\mathrm{Ph} . \mathrm{D}$ dissertation bears my name as author but many people have contributed directly and indirectly to its creation. I would like to pay my thanks to those individuals for their support and help.

First and foremost, I would like to pay my gratitude to my supervisor Juan Caballero for given me the opportunity to join his research group. Juan is an inspiring leader and has a very energetic personality. His dedication towards his students and passion for scientific research is admirable. I am grateful to him for allowing me to explore and pursue my research ideas. It was an amazing learning experience working under your supervision.

I would also like to thank to my office mates, Srdjan Maric and Richard Rivera. I have spent most of my time with them. I loved the way Srdjan talked to his programs and his passionate arguments on writing clean and quality code. Richard taught me some basic Spanish to survive my initial days in Spain. Platon Kotzias helped me with thesis defense process. Lastly, Silvia helped me in writing the thesis abstract in Spanish. I am also thankful to the members of Pakistani student community in Madrid, particularly, Umer Liqat, Syed Alam, and Abrar Baloch.

Also, this is the right time to thank my coauthors Somesh Jha and Sergio Chica. Particularly, Somesh Jha for his guidance and valuable feedback on lineage work. I would also like to thank IMDEA Software Institute's administration and support staff. They help in every possible way to maintain a conducive research environment.

Finally, I extend my heartiest gratitude to my family particularly to my beloved mother. She had never attained any form of formal education and never attended any educational system. Yet she had the desire and vision to invest in education. I still remember those days when my family needed me to find a job so that I could provide some support in maintaining everyday subsistence. Despite of knowing our humble background, my mother resisted the idea. She wanted me to continue my education. She worked hard to make sure that I had enough funding available to keep forwarding my studies. I will remain indebted to her for everything she has done for me. 
1 Introduction $\quad 1$

1.1 Research Contributions . . . . . . . . . . . . . . . . . . 4

1.2 Thesis Outline . . . . . . . . . . . . . . . . 5

2 Related Work 7

2.1 Malware Lineage . . . . . . . . . . . . . . . . . . 7

2.2 Malware Unpacking . . . . . . . . . . . . . . . . . . . . 9

2.3 Disassembly . . . . . . . . . . . . . . . . . . . 10

3 A Systematization of Binary Code Similarity 12

3.1 Introduction . . . . . . . . . . . . . . . . . . . 12

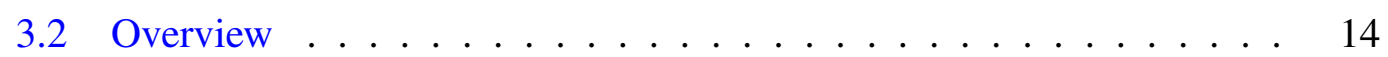

3.2.1 Compilation Process . . . . . . . . . . . . . . 14

3.2.2 Binary Code Similarity Overview . . . . . . . . . . . . . 15

3.3 Scope \& Paper Selection . . . . . . . . . . . . . . . . . . . . 19

3.4 Binary Code Similarity Evolution . . . . . . . . . . . . . 20

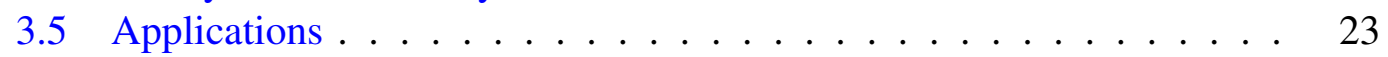

3.6 Approaches .............................. 24

3.6.1 Comparison Type . . . . . . . . . . . . . . . . 24

3.6 .2 Granularity ........................ 25

3.6 .3 Syntactic Similarity . . . . . . . . . . . . . . . 26

3.6 .4 Semantic Similarity . . . . . . . . . . . . . . . . . . . 27

3.6 .5 Structural Similarity . . . . . . . . . . . . . . . . . . . 29

3.6 .6 Feature-Based Similarity . . . . . . . . . . . . . 31

3.6 .7 Hashing . . . . . . . . . . . . . . . . . 32

3.6 .8 Supported Architectures . . . . . . . . . . . . . . . . . 34

3.6 .9 Type of Analysis . . . . . . . . . . . . . . . . . . . . . . 34

3.6 .10 Normalization . . . . . . . . . . . . 35

vii 
3.7 Implementations . . . . . . . . . . . . . . . . . . 36

3.8 Evaluations . . . . . . . . . . . . . . . . . . . . 39

3.8 .1 Datasets . . . . . . . . . . . . . . 39

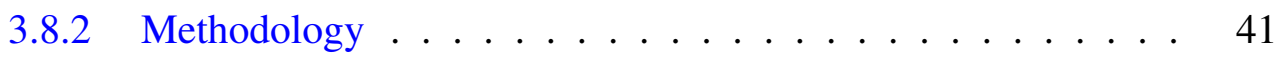

3.9 Discussion . . . . . . . . . . . . . . . . . . 42

4 Lineage Inference of Packed Malware 45

4.1 Introduction . . . . . . . . . . . . . . . . . . . 45

4.2 Overview \& Problem Definition . . . . . . . . . . . . . 48

4.2 .1 Challenges .................... 48

4.2 .2 The Lineage Graph . . . . . . . . . . . . . . . . . . . . . . . 49

4.2 .3 Identifying Malware Versions . . . . . . . . . . . . . 50

4.3 Approach Overview . . . . . . . . . . . . . . . . . 51

4.4 Unpacking . . . . . . . . . . . . . . . . . 53

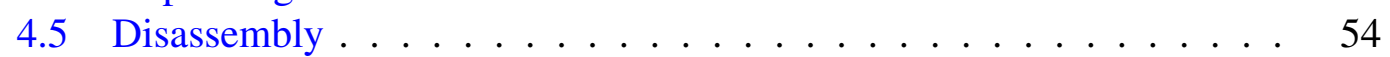

4.6 Lineage Inference . . . . . . . . . . . . . . . . . . . 56

4.6.1 Phase I: Identifying Versions . . . . . . . . . . . . . 56

4.6.2 Phase II: Building a Lineage Tree . . . . . . . . . . . . 57

4.6.3 Phase III: Adding Cross-Edges . . . . . . . . . . . . . . . 58

4.6 .4 Complexity Analysis . . . . . . . . . . . . . . . 59

4.7 Evaluation on Benign Programs . . . . . . . . . . . . . . . 60

4.7 .1 Accuracy Metrics . . . . . . . . . . . . . . . 60

4.7.2 Unpacking \& Disassembly Accuracy . . . . . . . . . . 62

4.7.3 Lineage Inference Accuracy . . . . . . . . . . . . . . 63

4.8 Evaluation on Malware . . . . . . . . . . . . . . . . . 67

4.8.1 Unpacking and Disassembly . . . . . . . . . . . . . . 67

4.8.2 Lineage Inference . . . . . . . . . . . . . . . . . 69

4.9 Discussion . . . . . . . . . . . . . . . . 71

5 Conclusions \& Future Work $\quad \mathbf{7 2}$

5.1 Conclusions . . . . . . . . . . . . . . . 72

5.2 Future Work . . . . . . . . . . . . . . . 73

$\begin{array}{lr}\text { Bibliography } & \mathbf{7 8}\end{array}$ 


\section{List of Figures}

3.1 The extended compilation process. Dotted boxes are optional code transformations typically used for obfuscation. For a given source code, changing any of the grey boxes may produce a different binary program. . . . . . . . . . . . . . .

3.2 Similarity at a finer granularity can be used to infer a different type of similarity at a coarser granularity. . . . . . . . . . . . 16

3.3 Two alternative methods for feature-based similarity. . . . . . . . . 31

4.1 PiCsys lineage graph built using 131 samples. The node label represents the number of distinct functions in the version and number of samples of that version. The edge label is the number of shared func-

4.2 Approach overview. The execution recording and IDA tool are off-theshelf components not developed in this work. . . . . . . . . 51

4.3 PUTTY lineage graph. Cross-edges are marked with asterisk. . . . . 63

4.4 FZPUtTyGen lineage graph. . . . . . . . . . . . . . . 63

4.5 Lineage graph for PUTTY when each version is packed with a different packer. . . . . . . . . . . . . . 66

4.6 Lineage graph for SYTRO built using 1,354 samples. The node label indicates the number of functions in the version and the number of samples of that version. The edge label is the number of shared functions. 


\section{List of Tables}

3.1 Comparison among binary code similarity approaches. For boolean columns $\checkmark$ means supported and $\boldsymbol{X}$ unsupported. Input Comparison can be one-to-one (OO), one-to-many (OM), or many-to-many (MM). Input Granularity and Approach Granularities can be instruction (I), basic block (B), function (F), or program (P). Approach Comparison can be Similar (S), Identical (I), or Equivalent (E). Structural similarity can use CFG (C), ICFG (I), Callgraph (G), and other custom graphs (O). Machine learning can be Supervised (S) or Unsupervised (U). In Normalization, $\boldsymbol{X}$ means no normalization, $\diamond$ operand removal, - operand normalization, o mnemonic normalization, and $\star$ code elimination. . . . . . . . . . . . . . . . .

3.2 Applications evaluated in the analyzed binary code similarity research works. . . . . . . . . . . . . . . 22

3.3 Comparison of the implementations of binary code similarity approaches. Symbol - means information is unknown. . . . . . . . . . . 37

3.4 Comparison of the evaluations of binary code similarity approaches. On the left it summarizes the datasets used and on the right the evaluation methodology. A dash (-) means we could not find the information. For Boolean columns, $\boldsymbol{X}$ indicates no support. . . . . . . . . . . .

4.1 Unpacking and disassembly accuracy evaluation on all SPEC CPU 2006 C programs with SPP-NOP, each packed with 18 packers. For each packer it shows the number of programs evaluated, function coverage, and function noise ratio. . . . . . . . . . . 
4.2 Lineage inference evaluation on 13 open-source programs. For each program, it first shows the earliest and last versions analyzed. Then, the expected lineage graph type ( $S$ for straight-line, $2-S$ for two straight lines, and $D$ for DAG), the number of releases, and the number of expected binary versions (i.e., ground truth). Next, for the lineage graph generated using each hash: the number of nodes $(|V|)$ and root nodes $(|R|)$, the number of cross-edges $(|X|)$, and the partial order (PO) agreement. The next two columns show the PO agreement using ILINE's DAG and straight-line (SL) algorithms. The final column shows the number of versions identified using BINDIFF. . . . . . . .

4.3 Unpacking and disassembly results on packed malware. For each family, it shows the executables analyzed, instructions (in millions) traced for all malware processes, malware processes traced; waves for all malware processes, unpacking runtime, number of unpacked functions in the IDA databases, and disassembly runtime. . . . . . . . . . . .

4.4 Lineage graph details. For each hash, it details the number of versions, edges, the maximum number of samples in a version, the number of singleton versions representing a single sample, the maximum and minimum number of functions in a version, and the total number of functions across all versions. . . . . . . . . . .

5.1 Comparison of the binary analysis platforms used in the implementation of binary code similarity approaches. This table overlaps with previous work on type inference [1]. We have added following six platforms: Angr, Beaengine, Cuckoo, NewBasic, McSema, Vulcan. 


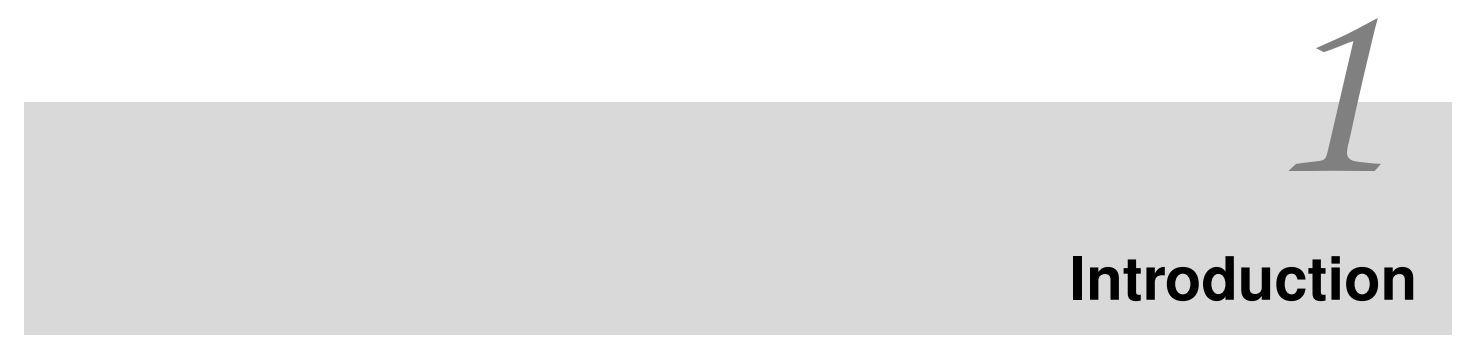

Malicious programs (also referred as malware) play a central role in carrying out cybercrime. In 1986, two brothers in Pakistan developed Brain, a computer program that replaced the original boot sector of a storage media with a custom boot sector (Brain is regarded as the first known virus for MS-DOS). The primary motive of the authors was to protect the copyright infringement of their proprietary medical software. However, the idea was picked, and soon after programs started appearing with malicious intentions such as PC-Write trojan (1986) and programs that highlighted security flaws such as the Morris worm (1988). Since then, malware has evolved in complexity and sophistication $[2,3]$. Today, malware is used to perform a wide range of malicious tasks including keystroke logging, credential theft, online extortion, distributed denial of service (DDoS), cyber espionage, compromising data integrity, and state-sponsored attacks on adversaries critical infrastructure.

The amount of malware distributed has increased significantly over the past decade [46]. During 2018, Symantec observed on average 20.5 million unique malware samples (i.e., having different file hashes) each month [7]. And, the VirusTotal file analysis Web service receives 46 million distinct submissions everyday [6]. But, this sheer volume in number of malware samples or suspicious files does not mean that all the observed variants are unique in terms of functionality. A majority of those samples are packed versions of the same malware program; and tweaked versions of previously seen malware programs.

Software lineage studies the evolutionary relationships among software. In particular, program lineage studies the evolution of a program over time across its different versions. The goal of program lineage is to produce a lineage graph where nodes are versions of a program and edges describe the ancestor-descendant relationships between versions. Program lineage has many important security applications in the context of malware analysis [8]. For example, lineage can be a fundamental step for triage, labeling, categorization, threat intelligence, provenance, and authorship attribution.

Similar to benign programs, malware families evolve to adapt to changing require- 
ments by adding new functionality, and to improve stability by fixing bugs. But, malware development typically comprises of an extra step not present in benign software development. Once a new version of a malware family is ready, the malware authors pack the resulting executable to hide its functionality and thus bypass detection by commercial malware detectors. The packing process takes as input an executable and produces another executable with the same functionality. The packing process is typically applied many times to the same input executable, creating polymorphic variants of exactly the same program version, which look different to malware detectors, but have the same functionality. Packed malware samples are difficult to analyze as unpacking routines decompress/decrypt the original binary code of the malware at runtime [9-13].

Source code version control systems use text diffing utilities, such as UNIX diff, to keep track of different versions by capturing the changes from an old version to an updated version, e.g., added, removed, and tweaked source code lines. Similar to the source code, the binary code of a program also reflects its evolution across different versions, e.g., added, removed, and updated binary code. Binary code similarity approaches compare two, or more, pieces of binary code and identify their differences based on their syntax (e.g., sequences of disassembled instructions), structure (e.g., callgraph, control flow graph), and semantics (e.g., inputs and outputs). Previous approaches have used binary code similarity for recovering the lineage of programs that were not packed $[14,15]$. However, the packing process in malware development hides the original binary code of a malware. Instead, the only directly visible binary code in a packed malware is the packer code for unpacking the original binary code at execution. Lineage inference of packed malware requires of a specialized approach. In this thesis, we develop an approach for inferring the lineage of packed malware using binary code similarity.

Malware lineage. The study of malware lineage goes back over 20 years and multiple approaches have been proposed to address it $[8,14-24]$. However, a persistent limitation of these approaches is that they consider every input sample a separate malware version. Thus, their output lineage graphs have a node for every input sample. This is problematic because the packing process produces many polymorphic variants of a malware version. Thus, many input samples should be represented by the same node in the lineage graph. An important open problem in malware lineage is identifying the versions of a malware family among a set of packed input executables belonging to the family. This problem does not manifest in prior malware lineage approaches because they are evaluated on synthetic malware, malware that is not packed, or packed malware for which unpackers are readily available (e.g., UPX [25]). But, the majority of malware is packed and malware often uses custom packers for which off-the-shelf unpackers are not available [9]. Thus, version identification is a fundamental challenge to infer lineage of malware collected in the wild.

Identifying versions among packed samples from a malware family is a novel and challenging problem. This problem differs from program similarity [26] because two 
versions of the same family may be highly similar, but still different versions. For example, one version may patch the previous one by adding a conditional to fix an error condition. While the two versions may be nearly identical, they need to be represented by different nodes in the lineage graph because both versions are semantically different. We address this problem by considering malware samples with equivalent functions as polymorphic variants, which should be represented by the same node in the lineage graph. Any changes in functionality between two samples such as adding a function, removing a function, or updating an existing function, means that both samples are from different versions, with their own nodes in the lineage graph.

Another challenge with malware collected in the wild is that we do not know how it was developed. The development model of a program can be classified in three categories: straight-line (SL), k-straight lines (k-SL), and branching and merging [15]. SL lineage means each version in the lineage graph has only one parent (except the root version) and only one child (except the last version). A special case of straightline lineage is k-straight lines (k-SL) which has multiple independent straight-lines of development. Multiple lines may happen due to source code refactoring. On the other hand, branching and merging means each version may have multiple parents and multiple children in the lineage graph. It captures the dependence of a version on multiple development branches or it shows that a version is the base of multiple later versions. Therefore, branching and merging development requires the program lineage to be a direct acyclic graph (DAG). The challenge is that the development model used by a malware family is unknown. Thus, we need a lineage inference algorithm that works independently of the development model selected by the malware authors, i.e., straight-line, multiple independent lines, branching and merging.

Unfortunately, ILINE [15], the state-of-the-art lineage inference algorithm for binary code, has three main drawbacks - we discuss other related approaches and their drawbacks in Chapter 2. First, ILINE assumes that each input sample is a node in the lineage graph, i.e., a different version. However, with packed malware, many samples may be variants of the same version. In reality, we first need to identify the malware versions before we can recover the lineage of packed malware. Second, ILINE does not support packed malware. ILINE's input consists of benign programs that are not packed; malware samples for which an unpacker is available; malware samples compiled from leaked source code; and synthetically produced malware samples. However, nowadays most malware samples collected in the wild are packed with unknown and custom packers [9] and distributed without source code and debugging symbols. Third, ILINE uses separate algorithms for straight-line and branching and merging development. Thus, ILINE requires knowing in advance the development model of the software to select the lineage algorithm. This is problematic with malware since the development model is not known.

In this thesis, we develop a system that takes packed malware samples from the same malware family as an input, identifies malware samples corresponding to the same version, and builds a lineage graph of the identified versions. 
Binary code similarity on packed malware. Simply applying existing binary code similarity on packed malware samples will be of little help because the statically visible binary code in an executable corresponds to the unpacking code rather than to the original binary code. This may lead to conclude false similarity. We explain this with the help of an example. Consider that we want to compute similarity between two malware samples, i.e., both malware belong to different malware families, packed with the same packer like PECompact [27], We may conclude that both malware samples are the same because the only directly visible code are PECompact's unpacking routines. Similarly, if we use different packers, e.g., PECompact [27] and Petite [28], to protect the same version of a malware, this may result in no similarity because the packers use different unpacking routines. Thus, a naive application of existing binary code similarity approaches will not work, and we need to develop binary code similarity approaches that can compute binary code similarity of packed malware.

This thesis contributes a binary code similarity approach that uses static and dynamic analysis to recover the original binary code of a packed malware, disassembles the recovered binary code, and finally computes similarity between packed malware. It is important to note that we focus on packed malware samples, but, our techniques are equally applicable to obfuscated benign binary programs. The motivation behind protecting benign programs is to hamper reverse engineering to protect proprietary algorithms and to curb binary code reuse attacks. The reason we focus on malicious programs is that packing is prevalent in malware. Our approach can be equally applied to compare packed benign binary programs as we will show by performing an experiment with benign programs protected with multiple packers.

Accuracy metrics for malware unpacking. Another question we address in this thesis is: how do we measure the accuracy of the unpacking and disassembly process? Most prior unpacking works evaluate their tools qualitatively by showing that the detection rate of $\mathrm{AV}$ engines improves on the unpacked code [12, 13, 29]. But, such evaluation does not quantitatively evaluate the unpacking output. A few works compare the hash of the unpacked code to identify duplicate samples [12,30]. However, such Boolean evaluation (i.e., equal / not equal) does not address cases where not all original code is recovered. No prior unpacking approach proposes metrics to measure the quality of the unpacking output. Quoting a recent work: "we recognize that we have not been able to define a metric that allow us to adequately determine code coverage" [10]. Accuracy metrics are necessary to quantify how good the unpacking is with respect to a ground truth and to compare different approaches. In this thesis, we propose two metrics to evaluate the accuracy of the unpacking and disassembly process, and use them to evaluate our approach.

\subsection{Research Contributions}

Overall, this thesis contributes on binary code similarity of packed binary programs and its application to inferring lineage of packed malware collected from the wild. In 
particular, this thesis provides the following contributions:

- We systematize binary code similarity research. We perform a systematic study to analyze existing research on binary code similarity. We systematize four aspects of binary code similarity research: (1) the applications enabled, (2) the approaches employed, (3) how the approaches have been implemented, and (4) the benchmarks and evaluation methodologies.

- We propose the first approach to identify versions of a malware family. We present the first approach to identify malware versions among packed malware samples. We employ our developed unpacking and disassembly approach to cluster malware versions based on the semantic hashes of their binary code.

- We develop a lineage inference algorithm for packed malware that does not depend on prior information about the development model. We devise an algorithm to find malware lineage on obfuscated malware programs that belong to the same malware family. Our algorithm works independently of the development model used, as such information is rarely available for malware.

- We present two metrics to measure the effectiveness of malware unpacking and disassembly approaches. The lack of meaningful unpacking and disassembly evaluation metrics has been a persistent problem. To this end, we propose two accuracy metrics: Function Coverage (FC) and Function to Noise Ratio (FNR). We employ these metrics to evaluate our unpacking and disassembly approach.

\subsection{Thesis Outline}

The remainder of the thesis is organized as follows.

Chapter 2 discusses the related work on malware lineage, malware unpacking, and disassembly.

Chapter 3 provides a comprehensive survey of research on binary code similarity. We detail the basics of binary code, analyze the binary code generation pipeline and perform a detailed systematization. We focus on four aspects of binary code similarity. First, we describe the evolution of binary code similarity over time and highlight the main events that triggered various research directions. Second, we provide a detailed analysis of binary code similarity approaches. This analysis covers the type of comparison and many aspects of syntactic, semantic, and structural similarity. Next, we explore how the approaches have been implemented. Lastly, we discuss the evaluation benchmarks and evaluation methodologies.

Chapter 4 details our approach for recovering the lineage of packed malware collected from the wild. First, we describe the unpacking and disassembly modules that use dynamic and static analysis to recover the original binary code of a packed malware. 
Then, we detail our technique for malware version identification. Next, we present an algorithm for recovering the lineage of the identified malware versions. We also describe two metrics for measuring the accuracy of malware unpacking and disassembly approaches.

Finally, Chapter 5 discusses the future work and concludes the thesis. 


\section{Related Work}

This chapter describes related approaches on malware lineage, malware unpacking, and disassembly. We defer the discussion of binary code similarity approaches to our survey in Chapter 3.

\subsection{Malware Lineage}

The study of malware lineage goes back over 20 years to the pioneering work of Sorkin [16] and the phylogeny of the Stoned boot sector computer virus by Hull [17]. Most previous research on malware lineage uses a distance-based hierarchical clustering approach that produces a phylogenetic tree $[8,19-21,23]$. Since phylogenetic trees cannot handle multiple ancestors for a node, other approaches such as by Goldberg et al. [18] and ILINE [15] use a DAG. The above approaches analyze malware samples, but lineage can also analyze textual metadata in online threat libraries [22].

There are two fundamental differences between our approach and all prior work in malware lineage. (1) We want to evaluate on real malware samples, which may be packed. All above approaches evaluate on unpacked malware, malware for which unpackers are available (e.g., UPX [25]), and assume unpacking behavior that many state-of-the-art packers have invalidated. For instance, BEAGLE [31] takes memory snapshot at process exit or at the end of analysis duration. Advanced packers, such as PEProtector, replace the original binary code of a program with random data, or with nops, at the end of original program execution. In addition, Ugarte et al. [9] have shown that all the original binary code of a packed malware may not be available at one point in time during malware execution. (2) We do not know apriori the versions of a malware family, i.e., the set of nodes in the lineage graph. Instead, we classify input samples into versions and identify polymorphic variants of the same version. In addition to those two differences, there are another three differences between our work and ILINE, the current state-of-the-art for malware lineage. (3) Our lineage graph is at a different granularity. Rather than using features such as n-grams, individual basic blocks, or API calls observed during execution, we focus on comparing malware 
samples at the level of individual unpacked functions. This enables identifying what functions are added and removed between two versions and how a version is derived from its predecessors. (4) We assume no apriori knowledge of how the malware was developed and use the same approach regardless of the development model (straightline, $k$ independent lines, and branching and merging). (5) Malware disassembly is a challenging task, which needs addressing, e.g., ILINE bypasses it by compiling the synthetic malware's source using $\mathrm{gCC}_{\mathrm{C}}-\mathrm{S}$ to generate the assembly ground truth.

BEAGLE [31] is another approach that studies the malware evolution. Unlike our approach, BEAGLE does not infer lineage of a malware family automatically and it does not study the problem of malware version identification. Rather, BEAGLE compares binary code and quantifies differences among the versions of a malware family, and automatically characterizes the evolution using behavioral analysis, i.e., assigns semantics to the evolution. BEAGLE uses the auto-update functionality of malware samples to collect subsequent versions of a malware family. It indicates that the collected malware samples and their order in evolution somewhat is known apriori. Our approach, on the other hand, automatically produce a lineage graph for a malware family and is oblivious to the order of evolution of the malware versions, i.e., malware samples are collected from the wild.

Lineage evaluation. A related line of work addresses the problem of how to evaluate malware lineage approaches given the lack of ground truth. Hayes et al. [32] reviewed five malware lineage approaches and their evaluation. They propose using artificial malware history generators to create a reference dataset. They propose two techniques for variants generation: mutation-based and feature-accretion. In the mutation-based approach, a real world malware sample from the wild is used as a seed program and then various random code mutations are applied to produce new artificial malware variants with tracking of their evolution. In the feature-accretion approach, a piece of source code is divided into functionality components (also functionality features) and then new variants are produced with extended functionality by adding new features like enabling stealth or starting the program as a service. Those malware variants and their evolution are used as a reference dataset for evaluating malware lineage approaches. Although this approach can produce large set of arbitrary phylogenetic models, it does not capture real world malware and software complexities. Dumitras and Neamtiu [33], on the other hand, propose evaluating on open-source software. They argue the use of open-source programs and their release and development histories to fine-tune lineage approaches. We followed their suggestion and first evaluate the accuracy of our approach on open-source software, before applying it to malware.

Another problem of lineage evaluation is: how do we compare the output of lineage algorithm and a reference graph (i.e., ground truth)? Earlier work [32] use Nodal Distance [34] and standard deviation to measure lineage accuracy. ILINE [15] proposes seven lineage accuracy metrics. They conclude that Partial Order (PO) is a suitable accuracy metric. We report PO for our approach and compare it with ILINE. 


\subsection{Malware Unpacking}

The first case of encrypting the original binary code was found in the Cascada virus in 1986 [35] . It employed symmetrical XOR cipher to encrypt its original code and inserted a new code stub (also called decrypter) to decrypt the original code at runtime. This whole process is referred to as malware packing. Malware packing adds an additional challenge for malware analysts to unpack the malware sample first in order to obtain its original binary code for the analysis. In the literature, malware unpacking approaches can be divided into two categories: static unpacking and generic unpacking.

Static unpacking. Packer identification tools $[36,37]$ use signatures to identify if a program is obfuscated with a specific packer. Those tools can be used to select a static unpacker, if available. PEiD [36], discontinued, detects 470 different signatures and supports PE files. Similarly, RDG [37] supports more than 5,000 signatures for PE $32 / 64$ bit files. However, most of the malware samples in the wild use custom packing routines for which, generally, a static unpacker is not available [9].

Generic unpacking. Generic unpackers have been proposed to avoid manually building static unpackers for each packer and its custom variants [10-13,30]. PolyUnpack [30] identifies the original code entry point (OEP) when a sequence of instructions not present in the program's disassembly is executed. Determining whether a program has finished unpacking is an undecidable problem [30]. Thus, PolyUnpack only applied the analysis to malware executions for a limited time (a few minutes). Christodorescu et al. [29] and Renovo [12] propose the write-then-execute property to identify unpacking, used by many unpackers. Renovo also introduces the concept of multiple code waves (or simply waves), which are created by writing (i.e., unpacking) new code into memory and then transferring execution to that unpacked code. Code waves were later formalized by Debray et al. [38] and Guizani et al. [39]. Prior work has proposed different wave semantics [9,10,38-40]. OmniUnpack [13] improves unpacking performance by using page permissions and waiting to dump unpacked content until the program invokes a dangerous system call. Guo et al. [41] study the packer problem and propose heuristics to detect the OEP. Their unpacker called Justin enforces the invariant that no code page can be executed without being scanned first. Justin makes two assumptions: (1) the address space layout of the program embedded within a packed binary after it is unpacked is the same as that if the program is directly loaded into memory, and (2) the unpacker in a packed binary completely unpacks the embedded program before transferring control to it. However, Ugarte et al. [9] has shown that many packers do not comply to these assumption.

Ugarte et al. [9] propose a taxonomy of packer complexity and perform a longitudinal study of custom and off-the-shelf packers. They find that multiple assumptions of prior unpacking approaches are violated, e.g., 14\% packed malware samples do not have a single point where all unpacked code is available in memory. They also show that current unpackers may rely on a number of assumptions that may not reflect real 
malware such as that all code will be available at the end of the execution, that the unpacking process is not interleaved with the execution of the original code, or that only one process is involved in the unpacking process.

Bonfante et al. [10] handle this issue by taking a memory snapshot whenever a new wave is detected. They refine the wave semantics by Guizani et al. [39] using a global execution level and introduces a disassembly approach that handles overlapping code. In this work we use the unpacking approach proposed by Bonfante et al. [10]. Rambo [11] combines unpacking with multi-path symbolic execution to handle packers that may only unpack code that needs to be executed. While such packers exist, e.g., Armadillo has an option (disabled by default) to perform page-level unpacking, previous work that examined the landscape of packed malware [9] was not able to find any such real malware. Thus, they evaluate their approach on two synthetic samples. Currently, our approach does not support such packers and we leave the combination with multi-path exploration to future work.

Most of the packers remove original import address table (IAT) that contains external API functions addresses. A packer rebuilds original IAT before transferring control to the original binary code so that the API calls are resolved successfully. BinUnpack [42] exploits this fact and uses Kernel-level DLL hijacking to monitor API calls. It takes a memory snapshot at API call which differs from the IAT of packed executable, i.e., an indication that the original IAT has been built, and thus, original code has been unpacked. BinUnpack provides many orders of magnitude performance boost than write-then-execute approach. Currently, we use write-then-execute approach for malware unpacking. In the future, we would like to experiment with BinUnpack's unpacking approach.

\subsection{Disassembly}

Much work has addressed the challenge of disassembling binary code [43-50], which in general is an undecidable problem [51]. Most related are approaches that address disassembly of malicious code [43-45]. A challenging step during disassembly is identifying the start and end of functions, which can be performed using a machine learning classifier [52,53]. In this work we use ByteWeight [52] for function identification and leverage instruction and function addresses observed during program execution to improve the disassembly [54].

ByteWeight [52] trains a weighted prefix tree (also trie) on the byte sequences or instruction sequences of the function's prologue. In the classification phase, ByteWeight finds a path in the trie for the given sequence of bytes or instructions. A threshold is applied on the cumulative weights of the path to mark the given sequence as function starting address. ByteWeight's training phase is computationally expensive as it takes 587 hours for training on 2,064 binaries. Eui et al. [53] develop a neural network model that automatically learns distinguishing features from the training data and detects function boundaries. Their approach improves on ByteWeight both in training 
overhead and function detection accuracy. However, both the approaches need to train their models for every new compiler and compilation options. Dennis et al. propose Nucleus [55], a tool that identifies function boundaries regardless of the compilers and compilation options.

Our approach collects unpacked original binary code in the form of memory dumps, and thus, our unpacking and disassembly module needs to detect functions from the memory dumps. Unfortunately, both ByteWeight and Eui et al.'s approach only support regular binaries. Nucleus supports memory dump but only for x86-64 architecture. Thus, we prefer to use ByteWeight and we have extended ByteWeight's functionality so that it can be applied on memory dumps.

Binary diffing. Prior work proposes graph-theoretic approaches for comparing two program executables $[26,56,57]$. Such techniques have been implemented in popular IDA binary diffing plugins such as BinDiff [58] and Diaphora [59]. However, those tools cannot handle packed code. Our unpacking and disassembly modules enable using BinDiff and Diaphora for diffing two packed executables. Furthermore, pairwise diffing requires $O\left(n^{2}\right)$ comparisons for $n$ samples, which does not scale to the lineage problem. Instead, we use function hashes to identify in $O(1)$ the same function across samples and program hashes to find in $O(1)$ all samples that correspond to the same version. 


\section{A Systematization of Binary Code Similarity}

In this chapter, we perform a first systematic study on binary code similarity. We systematize four aspects of binary code similarity research: (1) the applications enabled, (2) the approaches employed, (3) how the approaches have been implemented, and (4) the benchmarks and evaluation methodologies. We also present our insights including limitations and future research directions. Our survey shows that the binary code similarity problem has evolved over time. In the last few years, the focus has shifted towards analyzing cross-architecture binary code than mono-architecture. In recent trends, we have seen an increase in the use of machine learning for binary code similarity detection.

\subsection{Introduction}

Over the past 20 years, researchers have proposed many binary code similarity approaches that compare two or more pieces of binary code and compute their similarities. Binary code similarity approaches performs a fine-grain comparison on the disassembled binary code $[43,45-50]$. This enables many real world applications. For instance, open-source software license violation in commercial-of-the-shelf (COTS) binaries can be detected by comparing binary code of COTS binary and open-source program $[60,61]$.

Computing similarities of pieces of binary code is a challenging tasks. The main challenge is the fact that a source code may be compiled using different compilers (e.g., GCC, ICC), optimizations (e.g., O1, O2), operating systems (e.g., Windows, Linux), and CPU architectures (e.g., x86, MIPS). This results in varying binary code for the same source code which adds complexity in computing similarities among pieces of binary code. For instance, CPU architectures vary in instruction sets, and thus, the binary code for different architectures also vary in syntax. In addition, code transformations can be applied on the source code and binary code to further transform the binary code. 
In this chapter, we perform a first systematic survey on binary code similarity research. We begin with a discussion on the origin of binary code similarity and how the problem of binary code similarity has evolved over the past two decades. Then, we systematize four aspects of binary code similarity: (1) the top applications and their relevance to binary code similarity, (2) approaches used for computing similarity among pieces of binary code, (3) how the approaches have been implemented, and (4) benchmarks and evaluation methodologies.

Binary code similarity has a wide list of real-world applications such as bug and vulnerability search [62-75], malware clustering [76-79], malware detection [80-82], malware lineage $[15,31,83]$, porting version information $[26,84,85]$, security patch analysis [26, 56, 69, 85-88], software theft [61].

We have studied more than hundred research articles published in various system security and software engineering conferences, and selected 59 of those which we deem as most relevant to binary code similarity. Our survey shows that approaches use diverse techniques to compare binary code. We group approaches into different categories that capture different dimensions of binary code comparison. The categories include: comparison type, comparison granularity, syntactical (e.g., instruction alignment), structural (e.g., subgraph isomorphism), semantics (e.g., input-output pairs), feature-based, machine learning (e.g., neural networks), hashing (e.g., locality sensitive hashing), type of the analysis (static and dynamic analysis). Our survey reveals that the problem of binary code similarity has evolved over time from a binary code diffing to binary code search of pieces of binary code. In the last few years, the focus has become more on comparing cross-architecture binary code than mono-architecture. And, in recent trends, we have seen an increase in the use approaches that learns the semantics of pieces of binary code automatically.

Other than approaches, we systematize implementation and evaluation of binary code similarity works. For implementation, we systematize the static and dynamic framework used, programming language used, distributed analysis performed, targeted CPU architectures, supported operating systems, and if the approaches are released as open-source and free binaries. For evaluation, we systematize benchmarks, compilers and their optimizations, approach robustness, accuracy, performance, and crosscomparison. Our survey reveals that IDA is the most popular disassembler, and major chunk of approaches (33/59) are mono-architecture and 14 perform cross-architecture binary code similarity. Benchmarks contain both benign and malicious binary programs, and we observe and increasing trend in evaluating the robustness of the approaches against cross-compiler (e.g., GCC vs Clang), cross-optimizations (e.g., O1 vs O3), and cross-architecture (e.g., x86 vs ARM) binary code.

To the best of our knowledge, this work is a first study to conduct a survey on binary code similarity. However, a couple of works $[89,90]$ have surveyed distance and similarity metrics for binary feature vector or numerical feature vector representation. On the contrary, we focus on reviewing approaches that perform binary code similarity. Binary code similarity approaches may utilize similarity metric when binary code is abstracted as feature vectors which we have discussed in Section Section 3.6.6. 


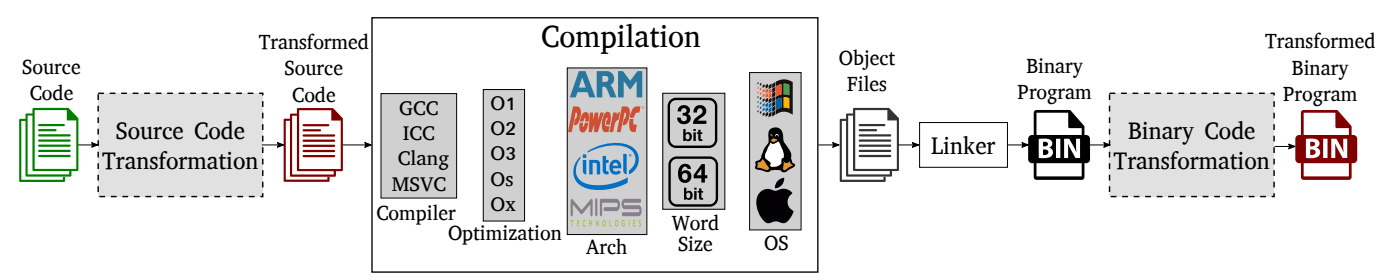

Figure 3.1: The extended compilation process. Dotted boxes are optional code transformations typically used for obfuscation. For a given source code, changing any of the grey boxes may produce a different binary program.

\subsection{Overview}

In this section, we first provide background on the compilation process (Section 3.2.1). Then, we present an overview of the binary code similarity problem (Section 3.2.2).

\subsubsection{Compilation Process}

Binary code refers to the machine code that is produced by the compilation process and that can be run directly by a CPU. The standard compilation process takes as input the source code files of a program. It compiles them with a chosen compiler and optimization level and for a specific platform (defined by the architecture, word size, and OS) producing object files. Those object files are then linked into a binary program, either a stand-alone executable or a library.

Binary code similarity approaches typically deal with an extended compilation process, illustrated in Figure 3.1, which adds two optional steps to the standard compilation process: source code and binary code transformations. Both types of transformations are typically semantics-preserving (i.e., do not change the program functionality) and are most commonly used for obfuscation, i.e., to hamper reverse-engineering of the distributed binary programs. Source code transformations happen pre-compilation. Thus, their input and output are both source code. They can be applied regardless of the target platform, but may be specific to the programming language used to write the program. On the other hand, binary code transformations happen post-compilation. Thus, their input and output are binary code. They are independent of the programming language used, but may be specific to the target platform.

Obfuscation is a fundamental step in malware, but can also be applied to benign programs, e.g., to protect their intellectual property. There exist off-the-shelf obfuscation tools that use source code transformations (e.g., Tigress [91]), as well as binary code transformations (e.g., packers). Packing is a binary code transformation widely used by malware. Once a new version of a malware family is ready, the malware authors pack the resulting executable to hide its functionality and thus bypass detection by commercial malware detectors. The packing process takes as input an executable and produces another executable with the same functionality, but with the original code hidden (e.g., encrypted as data and unpacked at runtime). The packing process is typi- 
cally applied many times to the same input executable, creating polymorphic variants of exactly the same source code, which look different to malware detectors. Nowadays, the majority of malware is packed and malware often uses custom packers for which off-the-shelf unpackers are not available [9].

A main challenge in binary code similarity is that the compilation process can produce different binary code representations for the same source code. An author can modify any of the grey boxes in Figure 3.1 to produce a different, but semanticallyequivalent, binary program from the same source code. Some of these modifications may be due to the standard compilation process. For example, to improve program efficiency an author may vary the compiler's optimization level, or change the compiler altogether. Both changes will transform the produced binary code, despite the source code remaining unchanged. An author may also change the target platform to obtain a version of the program suitable for a different architecture. In this case, the produced binary code may radically differ if the new target architecture uses a different instruction set. An author may also deliberately apply obfuscation transformations to produce polymorphic variants of the same source code. The produced variants will typically have the same functionality defined by the original source code. A desirable goal for binary code similarity approaches is that they are able to identify the similarity of binary code that corresponds to the same source code having undergone different transformations. The robustness of a binary code similarity approach captures the compilation and obfuscation transformations that it can handle, i.e., the transformations despite which it can still detect similarity.

\subsubsection{Binary Code Similarity Overview}

Binary code similarity approaches compare pieces of binary code. The three main characteristics of binary code similarity approaches are: (1) the type of the comparison (identical, similar, equivalent), (2) the granularity of the pieces of binary code being compared (e.g., instructions, basic blocks, functions), and (3) the number of input pieces being compared (one-to-one, one-to-many, many-to-many). We detail these three characteristics next. For simplicity, we describe the comparison type and comparison granularity for two inputs and then generalize to multiple inputs.

Comparison type. Two (or more) pieces of binary code are identical if they have the same syntax, i.e., the same representation. The binary code can be represented in different ways such as an hexadecimal string of raw bytes, a sequence of disassembled instructions, or a control-flow graph. Determining if several pieces of binary code are identical is a Boolean decision (either they are identical or not) that it is easy to check: simply apply a cryptographic hash (e.g., MD5, SHA1, SHA256, SHA512) to the contents of each piece. If the hash is the same, the pieces are identical. However, such straightforward approach fails to detect similarity in many cases. For example, compiling the same source code twice, using the same compilation parameters (i.e., same compiler version, same optimization level, same target platform) produces two 


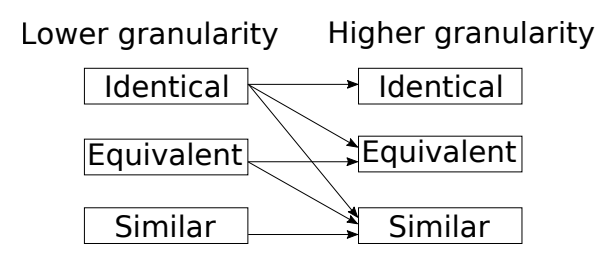

Figure 3.2: Similarity at a finer granularity can be used to infer a different type of similarity at a coarser granularity.

executables with different file hash. This happens because the executable may include metadata that differs in both compilations such as the compilation date, which is automatically computed and included into the header of the generated executable.

Two pieces of binary code are equivalent if they have the same semantics, i.e., if they offer exactly the same functionality. Equivalence does not care about the syntax of the binary code. For example, the same source code compiled for two different target architectures should produce equivalent executables, whose syntax may be completely different if the architectures use different instruction sets. At the instruction granularity, mov REG, $\$ 0$ and xor REG, REG are semantically equivalent instructions because both set the value of register REG to zero. Determining binary code equivalence is a very expensive process that can only be performed for small pieces of binary code. In fact, proving that two programs are functionally equivalent is an undecidable problem that reduces to solving the halting problem [92].

Two pieces of binary code can be considered similar if their syntax or their semantics are similar. Semantic similarity is used, for example, in malware clustering, where malicious executables with similar behaviors are grouped into the same family cluster [93]. Behaviors represent the code semantics, often captured by the interaction between the program and its environment through OS APIs or system calls. Other applications use syntactic similarity. For example, clone detection considers that a target piece of binary code is a clone of some source binary code if their syntax are similar. Syntactic similarity approaches are in general cheaper to compute, but they are sensitive to changes in the binary code, e.g., register reallocation, instruction reordering, replacing instructions with semantically equivalent ones. The robustness of a syntactic similarity approach is defined by the transformations that it can handle. On the other hand, semantic similarity is robust against semantics-preserving transformations, but it is very expensive and complex to compute for large pieces of binary code.

Comparison granularity. Binary code similarity approaches can be applied at different granularities. Common granularities are instructions; basic blocks; functions; and whole programs. To perform a comparison at a coarser granularity, some approaches use a different comparison at a finer granularity, and then combine the finer granularity results. For example, to compare whether two programs are similar, an approach could determine the fraction of identical functions between both programs. Thus, we differentiate between the input granularity, i.e., the granularity of the input pieces of binary code that the approach compares, and the approach granularities, i.e., the granularities 
of the different comparisons in the approach.

Applying a specific comparison at a finer granularity may restrict the type of comparison that can be performed at a coarser granularity, as illustrated in Figure 3.2. The figure shows that computing whether two pieces of binary code are identical at a finer granularity (e.g., basic block) can be used to compute that the coarser granularity pieces that encompass them (e.g., their functions) are identical, equivalent, or similar. However, similarity at a finer granularity cannot be used to infer that the coarser granularity code is equivalent or identical. For example, when comparing two functions, just because all their basic blocks are similar, it cannot be concluded that the functions are identical or equivalent. On the other hand, similarity is the most general type of comparison and any finer granularity comparison type can be used to infer it.

Number of inputs. Binary code similarity approaches can compare two or more pieces of binary code. Those that compare more than two pieces can further be split into comparing one piece to the rest or comparing each piece to all other pieces. Thus, we identify three types of approaches based on the number of inputs and how they are compared: one-to-one $(O O)$, one-to-many $(O M)$, and many-to-many $(M M)$. The source of the input pieces is application-dependent. They may come from the same program version (e.g., two functions of the same executable), from two versions of the same program, and from two different programs.

One-to-one approaches compare an original piece of binary code (also called old, source, plaintiff, or reference) to a target piece of binary code (also called new, patched, or upgrade). Most $O O$ approaches perfom binary code diffing, i.e., they diff two consecutive versions of the same program to identify what was added, removed, or modified in the target (patched) version. The granularity of binary code diffing is most often functions and the diffing tries to obtain a mapping between a function in the original program version and another function in the target program version. Added functions are original functions that cannot be mapped to a target function; removed functions are target functions that cannot be mapped to an original function; and modified functions are mapped functions that are not identical.

One-to-many approaches compare a query piece of binary code to many target pieces of binary code. Most $O M$ approaches perform binary code search, i.e., they search if the query piece is similar to any of the target pieces. The target pieces may come from multiple versions of the same program (different than the version the query piece comes from), from different programs compiled for the same architecture, or from programs compiled for different architectures.

In contrast to $O O$ and $O M$ approaches, many-to-many approaches do not distinguish between source and target pieces. All input pieces are considered equal and compared against each other. These approaches typically perform binary clustering, i.e., they output groups of similar pieces of binary code called clusters. 
Table 3.1: Comparison among binary code similarity approaches. For boolean columns $\checkmark$ means supported and $\boldsymbol{X}$ unsupported. Input Comparison can be one-to-one (OO), one-to-many (OM), or many-to-many (MM). Input Granularity and Approach Granularities can be instruction (I), basic block (B), function (F), or program (P). Approach Comparison can be Similar (S), Identical (I), or Equivalent (E). Structural similarity can use CFG (C), ICFG (I), Callgraph (G), and other custom graphs (O). Machine learning can be Supervised (S) or Unsupervised (U). In Normalization, $\boldsymbol{X}$ means no normalization, $\diamond$ operand removal, $\bullet$ operand normalization, $\circ$ mnemonic normalization, and $\star$ code elimination.

\begin{tabular}{|c|c|c|c|c|c|c|c|c|c|c|c|c|c|c|c|c|c|}
\hline \multirow[b]{2}{*}{ Approach } & \multirow[b]{2}{*}{ Year } & \multirow[b]{2}{*}{ Venue } & \multicolumn{15}{|c|}{ Approach Characteristics } \\
\hline & & & 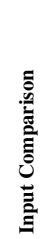 & 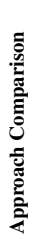 & 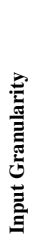 & 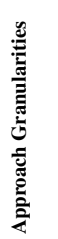 & 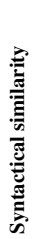 & 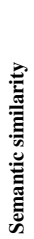 & 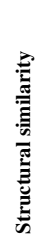 & 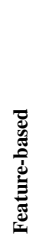 & 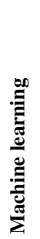 & 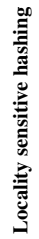 & 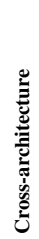 & 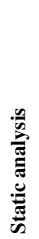 & 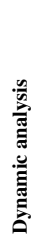 & 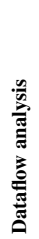 & 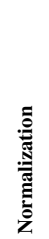 \\
\hline EXEDIFF [94] & 1999 & WCSSS & $\mathrm{OO}$ & I & $P$ & I & $\checkmark$ & $x$ & $x$ & $x$ & $x$ & $x$ & $x$ & $\checkmark$ & $x$ & $x$ & $\bullet$ \\
\hline BMAT [84] & 1999 & FDO2 & OO & S,I & $\mathrm{P}$ & $\mathrm{F}, \mathrm{B}$ & $\checkmark$ & $x$ & $\mathrm{C}$ & $x$ & $x$ & $x$ & $x$ & $\checkmark$ & $x$ & $x$ & $\diamond \bullet$ \\
\hline F2004 [26] & 2004 & DIMVA & $\mathrm{OO}$ & $\mathrm{s}$ & $\mathrm{P}$ & $\mathrm{F}$ & $x$ & $x$ & $\mathrm{C}, \mathrm{G}$ & $\checkmark$ & $x$ & $x$ & $x$ & $\checkmark$ & $x$ & $x$ & $x$ \\
\hline DR2005 [85] & 2005 & SSTIC & $\mathrm{OO}$ & S,I & $\mathrm{P}$ & $\mathrm{F}, \mathrm{B}, \mathrm{I}$ & $\checkmark$ & $x$ & $\mathrm{C}, \mathrm{G}$ & $\checkmark$ & $x$ & $x$ & $x$ & $\checkmark$ & $x$ & $x$ & $\diamond \bullet$ \\
\hline KKMRV2005 [80] & 2005 & RAID & MM & $\mathrm{s}$ & $\mathrm{P}$ & $\mathrm{B}^{*}$ & $x$ & $\checkmark$ & I & $x$ & $x$ & $x$ & $x$ & $\checkmark$ & $x$ & $x$ & $\diamond \bullet$ \\
\hline BMM2006 [81] & 2006 & DIMVA & $\mathrm{OO}$ & $\mathrm{s}$ & $\mathrm{P}$ & $\mathrm{B}^{*}$ & $x$ & $\checkmark$ & I & $x$ & $x$ & $x$ & $x$ & $\checkmark$ & $x$ & $\checkmark$ & $\diamond \bullet$ \\
\hline BinHUNT [56] & 2008 & ICISC & $\mathrm{OO}$ & S,E & $\mathrm{P}$ & $\mathrm{F}, \mathrm{B}$ & $x$ & $\checkmark$ & $\mathrm{C}, \mathrm{G}$ & $x$ & $x$ & $x$ & $x$ & $\checkmark$ & $x$ & $\checkmark$ & $x$ \\
\hline SWPQS 2006 [95] & 2009 & ISSTA & MM & S,I & $I^{*}$ & $\mathrm{I}^{*}$ & $\checkmark$ & $x$ & $x$ & $\checkmark$ & $x$ & $\checkmark$ & $x$ & $\checkmark$ & $x$ & $x$ & $\bullet$ \\
\hline SMIt [76] & 2009 & CCS & $\mathrm{OM}$ & S,I & $\mathrm{P}$ & $\mathrm{F}$ & $\checkmark$ & $x$ & G & $\checkmark$ & $x$ & $x$ & $x$ & $\checkmark$ & $x$ & $x$ & $x$ \\
\hline IDEA [96] & 2010 & ESSoS & MM & $\mathrm{s}$ & $\mathrm{P}$ & I* $^{*}$ & $\checkmark$ & $x$ & $x$ & $\checkmark$ & $x$ & $x$ & $x$ & $\checkmark$ & $x$ & $x$ & • \\
\hline BITSHRED [77] & 2011 & CCS & MM & $\mathrm{s}$ & $\mathrm{P}$ & $I^{*}$ & $\checkmark$ & $x$ & $x$ & $\checkmark$ & $\mathrm{U}$ & $x$ & $x$ & $\checkmark$ & $\checkmark$ & $x$ & $x$ \\
\hline MBC [97] & 2012 & RACS & MM & $\mathrm{s}$ & $\mathrm{P}$ & B & $\checkmark$ & $x$ & $x$ & $\checkmark$ & $x$ & $x$ & $x$ & $\checkmark$ & $x$ & $x$ & $\bullet$ \\
\hline IBINHUNT [98] & 2012 & ICISC & OO & S,E & $\mathrm{P}$ & B & $x$ & $\checkmark$ & I & $x$ & $x$ & $x$ & $x$ & $\checkmark$ & $\checkmark$ & $\checkmark$ & $x$ \\
\hline BEAGLE [31] & 2012 & ACSAC & MM & $\mathrm{s}$ & $\mathrm{P}$ & $\mathrm{B}^{*}$ & $x$ & $\checkmark$ & $\mathrm{C}$ & $x$ & $x$ & $x$ & $x$ & $\checkmark$ & $\checkmark$ & $x$ & • \\
\hline BINHASH [99] & 2012 & ICMLA & $\mathrm{MM}$ & $\mathrm{E}$ & $\mathrm{F}$ & B & $x$ & $\checkmark$ & $x$ & $\checkmark$ & $\mathrm{U}$ & $\checkmark$ & $x$ & $\checkmark$ & $x$ & $\checkmark$ & - \\
\hline BinJuice [92] & 2013 & PPREW & $\mathrm{OO}$ & S,E & $\mathrm{P}$ & $\mathrm{F}, \mathrm{B}$ & $x$ & $\checkmark$ & $x$ & $x$ & $x$ & $x$ & $x$ & $\checkmark$ & $x$ & $\checkmark$ & $x$ \\
\hline BINSLAYER [57] & 2013 & PPREW & $\mathrm{OO}$ & $\mathrm{s}$ & $\mathrm{P}$ & $\mathrm{F}, \mathrm{B}$ & $x$ & $x$ & $\mathrm{C}, \mathrm{G}$ & $x$ & $x$ & $x$ & $x$ & $\checkmark$ & $x$ & $x$ & $\diamond \bullet$ \\
\hline RENDEZvous [100] & 2013 & MSR & OM & $\mathrm{s}$ & $\mathrm{F}$ & $\mathrm{F}$ & $\checkmark$ & $x$ & $x$ & $x$ & $x$ & $x$ & $x$ & $\checkmark$ & $x$ & $x$ & $\diamond \bullet$ \\
\hline MutantX-S [78] & 2013 & Usenix ATC & MM & $\mathrm{s}$ & $\mathrm{P}$ & $\mathrm{I}^{*}$ & $\checkmark$ & $x$ & $x$ & $\checkmark$ & $\mathrm{U}$ & $x$ & $x$ & $\checkmark$ & $x$ & $x$ & $\diamond \bullet$ \\
\hline EXPOSÉ [60] & 2013 & COMPSAC & $\mathrm{OM}$ & S,E & $\mathrm{P}$ & $\mathrm{F}, \mathrm{I}^{*}$ & $\checkmark$ & $\checkmark$ & $x$ & $\checkmark$ & $x$ & $x$ & $x$ & $\checkmark$ & $x$ & $\checkmark$ & • \\
\hline ILINE [15] & 2013 & USENIX Sec & MM & $\mathrm{s}$ & $\mathrm{P}$ & $\mathrm{B}, \mathrm{I}^{*}$ & $\checkmark$ & $x$ & $x$ & $\checkmark$ & $\mathrm{U}$ & $x$ & $x$ & $\checkmark$ & $\checkmark$ & $x$ & $\bullet \circ \star$ \\
\hline LKI2013 [101] & 2013 & RACS & $\mathrm{OO}$ & $\mathrm{s}$ & $\mathrm{P}$ & $\mathrm{F}, \mathrm{I}^{*}$ & $x$ & $x$ & $\mathrm{C}, \mathrm{G}$ & $\checkmark$ & $x$ & $x$ & $x$ & $\checkmark$ & $x$ & $x$ & $\diamond \bullet$ \\
\hline TRACY [62] & 2014 & PLDI & $\mathrm{OM}$ & S,E & $\mathrm{F}$ & $I^{*}$ & $\checkmark$ & $\checkmark$ & $x$ & $x$ & $x$ & $x$ & $x$ & $\checkmark$ & $x$ & $\checkmark$ & $\star$ \\
\hline BinClone [102] & 2014 & SERE & MM & S,I & $I^{*}$ & I* & $\checkmark$ & $x$ & $x$ & $\checkmark$ & $x$ & $x$ & $x$ & $\checkmark$ & $x$ & $x$ & - \\
\hline RMKNHLLP2014 [103] & 2014 & DIMVA & MM & $\mathrm{s}$ & $\mathrm{F}^{*}$ & $\mathrm{~F}$ & $x$ & $x$ & $x$ & $\checkmark$ & $\mathrm{U}$ & $x$ & $x$ & $\checkmark$ & $x$ & $\checkmark$ & $x$ \\
\hline CXZ2014 [82] & 2014 & TDSC & $\mathrm{OM}$ & $\mathrm{s}$ & $\mathrm{P}$ & $\mathrm{F}$ & $x$ & $x$ & $\mathrm{C}$ & $\checkmark$ & $x$ & $x$ & $x$ & $\checkmark$ & $x$ & $x$ & $x$ \\
\hline BLEX [104] & 2014 & USENIX Sec & $\mathrm{OO}$ & $\mathrm{s}$ & $\mathrm{F}$ & $\mathrm{F}$ & $x$ & $\checkmark$ & $x$ & $\checkmark$ & $x$ & $x$ & $x$ & $\checkmark$ & $\checkmark$ & $x$ & $x$ \\
\hline $\operatorname{CoP}[61,105]$ & 2014 & ESEC/FSE & $\mathrm{OO}$ & S,E & $\mathrm{P}$ & $\mathrm{F}, \mathrm{B}$ & $x$ & $\checkmark$ & $\mathrm{C}$ & $x$ & $x$ & $x$ & $x$ & $\checkmark$ & $x$ & $\checkmark$ & $x$ \\
\hline TEDEM [63] & 2014 & ACSAC & $\mathrm{OM}$ & $\mathrm{s}$ & $\mathrm{B}^{*}$ & B & $x$ & $\checkmark$ & $\mathrm{C}$ & $x$ & $x$ & $x$ & $x$ & $\checkmark$ & $x$ & $x$ & $x$ \\
\hline Sigma [106] & 2015 & DFRWS & OO & $\mathrm{s}$ & $\mathrm{F}$ & $\mathrm{F}$ & $x$ & $x$ & $\mathrm{O}$ & $x$ & $x$ & $x$ & $x$ & $\checkmark$ & $x$ & $x$ & $\diamond \bullet$ \\
\hline MXW2015 [83] & 2015 & IFIP SEC & $\mathrm{OO}$ & $\mathrm{E}$ & $\mathrm{P}$ & B & $x$ & $\checkmark$ & I & $x$ & $x$ & $x$ & $x$ & $\checkmark$ & $\checkmark$ & $\checkmark$ & $\bullet \star \star$ \\
\hline Multi-MH [64] & 2015 & $S \& P$ & OM & $\mathrm{s}$ & B* & B & $x$ & $\checkmark$ & $\mathrm{C}$ & $x$ & $x$ & $\checkmark$ & $\checkmark$ & $\checkmark$ & $x$ & $\checkmark$ & $x$ \\
\hline QSM2015 [107] & 2015 & SANER & $\mathrm{OO}$ & I & $\mathrm{F}$ & I* $^{*}$ & $x$ & $x$ & $\mathrm{O}$ & $x$ & $x$ & $x$ & $x$ & $\checkmark$ & $x$ & $\checkmark$ & $\bullet \star \star$ \\
\hline DISCOVRE [65] & 2016 & NDSS & OM & $\mathrm{s}$ & $\mathrm{F}$ & B & $x$ & $x$ & $\mathrm{C}$ & $\checkmark$ & $x$ & $x$ & $\checkmark$ & $\checkmark$ & $x$ & $x$ & $x$ \\
\hline MOCKINGBIRD [86] & 2016 & SANER & OM & $\mathrm{s}$ & $\mathrm{F}$ & $\mathrm{F}$ & $x$ & $\checkmark$ & $x$ & $x$ & $x$ & $x$ & $\checkmark$ & $x$ & $\checkmark$ & $x$ & $x$ \\
\hline Esh [66] & 2016 & PLDI & $\mathrm{OM}$ & E & $\mathrm{F}$ & I* $^{*}$ & $x$ & $\checkmark$ & $x$ & $x$ & $x$ & $x$ & $x$ & $\checkmark$ & $x$ & $\checkmark$ & $x$ \\
\hline ТPM [108] & 2016 & TrustCom & $\mathrm{OO}$ & $\mathrm{s}$ & $\mathrm{P}$ & $\mathrm{F}$ & $x$ & $x$ & $x$ & $\checkmark$ & $x$ & $x$ & $x$ & $\checkmark$ & $x$ & $x$ & $x$ \\
\hline BINDNN [109] & 2016 & SecureComm & OM & $\mathrm{s}$ & $\mathrm{F}$ & $\mathrm{F}$ & $x$ & $x$ & $x$ & $x$ & $\mathrm{~S}$ & $x$ & $\checkmark$ & $\checkmark$ & $x$ & $x$ & • \\
\hline Genius [67] & 2016 & CCS & $\mathrm{OM}$ & $\mathrm{s}$ & $\mathrm{F}$ & B & $x$ & $x$ & $\mathrm{C}$ & $\checkmark$ & $\mathrm{U}$ & $\checkmark$ & $\checkmark$ & $\checkmark$ & $x$ & $x$ & $x$ \\
\hline BINGo [68] & 2016 & FSE & $\mathrm{OM}$ & $\mathrm{s}$ & $\mathrm{F}$ & $\mathrm{B}^{*}, \mathrm{I}$ & $x$ & $\checkmark$ & $x$ & $x$ & $x$ & $x$ & $\checkmark$ & $\checkmark$ & $x$ & $\checkmark$ & $\star$ \\
\hline KLKI2016 [79] & 2016 & JSCOMPUT & $\mathrm{OO}$ & $\mathrm{s}$ & $\mathrm{P}$ & $\mathrm{F}$ & $x$ & $x$ & $\mathrm{G}$ & $\checkmark$ & $x$ & $x$ & $x$ & $\checkmark$ & $\checkmark$ & $x$ & $x$ \\
\hline KAM1N0 [110] & 2016 & SIGKDD & $\mathrm{OM}$ & $\mathrm{S}$ & $\mathrm{B}^{*}$ & B & $\checkmark$ & $x$ & $\mathrm{C}$ & $\checkmark$ & $x$ & $\checkmark$ & $x$ & $\checkmark$ & $x$ & $x$ & - \\
\hline BINSEQUENCE [69] & 2017 & ASIACCS & OM & $\mathrm{S}$ & $\mathrm{F}$ & $\mathrm{B}, \mathrm{I}$ & $\checkmark$ & $x$ & $\mathrm{C}$ & $x$ & $x$ & $\checkmark$ & $x$ & $\checkmark$ & $x$ & $x$ & - \\
\hline ХМАТCH [70] & 2017 & ASIACCS & OM & $\mathrm{s}$ & $\mathrm{F}$ & $\mathrm{I}^{*}$ & $x$ & $\checkmark$ & $x$ & $x$ & $x$ & $x$ & $\checkmark$ & $\checkmark$ & $x$ & $\checkmark$ & $x$ \\
\hline CACOMPARE [111] & 2017 & ICPC & OM & $\mathrm{s}$ & $\mathrm{F}$ & $\mathrm{F}$ & $x$ & $\checkmark$ & $x$ & $x$ & $x$ & $\checkmark$ & $\checkmark$ & $\checkmark$ & $x$ & $x$ & $x$ \\
\hline SPain [87] & 2017 & ICSE & $\mathrm{OO}$ & S,I & $\mathrm{P}$ & $\mathrm{F}, \mathrm{B}$ & $\checkmark$ & $\checkmark$ & $x$ & $x$ & $x$ & $x$ & $x$ & $\checkmark$ & $x$ & $\checkmark$ & $\bullet$ \\
\hline BINSIGN [112] & 2017 & IFIP SE & $\mathrm{OM}$ & S & $\mathrm{F}$ & $\mathrm{F}$ & $x$ & $x$ & $x$ & $\checkmark$ & $x$ & $\checkmark$ & $x$ & $\checkmark$ & $x$ & $x$ & • \\
\hline GitZ [71] & 2017 & PLDI & $\mathrm{OM}$ & E & $\mathrm{F}$ & I* $^{*}$ & $x$ & $\checkmark$ & $x$ & $x$ & $x$ & $x$ & $\checkmark$ & $\checkmark$ & $x$ & $x$ & $x$ \\
\hline BINSHAPE [113] & 2017 & DIMVA & $\mathrm{OM}$ & $\mathrm{s}$ & $\mathrm{F}$ & $\mathrm{F}$ & $x$ & $x$ & $x$ & $\checkmark$ & $x$ & $\checkmark$ & $x$ & $\checkmark$ & $x$ & $x$ & $\bullet$ \\
\hline BINSIM [114] & 2017 & USENIX Sec & $\mathrm{OO}$ & $\mathrm{s}$ & $\mathrm{T}$ & I* $^{*}$ & $x$ & $\checkmark$ & $x$ & $x$ & $x$ & $x$ & $x$ & $x$ & $\checkmark$ & $\checkmark$ & $x$ \\
\hline KS 2017 [88] & 2017 & ASE & $\mathrm{OM}$ & $\mathrm{s}$ & $\mathrm{T}$ & I* $^{*}$ & $x$ & $\checkmark$ & $x$ & $\checkmark$ & $x$ & $x$ & $x$ & $x$ & $\checkmark$ & $x$ & $x$ \\
\hline GEMINI [72] & 2017 & CCS & OM & $\mathrm{s}$ & $\mathrm{F}$ & $\mathrm{F}$ & $x$ & $x$ & $\mathrm{C}$ & $\checkmark$ & $\mathrm{s}$ & $\checkmark$ & $\checkmark$ & $\checkmark$ & $x$ & $x$ & $x$ \\
\hline Fossil [115] & 2018 & TOPS & $\mathrm{OM}$ & $\mathrm{s}$ & $\mathrm{F}$ & $\mathrm{F}, \mathrm{B}^{*}$ & $x$ & $\checkmark$ & $\mathrm{C}$ & $\checkmark$ & $x$ & $x$ & $x$ & $\checkmark$ & $x$ & $x$ & - \\
\hline FIRMUP [73] & 2018 & ASPLOS & $\mathrm{OM}$ & $\mathrm{E}$ & $\mathrm{F}$ & $\mathrm{I}^{*}$ & $x$ & $\checkmark$ & $x$ & $x$ & $x$ & $x$ & $\checkmark$ & $\checkmark$ & $x$ & $x$ & $\bullet$ \\
\hline BINARM [74] & 2018 & DIMVA & OM & $\mathrm{s}$ & $\mathrm{F}$ & $\mathrm{F}$ & $x$ & $x$ & $\mathrm{C}$ & $\checkmark$ & $x$ & $x$ & $x$ & $\checkmark$ & $x$ & $x$ & - \\
\hline$\alpha \operatorname{DIFF}[75]$ & 2018 & ASE & $\mathrm{OO}$ & $\mathrm{s}$ & $\mathrm{P}$ & $\mathrm{F}$ & $x$ & $x$ & $x$ & $x$ & $\mathrm{~S}$ & $x$ & $\checkmark$ & $\checkmark$ & $x$ & $x$ & $x$ \\
\hline RLZ2019 [116] & 2019 & BAR & $\mathrm{OM}$ & $\mathrm{s}$ & B & B & $x$ & $x$ & $x$ & $x$ & $\mathrm{~S}$ & $x$ & $\checkmark$ & $\checkmark$ & $x$ & $x$ & $\bullet$ \\
\hline INNEREYE [117] & 2019 & NDSS & $\mathrm{OM}$ & $\mathrm{s}$ & $\mathrm{B}^{*}$ & B & $x$ & $x$ & $x$ & $x$ & $\mathrm{~S}$ & $\checkmark$ & $\checkmark$ & $\checkmark$ & $x$ & $x$ & - \\
\hline ASM2VEC [118] & 2019 & $S \& P$ & $\mathrm{OM}$ & $\mathrm{s}$ & $\mathrm{F}$ & $I^{*}$ & $x$ & $x$ & $x$ & $x$ & $\mathrm{~S}$ & $x$ & $x$ & $\checkmark$ & $x$ & $x$ & $x$ \\
\hline
\end{tabular}




\subsection{Scope \& Paper Selection}

To keep our survey of the state-of-the-art focused and manageable it is important to define what is, and what is not, within scope. Overall, the main restriction is that we focus on works that compare binary code. This restriction, in turn, introduces the following four constraints:

1. We exclude approaches that require access to the source code, namely sourceto-source (e.g., [119]) and source-to-binary (e.g., [120]) similarity approaches.

2. We exclude approaches that operate on bytecode (e.g., [121, 122]).

3. We exclude behavioral approaches that compare similarity exclusively on the interaction of a program with its environment through system calls or OS API calls (e.g., [123-126]).

4. We exclude approaches that consider binary code as a sequence of raw bytes with no structure such as file hashes (e.g., [127]), fuzzy hashes (e.g., [128, 129]), and signature-based approaches (e.g., $[130,131])$. Approaches need to disassemble raw bytes into instructions to be considered. While we do not include the papers describing byte-level approaches, we do examine the use of some of those techniques (e.g., fuzzy hashing) by the analyzed approaches.

In addition, we introduce the following constraints to keep the scope of the survey manageable:

5. We limit the survey to papers published on peer-reviewed venues and technical reports from academic institutions. Thus, we do not analyze tools, but rather the research works describing their approach (e.g., $[26,85]$ for BINDIFF)

6. We exclude papers that do not propose a new binary code similarity approach or technique, but simply apply off-the-shelf binary code similarity tools as a step towards their goal.

Paper selection. To identify candidate papers, we first systematically examined all papers published in the last 20 years in 14 top venues for computer security and software engineering: IEEE S\&P, ACM CCS, USENIX Security, NDSS, ACSAC, RAID, ESORICS, ASIACCS, DIMVA, ICSE, FSE, ISSTA, ASE, and MSR. Not all relevant binary code similarity approaches have been published in those venues, which is especially true for early approaches. To identify candidate papers in other venues, we extensively queried specialized search engines such as Google Scholar using terms related to binary code similarity and its applications, e.g., code search, binary diffing, bug search. We also carefully examined the references of the candidate papers for any further papers we may have missed. This exploration identified over a hundred candidate 
papers. We then read each candidate paper to determine if they proposed a binary code similarity approach that satisfied the above scope constraints.

In the end, we identified the 59 binary code similarity research works in Table 3.1, whose approaches are analyzed in detail. The first three columns of Table 3.1 capture the name of the approach, the year of publication, and the venue where the work was published. The research works are sorted by publication date creating a timeline of the development of the field. Papers are identified by their system name, if available, otherwise by the initials of each author's last name and the year of publication. The 59 papers have been published in 30 venues. Binary code similarity is quite multidisciplinary; while most papers appear in computer security venues (32 papers in 16 venues), there are works in software engineering (9 papers in 7 venues), systems (5 papers in 3 venues), and machine learning (2 papers in 2 venues). The venues with most binary code similarity papers are: DIMVA (5), CCS (4), USENIX Security (3), and PLDI (3).

\subsection{Binary Code Similarity Evolution}

This section describes the origins of binary code similarity and its evolution over the last 20 years, highlighting some noteworthy approaches.

The origins. The origins of binary code similarity are in the problem of generating a patch (or delta) that captures the differences between two consecutive (or close) versions of the same program. Text diffing tools had existed since the 1970's (the popular UNIX diff was released in 1974) and had been integrated in early source code versioning systems such as SCCS [132] (1975) and RCS [133] (1985). The increasing popularity of low bandwidth communication networks (e.g., wireless) and the limited resources in some devices, raised interest in techniques that would increase efficiency by transmitting a small patch that captured the differences between two versions of a binary, i.e., non-text, file, instead of transmitting the whole file. In 1991, Reichenberger proposed a diffing technique for generating patches between arbitrary binary files without any knowledge about the file structure [134]. The approach worked at the byte-level, instead of the line-level granularity of text diffing, and efficiently identified the byte sequences that should appear in the patch because they only appeared in the updated version and thus were not available in the original file to be patched. Several tools for generating and applying binary patches soon appeared such as RTPATCH [135], BINDIFF95 [136], and XDELTA [137]. Those tools worked at byte-level and could diff any type of file.

The first binary code similarity approaches are from 1999. That year, Baker et al. proposed an approach for compressing differences of executable code and built a prototype diffing tool called EXEDIFF [94], which generated patches for DEC Alpha executables. Their intuition was that many of the changes when diffing two executable versions of the same program represent secondary changes due to the compilation process, as opposed to direct changes in the source code. One example they mentioned is 
register allocation that may change at recompilation. Another example they mentioned is that code added in the newer version would displace parts of the old code, and thus the compilation process would have to adjust pointer values in the displaced code to point to the correct addresses. Their idea was to reconstruct secondary changes at patch time, so that they would not need to be included in the patch, reducing the patch size. EXEDIFF is the earliest approach we have identified that focused on computing similarity between binary code, taking advantage of the code structure by disassembling the raw bytes into instructions.

Also in 1999, Wang et al. presented BMAT [84], a tool that aligned two versions of a Windows DLL library executable to propagate profile information from the older (extensively profiled) version to a newer version, thus reducing the need for reprofiling. Their approach is the first to compare functions and basic blocks (EXEDIFF compared two sequences of instructions). It first matched functions in the two executables and then matched similar blocks within each matched function. It used a hashing technique to compare blocks. The hashing removed relocation information to handle pointer changes, but was order-sensitive.

The first decade. After the initial works of EXEDIFF and BMAT, we only identify 7 binary code similarity approaches in the next decade (2000-2009). However, some of these are highly influential as they extend binary code similarity from purely syntactical to also include semantics; they widen the scope from binary code diffing (OO) to also include binary clustering (MM) and binary code search (OM); and they apply binary code similarity to malware.

In 2004, Thomas Dullien (alias Halvar Flake) proposed a graph-based binary code diffing approach that focused on the structural properties of the code by heuristically constructing a callgraph isomorphism that aligns functions in two versions of the same binary program [26]. This is the first approach to handle instruction reordering inside a function introduced by some compiler optimizations. A followup work [85] extended the approach to also match basic blocks inside matched functions (as done in BMAT) and introduced the Small Primes Product (SPP) hash to identify similar basic blocks despite instruction reordering. These two works are the basis for the popular BINDIFF binary code diffing plugin for the IDA disassembler [58].

In 2005, Kruegel et al. proposed a graph coloring technique to detect polymorphic variants of a malware. This is the first approach that performed semantic similarity and MM comparison. They categorized instructions with similar functionality into 14 semantic classes. Then, they colored the inter-procedural control-flow graph (ICFG) using those classes. The graph coloring is robust against syntactical obfuscations such as junk insertion, instruction reordering, and instruction replacement. In 2008, Gao et al. proposed BINHUNT [56] to identify semantic differences between two versions of the same program. This is the first approach that checks code equivalence. It uses symbolic execution and a constraint solver to check if two basic blocks provide the same functionality.

In 2009, Xin et al. presented SMIT [76], an approach that given a malware sample 
Table 3.2: Applications evaluated in the analyzed binary code similarity research works.

\begin{tabular}{|l|l|}
\hline \multicolumn{1}{|c|}{ Application } & \multicolumn{1}{c|}{ Research works } \\
\hline Bug Search & \multicolumn{1}{c|}{ TRACY [62], TEDEM [63], MULTI-MH [64], DISCOVRE [65], ESH [66], GENIUS [67], BINGo [68] } \\
& BINSEQUENCE [69], XMATCH [70], GITZ [71], GEMINI [72], FIRMUP [73], BINARM [74], $\alpha$ DIFF [75] \\
\hline Malware Clustering & SMIT [76], BITSHRED [77], MUTANTX-S [78], KLKI2016 [79] \\
\hline Malware Detection & KKMRV2005 [80], BMM2006 [81], CXZ2014 [82] \\
\hline Malware Lineage & BEAGLE [31], ILINE [15], MXW2015 [83] \\
\hline Patch Analysis & $\begin{array}{l}\text { F2004 [26], DR2005 [85], BINHUNT [56] } \\
\text { MocKINGBIRD [86], BINSEQUENCE [69], SPAIN [87], KS2017 [88] }\end{array}$ \\
\hline Patch Generation & EXEDIFF [94] \\
\hline Porting information & BMAT [84], F2004 [26], DR2005 [85] \\
\hline Software Theft & CoP [61] \\
\hline
\end{tabular}

finds similar malware in a repository. SMIT is the first OM and binary code search approach. It indexes malware callgraphs in a database and uses graph edit distance to find malware with similar callgraphs.

The last decade. The last decade (2010-2019) has seen a huge increase in the popularity of binary code similarity, with 50 approaches identified. The focus on this decade has been on binary code search approaches, with an emphasis since 2015 on its cross-architecture version (14 approaches), and in recent years on machine learning approaches.

In 2013, Wei et al. proposed RENDEZvous, a binary code search engine that given the binary code of a query function, finds other functions in a repository with similar syntax and structural properties. Reducing the search granularity from whole programs (SMIT) to smaller pieces of binary code such as functions enables an array of applications such as clone detection and bug search.

Most binary code search approaches target the bug search application. This application was first addressed on source code in 2012 by Jang et al. [138]. In 2014, David et al. proposed TRACY [62], the first binary code search approach focused on bug search. TRACY used the concept of tracelets, an execution path in a CFG, to find functions similar to a vulnerable function. In 2015, Pewny et al. presented MULTIMH [64], the first cross-architecture binary code search approach. MULTI-MH indexed functions by their input-output semantics. Given a function compiled for one CPU architecture (e.g., x86) MULTI-MH can find similar functions compiled for other architectures (e.g., MIPS). This problem quickly gained traction due to the popularity of embedded devices.

In 2016, Lageman et al. [109] trained a neural network to decide if two functions were compiled from the same source code. The use of deep learning has picked up in the last two years, e.g., $\alpha$ DIFF (2018), INNEREYE (2019), and ASM2VEC (2019). 


\subsection{Applications}

This section motivates the importance of binary code similarity by describing the applications it enables. Of the 59 papers analyzed, 36 demonstrate an application, i.e., present a quantitative evaluation, or case studies, of at least one application. The other 23 papers present generic binary code similarity capabilities that can be used for multiple applications such as binary diffing tools (e.g., [58, 59, 139]), binary code search platforms (e.g., [100, 108, 109]), and binary clone detection approaches (e.g., [95, 102, 110, 111]). Table 3.2 summarizes the eight applications identified. Most of the 36 papers demonstrate a single application, although a few (e.g., F2004 [26], BINSEQUENCE [69]) demonstrate multiple. One property of an application is whether the application compares different versions of the same program (patch analysis, patch generation, porting information, malware lineage), different programs (malware clustering, malware detection, software theft), or can be applied to both cases (bug search). Next, we detail those applications.

1. Bug search - Arguably the most popular application of binary code similarity is finding a known bug in a large repository of target pieces of binary code [62-75]. Due to code reuse, the same code may appear in multiple programs, or even in multiple parts of the same program. Thus, when a bug is found, it is important to identify similar code that may have reused the buggy code and contain the same, or a similar, bug. Bug search approaches take as input a query buggy piece of binary code and search for similar pieces of binary code in a repository. A variant of this problem is cross-platform bug search, where the target pieces of binary code in the repository may be compiled for different platforms (e.g., x86, ARM, MIPS) [64, 65, 67, 68, 70-73, 75].

2. Malware detection - Binary code similarity can be used to detect malware by comparing a given executable to a set of previously known malware samples. If similarity is high then the input sample is likely a variant of a previously known malware family. Many malware detection approaches are purely behavioral, comparing system or API call behaviors (e.g., [123, 124]). However, as described in Section ??, we focus on approaches that use binary code similarity [80-82].

3. Malware clustering - An evolution of malware detection is clustering similar, known malicious, executables into families. Each family cluster should contain executables from the same malicious program, which can be different versions of the malicious program, as well as polymorphic (e.g., packed) variants of a version. Similar to malware detection, we focus on approaches that compare binary code $[76,77]$ and exclude purely behavioral approaches based on system calls and network traffic (e.g., [126, 140,141]).

4. Malware lineage - Given a set of executables known to belong to the same program, lineage approaches build a graph where nodes are program versions and 
edges capture the evolution of the program across versions. Lineage approaches are most useful with malware because version information is typically not available $[15,31,83,142]$. Since input samples should belong to the same family, malware lineage often builds on the results of malware clustering.

5. Patch generation and analysis - The earliest binary code similarity application, and one of the most popular, is to diff two consecutive, or close, versions of the same program to identify what was patched in the newer version. This is most useful with proprietary programs where the vendor does not disclose patch details. The diffing produces small binary code patches that can be efficiently shipped to update the program [94]. It can also be used to automatically identify security patches that fix vulnerabilities [87], analyze those security patches [26, $56,69,85,86,88$ ], and generate an exploit for the old vulnerable version [143].

6. Porting information - Binary code similarity can also be used for porting information between two close versions of the same program. Since close versions typically share a large amount of code, the analysis done for one version may be largely reusable for a newer version. For example, early binary code similarity approaches ported profiling data $[84,144]$ and analysis results obtained during malware reverse engineering $[26,85]$.

7. Software theft - Binary code similarity can be used for identifying unauthorized reuse of code from a plantiff's program such as the source code being stolen, its binary code reused (e.g., [54]), a patented algorithm being reimplemented without license, or the license being violated (e.g., GPL code in a commmercial application). Early approaches for detecting such infringements used software birthmarks, i.e., signatures that capture inherent functionality of the plaintiff program $[145,146]$. However, as described in Section ??, we exclude signature-based approaches and focus on approaches using binary code similarity [61].

\subsection{Approaches}

In this section, we systematize the approaches of binary code similarity, describing the Approach Characteristics columns in Table 3.1. We recommend the reader to print Table 3.1 in a separate page to have it in hand while reading this section.

\subsubsection{Comparison Type}

\section{Columns: Input Comparison; Approach Comparison}

This section discusses the type of comparison between the approach inputs, as well as the finer granularity comparisons that may be used by the approach. 
Input comparison. All 59 works analyzed compare their inputs to identify similarity. That is, no approach identifies identical inputs (since a hash suffices for that) or input equivalence since it is an undecidable problem [?] that can only be solved efficiently for small pieces of binary code. Thus, we classify binary code similarity approaches based on their input comparison as: one-to-one (OO, 20 approaches), one-to-many (OM, 28 approaches), and many-to-many (MM, 11 approaches). The dominance of $O M$ approaches is due to the high interest in binary code search in the last decade.

It is always possible to build an $O M$ or $M M$ approach from an $O O$ approach. For example, a simple implementation of an $O M$ approach is to compare the given query piece of binary code with each of the $n$ targets using an $O O$ approach that returns the similarity between both inputs. Then, simply rank the $n$ targets by decreasing similarity and return the top $k$ entries or the entries above a similarity threshold. However, most $O M$ approaches avoid this simple implementation since it is inefficient. The two main solutions to improve performance are extracting a feature vector from each input and storing the target pieces of binary code in a repository with indices. Obtaining a feature vector for each input allows to perform the feature extraction only once per input. This offers significant benefits when the feature extraction is expensive, e.g., in the case of BLEX whose feature extraction requires executing a piece of binary code multiple times with different inputs. Once the feature vectors have been extracted, a similarity metric between two feature vectors is used. This similarity metric is typically cheap to compute as feature vectors are often numerical or Boolean. The other solution used by $O M$ approaches is adding indices on a subset of the features in the feature vector. The indices can be used to reduce the number of comparisons by applying the similarity metric only between the feature vector of the input piece of binary code and the feature vectors of selected targets more likely to be similar.

Approach comparison. Most approaches use a single type of comparison: similarity (45 approaches), equivalence (5), and identical (2). Note that even if only one type of comparison is used in the approach, it may differ from the input comparison. For example, EXEDIFF looks for identical instructions in the process of diffing two programs. There are 12 approaches that use multiple comparison types at different granularities. Of those, six use identical comparison at finer granularities to quickly identify the same pieces of binary code (BMAT, DR2005, SWPQS2006, BINCLONE) or to reduce expensive comparisons such as graph isomorphism (SMIT, SPAIN). The other six use equivalence comparisons at finer granularities to capture semantic similarity.

\subsubsection{Granularity}

\section{Columns: Input Granularity; Approach Granularities}

We separate the input granularity from the granularities of the pieces of binary code compared in the approach (i.e., approach granularities) since it is common to use finer granularities (e.g., functions) to compare coarser input granularity (e.g., whole programs). 
We have identified 8 comparison granularities: instruction (I), set of related instructions $\left(\mathrm{I}^{*}\right)$, basic block $(\mathrm{B})$, set of related basic blocks $\left(\mathrm{B}^{*}\right)$, function $(\mathrm{F})$, set of related functions $\left(\mathrm{F}^{*}\right)$, trace $(\mathrm{T})$, and whole program $(\mathrm{P})$. Instruction, basic block, function, and whole program are standard granularities that require no explanation. Related instructions ( $\left.I^{*}\right)$ are either consecutive (e.g., n-gram) or share a property (e.g., data dependent). They may belong to different basic blocks, and even to different functions. For example, TRACY groups instructions that collectively impact an output variable. Related basic blocks $\left(\mathrm{B}^{*}\right)$ share structural properties (e.g., graphlets in a CFG) or belong to the same execution path. Basic blocks in a set may belong to the same or multiple functions. Related functions $\left(\mathrm{F}^{*}\right)$ implement a program component such as a library, a class, or a module. Trace granularity compares the execution trace of two binary programs on the same input.

The most common input granularity is whole program (26 approaches) followed by function (22) and related basic blocks (4). Whole program is the preferred input granularity for $O O$ approaches (16/20) since most binary code diffing approaches try to establish a one-to-one mapping between all functions in the input programs, and also for $M M(7 / 11)$ approaches that tend to cluster input programs. On the other hand, function is the preferred granularity for binary code search approaches (20/28). Another four binary code search approaches use $\mathrm{B} *$ to identify code reuse that covers only a subset of a function or crosses function boundaries.

The most common approach granularity is function ( 25 approaches) followed by basic block (19). The majority of approaches (46/59) use different input and approach granularities, i.e., use finer approach granularities to compare coarser input granularity. Most approaches with the same input and approach granularity perform function searches (10/13). The 11 approaches that perform equivalence comparisons do so at fine granularities due to its low efficiency: six have B granularity, five $\mathrm{I}^{*}$, and one F. Some approaches accumulate features at a fine granularity that are never directly compared and thus do not show in the approach granularities column. For instance, GEMINI accumulates basic block features to generate a numerical vector at function granularity. Thus, only functions are compared.

\subsubsection{Syntactic Similarity}

\section{Column: Syntactic similarity}

Syntactic approaches capture similarity of the code representation, more especifically they compare sequences of instructions. Most commonly, the instructions in a sequence are consecutive in the virtual address space and belong to the same function. The instructions in the sequence may first be normalized, e.g., considering only the mnemonic, only the opcode, or normalizing the operands into classes. We detail instruction normalization in Section 3.6.10 and simply refer to instruction sequences in the rest of this subsection.

The instruction sequences may be of fixed or variable length. Fixed-size sequences are obtained by sliding a window over the instruction stream, e.g., over the linearly- 
ordered instructions in a function. This process is characterized by the window size, i.e., the number of instructions in the sequence, and the stride, i.e., the number of instructions to slide the start of the window to produce the next sequence. When the stride is smaller than the window size, consecutive sequences overlap. When the stride is one, the resulting sequence is called an n-gram. For example, given the sequence of instruction mnemonics $\{$ mov, push, add $\}$ two 2-grams will be extracted: $\{$ mov, push $\}$ and $\{$ push, $a d d\}$. There are 8 works that use n-grams: IDEA, BitSHrED, MBC, RENDeZvous, MutantX-S, Exposé, ILIne, and Kam1n0. Fixed-size sequences are also used by SWPQS2006 with a configurable stride larger than one. RENDEZVOUS, in addition to n-grams, also uses n-perms, unordered n-grams that capture instruction reordering within the sequence. An n-perm may capture multiple n-grams, e.g., 2-perm $\{$ mov, push $\}$ captures 2-grams $\{$ mov, push $\}$ and $\{$ push, mov $\}$.

The most common methods to compare instruction sequences are hashing, embedding, and alignment. Hashing is used by 6 approaches (BMAT, DR2005, SWPQS2006, SMIT, BINCLONE, SPAIN) to obtain a fixed-length value out of a variablelength instruction sequence. If the hash values are the same, the sequences are similar. Five approaches generate an embedding from n-gram sequences (IDEA, MBC, MutantX-S, Exposé, Kam1n0). Three approaches (Exediff, TraCy, BinSeQUENCE) align two sequences to produce a mapping between them by inserting gaps in either sequence to account for inserted, removed, and modified instructions. These approaches define a similarity score when instructions are aligned, and a gap score when an instruction aligns with a gap. Other less common comparison methods are using vectors of Boolean features (BITSHRED, ILINE) and encoding sequences as strings for indexing (RENDEZVOUS).

\subsubsection{Semantic Similarity}

Column: Semantic similarity
Semantic similarity captures if the code being compared has similar effects, as opposed to syntactic similarity that captures similarity in the code representation. The semantics of a piece of binary code can be described by the changes it produces in the process state, i.e., updates to the content of registers and memory. We identify 25 approaches computing semantic similarity. Most of them capture semantics at basic block granularity because basic blocks are straight-line code without control flow. Three methods are used to capture semantics: instruction classification, input-output pairs, and symbolic formulas.

Instruction classification. The first approach to introduce semantics into binary code similarity was KKMRV2005, which classified instructions into 14 classes (e.g., arithmetic, logic, data transfer) and used a 14-bit value to capture the classes of the instructions in a basic block. This semantic color bitvector captures the effects of the basic block. This approach was later adopted by BMM2006, BEAGLE, FossiL, and SIGMA. Instruction classification can be used to compute semantic similarity, but cannot determine if two pieces of binary code are, or are not, equivalent. 
Input-output pairs. Intuitively, two pieces of binary code are functionally equivalent if given the same input they produce the same output, for all possible inputs. Such equivalence is independent of the code representation and compares the final state after the code is executed, ignoring intermediate program states. This approach was proposed by Jiang et al. on source code [147] and later used by numerous binary code similarity approaches: BinHaSh, MXW2015, BleX, Multi-MH, BinGo, CACOMPARE, SPAIN, and KS2017. It involves executing both pieces of binary code with the same input and comparing their output, repeating the process many times. If the output differs for any input, then the two pieces of binary code are not equivalent. Unfortunately, to determine that both pieces are equivalent, the approach would require testing all possible inputs, which is not realistic for any non-trivial piece of binary code. Thus, in practice, this approach can only determine that two pieces of binary code are likely equivalent, with a confidence proportional to the fraction of inputs that have been tested, or that they are not equivalent (with certainty). The tested inputs are typically selected randomly, although it is possible to use other selection rules, e.g., taking values from the program data section (CACOMPARE). It is generally a dynamic approach, but some approaches (e.g., MULTI-MH) evaluate concrete inputs on statically-extracted symbolic formulas.

Symbolic formula. A symbolic formula is an assignment statement in which the left side is an output variable and the right side is a logical expression of input variables and literals that captures how to derive the output variable. For instance, the instruction add $\circ$ eax, $\circ$ ebx can be represented with the symbolic formula EBX2 = EAX + EBX1 where EBX2 and EBX1 are symbols representing the values of the EBX register before and after executing the instruction. Eleven approaches use symbolic formulas: BinHunt, iBinHunt, BinHash, Exposé, Tracy, RMKNHLLP2014, Tedem, CoP, Multi-MH, EsH, and XMATCH. Of those, eight approaches extract symbolic formulas at basic block granularity, XMATCH and EXPOSÉ extract formulas for the return values of a function, and BINSIM extracts symbolic formulas from an execution trace that capture how the arguments of a system call were derived. Three methods are used to compare symbolic formulas: using a theorem prover to check for equivalence, comparing the semantic hash of the formulas to check for equivalence, and computing the similarity of the graph representation of the formulas.

Theorem prover - BINHUNT introduced the idea of using theorem provers such as STP [148] or Z3 [149] to check if two symbolic formulas are equivalent, i.e., whether the output variable always contains the same value after the execution of both formulas, assuming that the input variables share the same values. The main limitation of this approach is that it is computationally expensive because it can only perform pairwise equivalence queries, the solving time quickly increases with formula sizes, and the solver may fail to return an answer for some queries. Note that a piece of binary code may have multiple outputs (registers and variables in memory), each represented by its own symbolic formula. These approaches need to try all pair-wise comparisons and check if there exists a permutation of variables such that all matched variables contain 
the same value.

Semantic hashes - An alternative to using a theorem prover is to check if two symbolic formulas have the same hash, after normalizing the formulas (e.g., using common register names) and simplifying them (e.g., applying constant propagation). The intuition is that if the two symbolic formulas have the same hash they should be equivalent. Three approaches use semantic hashes: BinHash, BINJUICE, and GiTZ. Semantic hashes are efficient, but are limited in that it is possible for two equivalent formulas to have different hashes even after normalization and simplification. For example, reordering of symbolic terms in one of the formulas (e.g., due to instruction reordering) results in a different hash.

Graph distance - XMATCH and TEDEM represent the symbolic formula of a basic block as a tree, and compute their similarity by applying graph/tree edit distance. Computing the graph/tree edit distance is more expensive than comparing semantic hashes, but the graph representation has the advantage over semantic hashes that it can handle term reordering.

\subsubsection{Structural Similarity}

\section{Column: Structural similarity}

Structural similarity computes similarity on graph representations of binary code. It sits between syntactic and semantic similarity since a graph may capture multiple syntactic representations of the same code and may be annotated with semantic information. Structural similarity can be computed on different graphs. The three most common are the intra-procedural control flow graph (CFG), the inter-procedural control flow graph (ICFG), and the callgraph (CG). All three are directed graphs. In the CFG and ICFG, nodes are basic blocks and an edge indicates a control flow transition (e.g., branch, jump). Basic blocks in a CFG belong to a single function; each function has its own CFG. Basic blocks in the ICFG belong to any program function; there is one ICFG per program. In the CG, nodes are functions and edges capture caller-callee relationships.

The intuition behind structural approaches is that CG, ICFG, and CFGs are fairly stable representations whose structure varies little for similar code. Approaches that operate on the CG or ICFG have a whole program input granularity, while those that operate on CFGs may have function granularity, or use function similarity as a step towards whole program similarity. Structural similarity approaches may use labeled graphs. For example, F2004 and DR2005 use node labels in the CG to capture structural information about a function's CFG (e.g., number of instructions and edges). Other approaches label basic blocks in the CFG/ICFG with a feature vector that captures the semantics of the basic clock (e.g., KKMRV2005, BINJUICE) or its embedding (GENIUS, GEMINI, see Section 3.6.6). Edge labels can be used to capture the type of control flow transfer (BMM2006) or to aggregate the semantic labels of source and destination nodes (FOSSIL). 
Structural similarity is used by 27 approaches. 14 approaches operate only on CFGs; five on both CFGs and the CG; four only on the ICFG; and two only on the CG. There are also three approaches that use non-standard graphs: SIGMA proposes a semantic integrated graph that combines information from the CFG, CG, and register flow graph, while QSM2015 and LIBV use the execution dependence graph [150]. The remainder of this subsection discusses different approaches used to compute graph similarity.

(Sub)Graph isomorphism - Most structural similarity approaches check for variations of graph isomorphism. An isomorphism of two graphs $G$ and $H$ is an edgepreserving bijection $f$ between their node sets such that if any two nodes $u, v$ are adjacent in $G$, then $f(u)$ and $f(v)$ are also adjacent in $H$. Graph isomorphism requires that the node set cardinality is the same in both graphs, which is too strict for binary code similarity. Thus, approaches instead check for subgraph isomorphism, which determines if $G$ contains a subgraph isomorphic to $H$. Subgraph isomorphism is a known NP-complete problem. Other approaches check for the maximum common subgraph isomorphism (MCS), which finds the largest subgraph isomorphic to two graphs and is also NP-Complete. Given the high complexity of both problems, approaches try to reduce the number of graph pairs that need to be compared, as well as the size of the compared graphs. For example, DR2005 avoids comparing CFGs with the same hash (match) and CFGs with very different number of nodes and edges (unlikely match). IBINHUNT reduces the number of nodes to consider by assigning taint labels to basic blocks. Only nodes with the same taint label are considered in the subgraph isomorphism. For candidate graph pairs that pass the filtering, approximate algorithms are used that can be grouped into greedy and backtracking.

Greedy - These approaches perform neighborhood exploration. An initial set of matching nodes is first identified. Then, the matching is recursively expanded by checking only the neighbors (i.e., parents or children) of already matched nodes. BMAT, F2004, DR2005, LKI2013, Tedem, Multi-MH, KLKI2016, KAM1N0, BinSEQUENCE, and BINARM use this approach. A limitation of greedy algorithms is that early errors propagate, significantly reducing the accuracy.

Backtracking - Backtracking algorithms fix a wrong matching by revisiting the solution, and if the new matching does not improve the overall matching it is reverted (BMM2006, BinHunt, IBINHunt, MXW2015, QSM2015, DISCOVRE). Backtracking is more expensive, but can improve accuracy by avoiding local optimal matching.

Optimization. An alternative used by four approaches (SMIT, BINSLAYER, CXZ2014, GENIUS) is to model graph similarity as an optimization problem. Given two CFGs and a cost function between two basic blocks, they find a bijective mapping between the two CFGs with minimum cost. Such bipartite matching ignores graph structure, i.e., does not use edge information. To address this, SMIT and BINSLAYER assign lower cost to connected basic blocks. To perform the matching, SMIT, BINSLAYER, and CXZ2014 use the $O\left(n^{3}\right)$ Hungarian algorithm, while GENIUS uses a genetic al- 


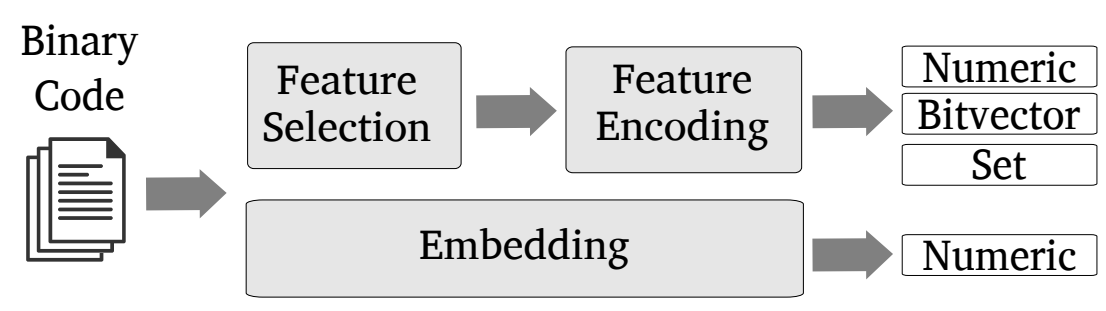

Figure 3.3: Two alternative methods for feature-based similarity.

gorithm.

K-subgraph matching. KKMRV2005 proposed to divide a graph into $k$-subgraphs, where each subgraph contains only $k$ connected nodes. Then, generate a fingerprint for each k-subgraph and the similarity of two graphs corresponds to the maximum number of $k$-subgraphs matched. Four other approaches later leveraged this approach: BEAGLE, CXZ2014, RENDEZVOUS, and FOSSIL.

Path similarity. There are three approaches (COP, SIGMA, BINSEQUENCE) that convert function similarity into a path similarity comparison. First, they extract a set of executions paths from a CFG, then define a path similarity metric between execution paths, and finally combine the path similarity into a function similarity.

Graph embedding. Another method used by GeniUs and GeMINI, detailed in Section 3.6.6, is to extract a real-valued feature vector from each graph and then compute the similarity of the feature vectors.

\subsubsection{Feature-Based Similarity}

\section{Column: Feature-based, Machine learning}

A common method (27 approaches) to compute similarity is to represent a piece of binary code as a vector or a set of features such that similar pieces of binary code have similar feature vectors or feature sets. A feature captures a syntactic, semantic, or structural property of the binary code. Features can be Boolean, numeric, or categorical. Categorical features have discrete values, e.g., the mnemonic of an instruction. A feature vector typically has all numeric or all Boolean features, the latter is called a bitvector. Categorical features are typically first encoded into Boolean features using one-hot encoding or into real-valued features using an embedding. Of the 27 approaches, 20 use numeric feature vectors, six use feature sets, and BINCLONE uses bitvectors. Once the features have been extracted, a similarity metric between feature vectors or feature sets is used to compute the similarity. Common similarity metrics are the Jaccard index for feature sets, dot product for bitvectors, and the Euclidean or cosine distance for numeric vectors.

Figure 3.3 shows two alternative methods for feature-based similarity. The top method (used by 21 approaches) comprises of two steps: feature selection and feature encoding. Feature selection is a manual process where an analyst uses domain 
knowledge to identify representative features. The alternative approach showed below is learning to automatically generate real-valued feature vectors, called embeddings, from training data. Embeddings are used by six recent approaches (GENIUS, GEMINI, $\alpha$ DIFF, RLZ2019, INNEREYE, ASM2VEC). Embeddings are used in natural language processing (NLP) to encode categorical features using real-valued numbers, which helps deep learning algorithms by reducing the dimensionality and increasing the density of feature vectors compared to one-hot encoding. Embeddings enable automatic feature extraction and efficient similarity computation. But, features in embeddings do not provide information about what has been learnt.

Binary code similarity embeddings can be classified by the properties they captured and their granularity. The first binary code similarity approach using embeddings was GENIUS, later followed by GEMINI. Both build a graph embedding for the ACFG of a function, i.e., a CFG with nodes annotated with selected basic block features. While GENIUS uses clustering and graph edit distance to compute the embedding, GEMINI improves efficiency by training a neural network that avoids expensive graph operations. Later approaches ( $\alpha$ DIFF, RLZ2019, INNEREYE, ASM2VEC) avoid manually selected features by focusing on instruction, or raw byte, co-ocurrencence. In NLP, it is common to extract a word embedding that captures word co-occurrence (e.g., word2vec) and then build a sentence embedding that builds upon it. RLZ2019 and INNEREYE use an analogous approach by considering instructions as words and basic blocks as sentences. Also related is ASM2VEC that obtains a function embedding by combining path embeddings capturing instruction co-occurrence along different execution paths in the function. Instead of using instruction co-ocurrence, $\alpha$ DIFF computes a function embedding directly from the sequence of raw bytes of a function using a convolutional network.

Machine learning. We identify three uses of machine learning in binary code similarity approaches: (1) to generate an embedding as explained above, (2) to cluster similar pieces of binary code using unsupervised learning (BITSHRED, BINHASH, MutAntX-S, ILINE, RMKNHLLP2014, KLKI2016, GENIUS), and (3) to classify if the pieces of binary code are being compiled from the same source code (BINDNN). BINDNN is the first use of neural networks for binary code similarity. Instead of generating an embedding, BINDNN directly uses a neural network classifier to determine if two functions are compiled from the same source code. Surprisingly, BINDNN is not cited by later binary code similarity approaches including those using neural networks to build embeddings.

\subsubsection{Hashing}

\section{Column: Locality sensitive hashing}

A hash is a function that maps data of arbitrary size to a fixed-size value. Hash values are compact to store, efficient to compute, and efficient to compare, which makes them great for indexing. Hashes operate at the raw-byte level. They are not especifically designed for binary code, but rather for arbitrary binary data. However, three 
classes of hashes have been used for binary code similarity: cryptographic hashes, locality-sensitive hashes, and executable file hashes. Cryptographic hashes capture identical, rather than similar, inputs. They are used by some binary code similarity approaches to quickly identify duplicates at fine granularity (e.g., basic block). Locality-sensitive hashes produce similar hash values for similar inputs, as oppossed to cryptographic hashes where a small difference in the input generates a completely different hash value. Executable file hashes take as input an executable file, but the hash computation only considers parts of the executable such as the import table or selected fields of the executable's header. Their goal is to output the same hash value for polymorphic variants of the same malware.

Locality sensitive hashing (LSH). LSH produces similar hash values for similar inputs, efficiently approximating a nearest neighbor search. LSH algorithms typically apply multiple hash functions on the given input and produce the same hash value for similar inputs, i.e., they increase collision probability for similar inputs. LSH is used in binary code similarity to boost performance. For example, it is used by $O M$ approaches for indexing pieces of binary code, enabling efficient binary code search (KAM1n0, Gemini, InNEREye). Of the 11 approaches that use LSH, seven use MinHash [?] (BinHash, Multi-MH, Genius, BinSequence, BinShape, CACOMPARE, BINSIGN), two do not specify the algorithm used (GEMINI, INNEREYE), SWPQS2006 uses the algorithm by Gionis et al. [?], and KAM1N0 proposes its own Adaptive Locality Sensitive Hashing (ALSH).

Fuzzy hashing is a popular type of LSH used to compute similarity of arbitrary files. For example, the VirusTotal file analysis service [151] reports ssdeep [128] hashes for submitted files. Other fuzzy hashes include tlsh [129], sdhash [152], and $m r s h-v 2$ [153]. None of the 59 approaches use them, but we briefly discuss them because they are often applied to executable files. When applied on executables, fuzzy hashes may capture similarity of the binary code, but also similarity of data present in the executables. This issue has been recently examined by Pagani et al. [154]. Their results show that when applied only on the code of the .text section they work significantly worse than when used for whole program similarity. In fact, they show that a byte change at the right position, extra nop instructions, and instruction swapping can degrade similarity significantly (in some cases bring it down to zero). They observe that when compiling the same source code with different optimizations, the data sections of the executables remain the same, which seems to be a key reason fuzzy hashes work better on whole executables.

Executable file hashes. This class of hashes are especifically designed for executable files. They are designed to output the same hash value for polymorphic variants of the same malware. The hash computation only considers parts of the executable that are less likely to change when simply repacking, or resigning, the same executable. They are not used by any of the 59 approaches, but we briefly describe three popular hashes for completeness. peHash [127] hashes selected fields of a PE executable that are less susceptible to changes during compilation and packing, e.g., initial stack size, 
heap size. ImpHash hashes the import table of an executable. Since the functionality of packed variants is the same, their imported functions should be the same as well. Unfortunately, it gives false positives with unrelated executables packed with the same packer, if the packer reconstructs the original import table at runtime. Authentihash is the hash of a PE executable ignoring its Windows Authenticode code signing data. It enables identifying the same executable signed by different publishers. It hashes the whole PE executable except three pieces: the Authenticode data, pointers to the Authenticode data, and the file checksum.

\subsubsection{Supported Architectures}

\section{Column: Cross-architecture}

A cross-architecture approach can compare pieces of binary code for different CPU architectures, e.g., x86, ARM, and MIPS. This differs from architecture-independent approaches (e.g., F2004) that support different architectures, but cannot cross-compare among them, i.e., they can compare two x86 inputs and two MIPS inputs, but cannot compare an x86 input with a MIPS input. There are 14 cross-architecture approaches, all proposed since 2015. A common application is given a buggy piece of binary code, to search for similar pieces of binary code, compiled for other architectures, which may also contain the bug. For example, to search for programs in firmware images where a version of OpenSSL vulnerable to Heartbleed has been statically compiled.

The code syntax for different architectures may significantly differ as they may use separate instruction sets with different instruction mnemonics, sets of registers, and default calling conventions. Thus, cross-architecture approaches compute semantic similarity. Cross-architecture approaches employ one of two techniques. Seven approaches lift the binary code to an architecture-independent intermediate representation (IR): Multi-MH, MockingBird, BinGo, XMATCH, CACOMPARE, GitZ, FIRMUP. Then, identical analysis can be peformeed on the IR, regardless of the origin architecture. The advantage is that the analysis only depends on the IR and the IR design can be outsourced to a separate group. Section ?? details the specific architectures supported by each approach and the IRs they use. An alternative approach used by 8 approaches is to use feature-based similarity (discussed in Section 3.6.6). These approaches use a separate module for each architecture to obtain a feature vector that captures the semantics of the binary code (DISCOVRE, BINDNN, $\alpha$ DIFF, ASM2VEC, INNEREYE, RLZ2019, GENIUS, GEMINI).

\subsubsection{Type of Analysis}

Column: Static analysis; Dynamic analysis; Dataflow analysis

Binary code similarity approaches can use static analysis, dynamic analysis, or both. Static analysis examines the disassembled binary code, without executing it. Instead, dynamic analysis examines code executions by running the code on selected 
inputs. Dynamic analysis can be performed online, as the code executes in a controlled environment, or offline on traces of the execution. Fifty approaches use only static analysis, three use only dynamic analysis, and six combine both. The dominance of static analysis for binary code similarity is due to most applications requiring all the input code to be compared. This is easier with static analysis as it provides complete code coverage. Dynamic analysis examines one execution at a time and can only determine similarity of the code run in that execution. To increase the coverage of dynamic analysis, two approaches (IBINHUNT, BLEX) run the code on multiple inputs covering different execution paths and combine the results. However, it is infeasible to run any non-trivial piece of binary code on inputs that cover all possible execution paths.

One advantage of dynamic analysis is simplicity, as memory addresses, operand values, and control-flow targets are known at runtime, which sidesteps static analysis challenges such as memory aliasing and indirect jumps. Another advantage is that it can handle some obfuscations and does not require disassembly of the code, also difficult with obfuscated code [155]. Section ?? details which approaches have evaluated its robustness on obfuscated code. Overall, dynamic analysis has been used in binary code similarity for malware unpacking (SMIT, BEAGLE, MutANTX-S, CXZ2014, BINSIM), for operating on trace granularity (BINSIM, KS2017), and for collecting runtime values for semantic similarity (BLEX, BINSIM).

Dataflow analysis is a common type of analysis that examines how values propagate through the code. It comprises of data sources to be tracked (e.g., registers or memory locations holding specific variables), propagation rules defining how values are propagated by different instructions or IR statements, and sinks, i.e., program points where to check the values reaching them. Of the 17 approaches that use dataflow analysis, 16 use symbolic execution to extract a set of symbolic formulas to compute semantic similarity (Section 3.6.4). SPAIN uses taint analysis to summarize the patterns of vulnerabilities and their security patches. And, IBINHUNT uses both taint analysis and symbolic execution. It first uses taint analysis as a filter to find pieces of binary code that process the same user input, restricting the expensive subgraph isomorphism computation to those with the same taint label. And, it computes basic block similarity using symbolic formulas.

\subsubsection{Normalization}

Column: Normalization

Syntactic similarity approaches often normalize instructions, so that two instructions that are normalized to the same form are considered similar despite some syntactic differences, e.g., different registers being used. Overall, there are 33 approaches that use instruction normalization. They apply the following three types of instruction normalization:

- Operand removal - A normalization used by nine approaches is to abstract an instruction only by its mnemonic or opcode, ignoring all operands. For example, 
add \%eax, \%ebx and add [\%ecx], \%edx would be both represented by add and considered similar, despite both using different operands.

- Operand normalization - A normalization used by 16 approaches is to replace instruction operands with symbols that capture the operand type such as $R E G$ for register, $M E M$ for memory, and $I M M$ for immediate values. For example, add\%eax, \%ebx and add\%ecx, \%edx would be both represented as add REG, REG, matching the instructions despite different register allocations used by the compiler. Operand normalization abstracts less than operand removal. For example, add [\%ecx], \%edx would be represented as add MEM, REG and thus be considered different from the above. Some approaches also use different symbols for general purpose registers and segment registers, and for operands of different sizes (e.g., RegGen8 and RegGen32 in BinClOnE).

- Mnemonic normalization - A normalization used by 3 approches (BMAT, EXPOSÉ and ILINE) is to represent multiple mnemonics by the same symbol. For example, both BMAT and ILINE represent all conditional jumps (e.g., je, jne) with the same symbol to account for the compiler modifying jump conditions.

Another type of normalization is to ignore code that should not affect the semantics. For example, some instruction sets contain no-op instructions that do not change the process state. And, compilers often use no-op equivalent instructions for padding such as instructions that move a register to itself, e.g., mov \%eax, \%eax. No-op equivalent instructions do not matter for semantic similarity and structural similarity approaches, but since they change the syntax they may affect syntactic similarity approaches. Three approaches remove no-op instructions (ILINE, MXW2015, QSM2015). A few approaches also remove unreachable dead code (BMM2006, FIRMUP), which may be introduced by obfuscations; function epilogue and prologue (EXPOSÉ) instructions, which may make small unrelated functions to look similar; and functions added by the compiler to load the program, not present in the source code (BINGo, SigMA).

\subsection{Implementations}

This section systematizes the implementation of the 59 approaches. For each approach, Table 3.3 shows the static and dynamic platforms it builds on, the programming language used to implement the approach, whether the implementation supports distributing the analysis, the supported target program architectures and operating systems, and how the approach is released. A dash (-) indicates that we could not find the information (i.e., unknown), while a cross $(\boldsymbol{X})$ means unsupported/unused.

Building a binary code similarity approach from scratch requires significant effort. Thus, all approaches build on top of previously available binary analysis platforms or tools, which provide functionality such as disassembly and control flow graphs for static analysis, or instruction-level monitoring for dynamic analysis. However, the 
Table 3.3: Comparison of the implementations of binary code similarity approaches. Symbol - means information is unknown.

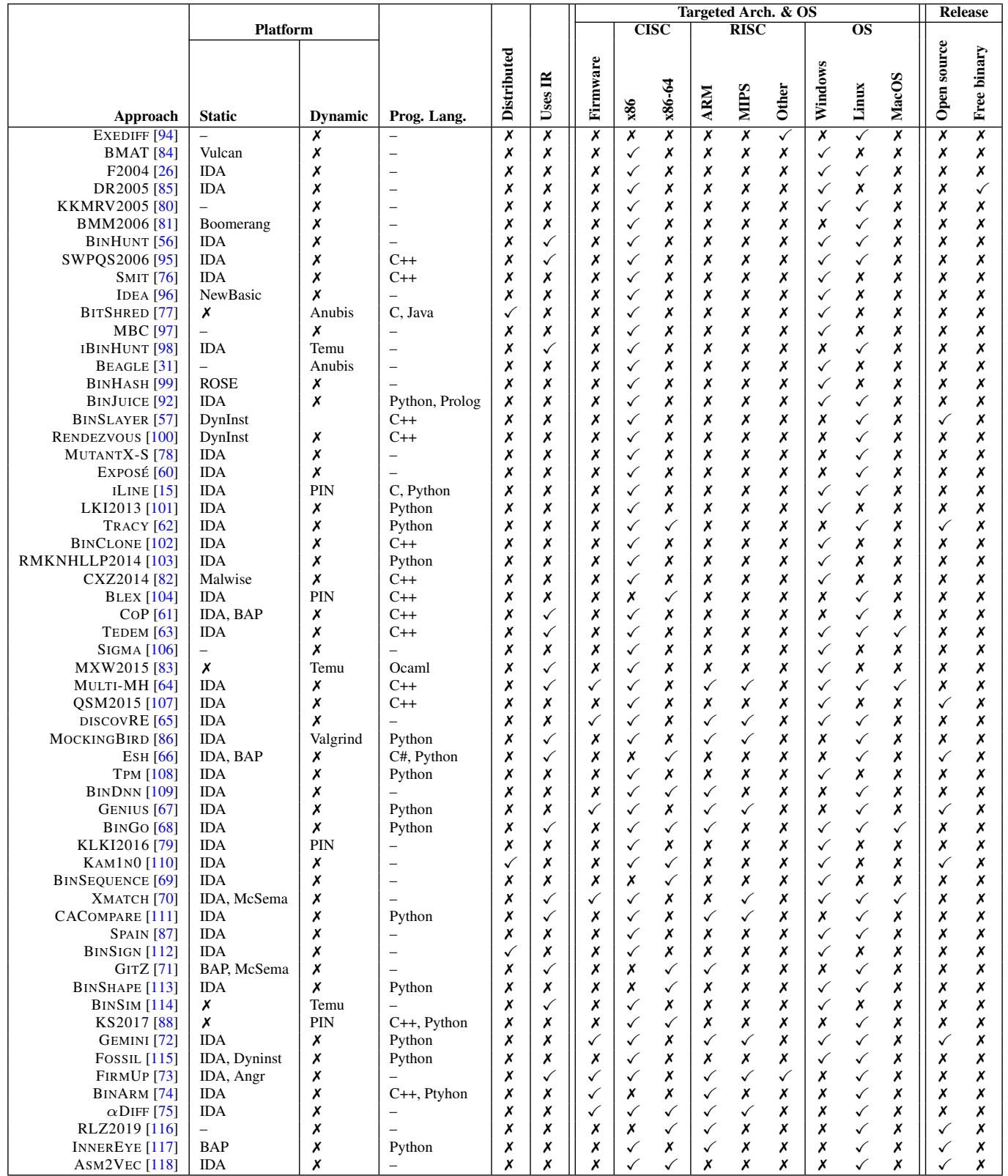


implementation of an approach may only use a subset of the functionality offered by the underlying platform. The most popular static platform is IDA (40 approaches), followed by BAP (5), McSema (3), and DynInst (2). IDA main functionalities are disassembly and building control flow graphs. Its popularity comes from supporting a large number of architectures. Some binary analysis platforms already support using IDA as their disassembler, so it is not uncommon to combine IDA and another platform (6 approaches). Among dynamic approaches, TEMU and ANUBIS are the most popular with 3 approaches each, followed by PIN (2). Previous work has analyzed binary analysis platforms used by binary code type inferece approaches [1]. Since most platforms overlap, we refer the reader to that work for platform details, but provide an extended version of their table in the Appendix (Table 5.1) with a couple of extra platforms.

A few approaches build on top of previous binary code similarity approaches. One case is that both approaches have overlapping authors. For example, RMKNHLLP2014 is based on BINJUICE, and MXW2015 extends IBINHUNT, which already shared components with BINHUNT. The other case is using a previously released approach. For example, BINSLAYER and SPAIN use BINDIFF as a first stage filter to find matched functions.

The most popular programming language is Python (17 approaches), followed by $\mathrm{C}++$ (13). One reason for this is that the majority of approaches use IDA for disassembly and IDA supports both $\mathrm{C}++$ and Python plugins. Moreover, 3 approaches (BITShred, KAM1N0, BinSign) have used distributed platforms, such as Hadoop, to distribute their analysis.

Binary code analysis can operate directly on a particular instruction set (e.g., x86, ARM) or convert the instruction set into an intermediate representation (IR). Using an IR has two main advantages. First, it is easier to reuse the analysis built on top of an IR. For example, supporting a new architecture only requires adding a new front-end to translate into the IR, but the analysis code can be reused. Second, complex instruction sets (e.g., x86/x86-64) can be converted into a smaller set of IR statements and expressions, which make explicit any side-effects such as implicit operands or conditional flags. There are 15 approaches that use an IR and most use the IR provided by the underlying platform. Out of 15 approaches, 5 use VINE provided by BITBLAZE (BINHunT, IBINHUNT, COP, MXW2015, BINSIM), another 5 use VEX provided with VAlgrind (Multi-MH, MOCKIngBird, CACOMPARE, GitZ, FirmUp), and two use LLVM-IR (ESH, XMATCH). The remaining three approaches use SAGE III (SWPQS2006), METASM (TEDEM) and REIL (BINGO). It is surprising that only 6 of the 14 cross-architecture approaches use an IR (MUlTI-MH, XMATCH, MOCKINGBIRD, CACOMPARE, GITZ, FIRMUP). The remaining approaches provide separate analysis modules for each architecture that typically output a feature vector with a common format.

The most supported architectures for the target programs to be analyzed are $\mathrm{x} 86 / \mathrm{x} 86$ 64 (55 approaches), followed by ARM (14), and MIPS (9). The only two approaches that do not support x86/x86-64 are EXEDIFF that targets Digital Alpha, and BINARM that targets ARM. There are 14 cross-architecture approaches and 7 approaches that 
support firmware. The first cross-architecture approach was MULTI-MH, which added support for ARM in 2015. Since then, ARM support has become very prevalent due to the popularity of mobile and IoT devices. It is worth noting that even if there are 40 approaches that use IDA, which supports more than 60 processor families, most approaches built on top of IDA only analyze x86/x86-64 programs. The most supported OS is Linux (38 approaches) followed by Windows (36). Only 4 approaches support MacOS. Early approaches that focused on x86/x86-64 often used IDA to obtain support for both PE/Windows and ELF/Linux executables. Most recently, all approaches that leverage ARM support Linux, which is used by Android and also by many IoT devices.

Of the 59 approaches, only 10 are open source (BINSLAYER, TRACY, QSM2015, Esh, Genius, Kam1n0, Gemini, Asm2Vec, InNerEye, RLZ2019). DR2005 was implemented in the BINDIFF commercial tool, which is now available as a free binary. The remaining approaches have not been released in any form, although the platforms they build on may be open source.

\subsection{Evaluations}

This section systematizes the evaluation of the 59 binary code similarity approaches. For each approach, Table 3.4 summarizes the datasets used (Section 3.8.1) and the evaluation methodology (Section 3.8.2).

\subsubsection{Datasets}

The left side of Table 3.4 describes the datasets used by each approach. It first shows the total number of executables used in the evaluation and their split into benign and malicious executables. Executables may come from different programs or correspond to multiple versions of the same program, e.g., with varying compiler and compilation options. Then, for approaches that have function granularity, it captures the total number of functions evaluated, and for approaches that analyze firmware, the number of images from where the executables are obtained. A dash (-) in a column means that we could not find the number in the paper. For example, SWPQS2006 evaluates on system library files in Windows XP, but the total number of executables is not indicated.

Most approaches use custom datasets, the one popular benchmark is Coreutils used by 15 approaches. In addition, approaches that evaluate on firmware use two openly available firmwares (ReadyNAS [156] and DD-WRT [157]). Over half of the approaches evaluate on less than 100 executables, 7 on less than $1 \mathrm{~K}, 8$ on less than $10 \mathrm{~K}$, and only 8 on over $10 \mathrm{~K}$. The largest dataset $(655 \mathrm{~K})$ is used by Bitshred, whose goal was scalability. Out of 59 approaches, 34 have evaluated only on benign programs, 9 only on malware, and 16 on both. This indicates that binary code similarity is also popular for malware analysis. However, of the 25 approaches evaluated on malware, only 
Table 3.4: Comparison of the evaluations of binary code similarity approaches. On the left it summarizes the datasets used and on the right the evaluation methodology. A dash (-) means we could not find the information. For Boolean columns, $\boldsymbol{X}$ indicates no support.

\begin{tabular}{|c|c|c|c|c|c|c|c|c|c|c|c|c|c|c|c|c|}
\hline \multirow[b]{2}{*}{ Approach } & \multicolumn{5}{|c|}{ Datasets } & \multicolumn{11}{|c|}{ Methodology } \\
\hline & 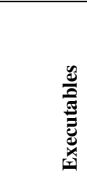 & 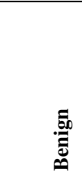 & 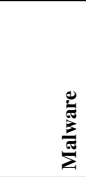 & : & 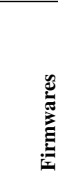 & نِ & $\underset{U}{U}$ & $\sum_{2}^{0}$ & 步 & 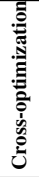 & 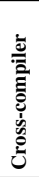 & 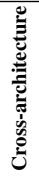 & 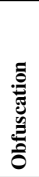 & 莺 & 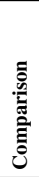 & 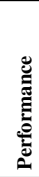 \\
\hline EXEDIFF [94] & 38 & 38 & 0 & 0 & 0 & $\checkmark$ & $x$ & $x$ & $x$ & $x$ & $x$ & $x$ & $x$ & $x$ & 1 & $x$ \\
\hline BMAT [84] & 32 & 32 & 0 & 0 & 0 & $x$ & $x$ & $\checkmark$ & $x$ & $x$ & $x$ & $x$ & $x$ & $\checkmark$ & 0 & $\checkmark$ \\
\hline F2004 [26] & 8 & 8 & 0 & 0 & 0 & $x$ & $x$ & $\checkmark$ & $x$ & $x$ & $x$ & $x$ & $x$ & $x$ & 1 & $\checkmark$ \\
\hline DR2005 [85] & 6 & 4 & 2 & 0 & 0 & $x$ & $x$ & $\checkmark$ & $x$ & $x$ & $x$ & $x$ & $x$ & $x$ & 0 & $\checkmark$ \\
\hline KKMRV2005 [80] & 503 & 61 & 442 & 0 & 0 & $\checkmark$ & $x$ & $\checkmark$ & $x$ & $x$ & $x$ & $x$ & $\checkmark$ & $\checkmark$ & 0 & $x$ \\
\hline BMM2006 [81] & 587 & 572 & 115 & 0 & 0 & $x$ & $x$ & $x$ & $x$ & $x$ & $x$ & $x$ & $x$ & $\checkmark$ & 0 & $\checkmark$ \\
\hline BinHunt [56] & 6 & 6 & 0 & 0 & 0 & $\checkmark$ & $x$ & $\checkmark$ & $x$ & $x$ & $x$ & $x$ & $x$ & $\checkmark$ & 0 & $\checkmark$ \\
\hline SWPQS2006 [95] & - & 1,723 & 0 & 0 & 0 & $\checkmark$ & $x$ & $\checkmark$ & $x$ & $\checkmark$ & $x$ & $x$ & $x$ & $\checkmark$ & 0 & $\checkmark$ \\
\hline $\begin{array}{l}\text { SMIT [76] } \\
\text { SM }\end{array}$ & 102,391 & 0 & 102,391 & 0 & 0 & $x$ & $x$ & $\checkmark$ & $x$ & $x$ & $x$ & $x$ & $\checkmark$ & $\checkmark$ & 0 & $\checkmark$ \\
\hline IDEA [96] & 26,189 & 13,000 & 13,189 & 0 & 0 & $x$ & $x$ & $\checkmark$ & $x$ & $x$ & $x$ & $x$ & $x$ & $x$ & 0 & $x$ \\
\hline BITSHRED [77] & 655,360 & 0 & 655,360 & 0 & 0 & $x$ & $x$ & $\checkmark$ & $x$ & $x$ & $x$ & $x$ & $x$ & $x$ & 2 & $\checkmark$ \\
\hline IBINHUNT [98] & 9 & 9 & 0 & 0 & 0 & $\checkmark$ & $x$ & $x$ & $x$ & $x$ & $x$ & $x$ & $x$ & $x$ & 1 & $\checkmark$ \\
\hline $\mathrm{MBC}$ [97] & 27 & 0 & 27 & 0 & 0 & $x$ & $x$ & $\checkmark$ & $x$ & $x$ & $x$ & $x$ & $x$ & $x$ & 0 & $x$ \\
\hline BEAGLE [31] & 381 & 0 & 381 & 0 & 0 & $x$ & $x$ & $\checkmark$ & $x$ & $x$ & $x$ & $x$ & $x$ & $\checkmark$ & 1 & $x$ \\
\hline BINHASH [99] & 16 & 0 & 16 & - & 0 & $x$ & $x$ & $\checkmark$ & $x$ & $x$ & $x$ & $x$ & $x$ & $x$ & 0 & $x$ \\
\hline BINJUICE [92] & 70 & 20 & 50 & 0 & 0 & $\checkmark$ & $x$ & $\checkmark$ & $x$ & $x$ & $x$ & $x$ & $x$ & $x$ & 0 & $x$ \\
\hline BINSLAYER [57] & 44 & 44 & 0 & 0 & 0 & - & - & - & - & $x$ & $x$ & $x$ & $x$ & $\checkmark$ & 1 & $\checkmark$ \\
\hline RENDEZVOUS [100] & 98 & 98 & 0 & $0.004 \mathrm{M}$ & 0 & $\checkmark$ & $x$ & $x$ & $\checkmark$ & $\checkmark$ & $\checkmark$ & $x$ & $x$ & $\checkmark$ & 0 & $\checkmark$ \\
\hline MutantX-S [78] & 137,055 & 0 & 137,055 & 0 & 0 & $x$ & $x$ & $\checkmark$ & $\checkmark$ & $x$ & $x$ & $x$ & $\checkmark$ & $\checkmark$ & 0 & $\checkmark$ \\
\hline EXPosÉ [60] & 3,075 & 3,075 & 0 & 0 & 0 & $\checkmark$ & $x$ & $x$ & $x$ & $x$ & $x$ & $x$ & $x$ & $\checkmark$ & 0 & $\checkmark$ \\
\hline ILINE [15] & 1,891 & 1,777 & 114 & 0 & 0 & $\checkmark$ & $x$ & $\checkmark$ & $x$ & $x$ & $x$ & $x$ & $x$ & $\checkmark$ & 0 & $\checkmark$ \\
\hline LKI2013 [101] & 20 & 16 & 4 & 0 & 0 & $x$ & $x$ & $\checkmark$ & $x$ & $x$ & $x$ & $x$ & $x$ & $\checkmark$ & 1 & $x$ \\
\hline TRACY [62] & - & - & 0 & $1.0 \mathrm{M}$ & 0 & $\checkmark$ & $x$ & $x$ & $x$ & $x$ & $x$ & $x$ & $x$ & $\checkmark$ & 0 & $\checkmark$ \\
\hline BinCLONE [102] & 90 & 18 & 72 & 0 & 0 & $x$ & $x$ & $\checkmark$ & $x$ & $x$ & $x$ & $x$ & $x$ & $\checkmark$ & 1 & $\checkmark$ \\
\hline RMKNHLLP2014 [103] & 936 & 0 & 936 & - & 0 & $x$ & $x$ & $\checkmark$ & $x$ & $x$ & $x$ & $x$ & $x$ & $\checkmark$ & 0 & $\checkmark$ \\
\hline CXZ2014 [82] & 16,999 & 1,601 & 25,398 & 0 & 0 & $x$ & $x$ & $\checkmark$ & $x$ & $x$ & $x$ & $x$ & $\checkmark$ & $\checkmark$ & 0 & $\checkmark$ \\
\hline BLEX [104] & 1,140 & 1,140 & 0 & $0.196 \mathrm{M}$ & 0 & $\checkmark$ & $\checkmark$ & $x$ & $\checkmark$ & $\checkmark$ & $\checkmark$ & $x$ & $x$ & $\checkmark$ & 1 & $\checkmark$ \\
\hline $\operatorname{CoP}[61]$ & 321 & 321 & 0 & 0 & 0 & $\checkmark$ & $\checkmark$ & $x$ & $x$ & $\checkmark$ & $\checkmark$ & $x$ & $\checkmark$ & $\checkmark$ & 4 & $x$ \\
\hline TEDEM [63] & 15 & 15 & 0 & - & 0 & - & $x$ & $\checkmark$ & - & $x$ & $x$ & $x$ & $x$ & $\checkmark$ & 0 & $\checkmark$ \\
\hline Sigma [106] & 18 & 16 & 2 & - & 0 & $x$ & $x$ & $\checkmark$ & $x$ & $x$ & $x$ & $x$ & $x$ & $x$ & 0 & $x$ \\
\hline MXW2015 [83] & 155 & 0 & 155 & 0 & 0 & $x$ & $x$ & $\checkmark$ & $x$ & $x$ & $x$ & $x$ & $x$ & $x$ & 0 & $\checkmark$ \\
\hline MuLTI-MH [64] & 60 & 60 & 0 & - & 2 & $\checkmark$ & $x$ & $x$ & $\checkmark$ & $\checkmark$ & $\checkmark$ & $\checkmark$ & $x$ & $\checkmark$ & 0 & $\checkmark$ \\
\hline QSM2015 [107] & 384 & 384 & 0 & $0.020 \mathrm{M}$ & 0 & $x$ & $x$ & $\checkmark$ & $x$ & $x$ & $x$ & $x$ & $x$ & $\checkmark$ & 1 & $\checkmark$ \\
\hline DISCOVRE [65] & 2,280 & 2,280 & 0 & $0.564 \mathrm{M}$ & 2 & $\checkmark$ & $\checkmark$ & $\checkmark$ & $\checkmark$ & $\checkmark$ & $\checkmark$ & $\checkmark$ & $x$ & $\checkmark$ & 2 & $\checkmark$ \\
\hline MockINGBIRD [86] & 10 & 10 & 0 & - & 0 & $\checkmark$ & $x$ & $x$ & $\checkmark$ & $\checkmark$ & $\checkmark$ & $\checkmark$ & $x$ & $\checkmark$ & 2 & $\checkmark$ \\
\hline ESH [66] & 1,000 & 1,000 & 0 & - & 0 & $\checkmark$ & $\checkmark$ & $x$ & $\checkmark$ & $x$ & $\checkmark$ & $x$ & $x$ & $\checkmark$ & 2 & $\checkmark$ \\
\hline ТРM [108] & 7 & 0 & 7 & 0 & 0 & $x$ & $x$ & $\checkmark$ & $x$ & $x$ & $x$ & $x$ & $\checkmark$ & $\checkmark$ & 4 & $x$ \\
\hline BINDNN [109] & - & 2,068 & 0 & $0.013 \mathrm{M}$ & 0 & $\checkmark$ & $\checkmark$ & $x$ & $x$ & $\checkmark$ & $\checkmark$ & $\checkmark$ & $x$ & $\checkmark$ & 1 & $\checkmark$ \\
\hline GENIUS [67] & - & 17,626 & 0 & $420 \mathrm{M}$ & 8,128 & $\checkmark$ & $x$ & $x$ & $\checkmark$ & $\checkmark$ & $\checkmark$ & $\checkmark$ & $x$ & $\checkmark$ & 3 & $\checkmark$ \\
\hline BinGo [68] & 110 & 110 & 0 & $0.127 \mathrm{M}$ & 0 & $\checkmark$ & $x$ & $x$ & $\checkmark$ & $\checkmark$ & $\checkmark$ & $\checkmark$ & $x$ & $\checkmark$ & 4 & $\checkmark$ \\
\hline KLKI2016 [79] & 350 & 30 & 320 & 0 & 0 & $x$ & $x$ & $\checkmark$ & $x$ & $x$ & $x$ & $x$ & $x$ & $x$ & 1 & $\checkmark$ \\
\hline KAM1 N0 [110] & 10 & 10 & 0 & - & 0 & - & - & - & - & $x$ & $x$ & $x$ & $x$ & $x$ & 4 & $\checkmark$ \\
\hline BINSEQUENCE [69] & 19 & 17 & 2 & $3.2 \mathrm{M}$ & 0 & $x$ & $x$ & $\checkmark$ & $x$ & $x$ & $x$ & $x$ & $x$ & $\checkmark$ & 4 & $\checkmark$ \\
\hline ХМАТСН [70] & 72 & 72 & 0 & $0.007 \mathrm{M}$ & 1 & $\checkmark$ & $x$ & $x$ & $\checkmark$ & $x$ & $\checkmark$ & $\checkmark$ & $x$ & $\checkmark$ & 4 & $\checkmark$ \\
\hline CACOMPARE [111] & 72 & 72 & 0 & - & 0 & $\checkmark$ & $x$ & $x$ & $\checkmark$ & $\checkmark$ & $\checkmark$ & $\checkmark$ & $x$ & $\checkmark$ & 3 & $\checkmark$ \\
\hline Spain [87] & 28 & 28 & 0 & - & 0 & - & - & - & - & $x$ & $x$ & $x$ & $x$ & $\checkmark$ & 0 & $\checkmark$ \\
\hline BINSIGN [112] & 9 & 7 & 2 & $0.023 \mathrm{M}$ & 0 & $x$ & $x$ & $\checkmark$ & $x$ & $\checkmark$ & $x$ & $x$ & $\checkmark$ & $\checkmark$ & 2 & $\checkmark$ \\
\hline GitZ [71] & - & - & 0 & $0.5 \mathrm{M}$ & 0 & $\checkmark$ & $\checkmark$ & $x$ & $\checkmark$ & $x$ & $\checkmark$ & $\checkmark$ & $x$ & $\checkmark$ & 0 & $\checkmark$ \\
\hline BINSHAPE [113] & 51 & 50 & 1 & $3 \mathrm{M}$ & 0 & $\checkmark$ & $x$ & $\checkmark$ & $x$ & $\checkmark$ & $\checkmark$ & $x$ & $\checkmark$ & $\checkmark$ & 0 & $\checkmark$ \\
\hline BINSIM [114] & 1,062 & 4 & 1,058 & 0 & 0 & $x$ & $x$ & $\checkmark$ & $x$ & $x$ & $x$ & $x$ & $\checkmark$ & $\checkmark$ & 6 & $\checkmark$ \\
\hline KS2017 [88] & 11 & 11 & 0 & 0 & 0 & $\checkmark$ & $x$ & $x$ & $\checkmark$ & $\checkmark$ & $\checkmark$ & $x$ & $\checkmark$ & $\checkmark$ & 0 & $\checkmark$ \\
\hline GEMINI [72] & 51,314 & 51,314 & 0 & $420 \mathrm{M}$ & 8,126 & $\checkmark$ & $x$ & $x$ & $x$ & $\checkmark$ & $x$ & $\checkmark$ & $x$ & $\checkmark$ & 2 & $\checkmark$ \\
\hline FossiL [115] & 6,925 & 1,920 & 5,005 & $1.5 \mathrm{M}$ & 0 & $\checkmark$ & $\checkmark$ & $\checkmark$ & $\checkmark$ & $x$ & $x$ & $x$ & $\checkmark$ & $\checkmark$ & 7 & $\checkmark$ \\
\hline FIRMUP [73] & 200,000 & 200,000 & 0 & $40 \mathrm{M}$ & 2,000 & - & - & - & - & $x$ & $x$ & $\checkmark$ & $x$ & $\checkmark$ & 2 & $\checkmark$ \\
\hline BINARM [74] & 0 & 0 & 0 & $3.2 \mathrm{M}$ & 5,756 & $\checkmark$ & $x$ & $x$ & $x$ & $\checkmark$ & $x$ & $x$ & $x$ & $\checkmark$ & 5 & $\checkmark$ \\
\hline$\alpha$ DIFF [75] & 67,427 & 67,427 & 0 & 0 & 2 & $\checkmark$ & $x$ & $x$ & $\checkmark$ & $\checkmark$ & $\checkmark$ & $\checkmark$ & $x$ & $\checkmark$ & 6 & $x$ \\
\hline RLZ2019 [116] & - & - & 0 & $0.202 \mathrm{M}$ & 0 & $x$ & $x$ & $x$ & $\checkmark$ & $\checkmark$ & $x$ & $\checkmark$ & $x$ & $\checkmark$ & 0 & $\checkmark$ \\
\hline INNEREYE [117] & - & - & 0 & $1.2 \mathrm{M}^{*}$ & 0 & $x$ & $x$ & $x$ & $\checkmark$ & $\checkmark$ & $x$ & $\checkmark$ & $x$ & $\checkmark$ & 0 & $\checkmark$ \\
\hline ASM2VEC [118] & 1,116 & 1,116 & 0 & $0.140 \mathrm{M}$ & 0 & $\checkmark$ & $\checkmark$ & $x$ & $\checkmark$ & $\checkmark$ & $\checkmark$ & $\checkmark$ & $\checkmark$ & $\checkmark$ & 12 & $\checkmark$ \\
\hline
\end{tabular}


five use packed malware samples (SMit, BeAgle, MutantX-S, CXZ2014, BinSIM). These approaches first unpack the malware using a custom unpacker (SMIT) or a generic (write-and-execute) unpacker (BEAGLE, MutANTX-S, CXZ2014, BINSIM), and then compute binary code similarity. The rest have access to the malware's source code or to unpacked samples.

For binary code search approaches that use function granularity, the number of functions in the repository better captures the dataset size. The largest dataset is by GENIUS, which evaluates on 420M functions extracted from 8,126 firmwares. Prior approaches had evaluated on at most $0.5 \mathrm{M}$ functions, which demonstrates the scalability gains from its embedding approach. Five other approaches have evaluated on over 1M functions: Fossil (1.5M), BinSEQUence (3.2M), BinArm (3.2M), FirmUP (40M), and GEMini (420M), which uses the same dataset as GeNiUs. In addition, INNEREYE has evaluated on a repository of 1.2M basic blocks.

\subsubsection{Methodology}

The right side of Table 3.4 describes four aspects of the evaluation methodology used by each approach: robustness, accuracy, performance, and comparison with prior approaches.

Robustness. The first 8 columns on the right side of Table 3.4 capture how authors evaluate the robustness of their approaches, i.e., their ability to capture similarity despite transformations applied to the input programs. First, it shows whether they use each of the four compilers we have observed being employed to compile the programs in the dataset: GCC, ICC, Visual Studio (MSVS), and Clang. Then, it captures whether the authors evaluate similarity between programs compiled with different compilation options (cross-optimization), between programs compiled with different compilers (cross-compiler), and between programs compiled for different architectures (cross-architecture). Finally, it captures whether the authors evaluate similarity when obfuscation transformations are applied to the input programs.

There are 31 approaches that evaluate robustness (at least one $\checkmark$ in the last four robustness columns) and 28 that do not. Many early works did not evaluate robustness. This evaluation has become increasingly popular as approaches mature. The most popular robustness evaluation is cross-optimization (20 approaches), followed by cross-compiler (17), cross-architecture (15), and obfuscation (12). There is only one approach (ASM2VEC) that has evaluated on all four, and 9 approaches that have evaluated cross-optimization, cross-compiler, and cross-architecture. Approaches that evaluate cross-compiler also typically evaluate cross-optimization, as it is a simpler case. Simlarly, approaches that evaluate cross-architecture typically also evaluate cross-compiler, as cross-architecture programs may be produced using different compilers. Note that it is possible for approaches to compile programs with multiple compilers, but not perform cross-compiler evaluation, i.e., not compare similarity between programs compiled with different compilers. 
There are 12 approaches that have evaluated on obfuscated programs. Of those, two use only source code transformations (COP, ASM2VEC), three use only binary code transformations (KKMRV2005, TPM, BINSHAPE), five use packed malware (SMIT, BEAGLE, MutANTX-S, CXZ2014, BinSiM), and two evaluate both source code and binary code transformations (BINSIM, FOSSIL).

Accuracy. There are 46 approaches that perform a quantitative evaluation of their accuracy using some ground truth $(\checkmark)$, and 13 that perform qualitative accuracy evaluation through case studies $(\boldsymbol{X})$. Quantitative evaluation most often uses standard accuracy metrics such as true positives, false positives, precision, and recall. However, two approaches propose new application-specific accuracy metrics (ILINE, KS2017). Binary code search approaches find the most similar entries in a repository to a given piece of binary code. They may return multiple entries ranked in descending similarity order. They count a true positive if one of the top- $k$ most similar entries is a true match. Unfortunately, the values of $k$ vary across approaches and significantly impact the accuracy. Furthermore, many approaches do not describe the similarity threshold used to determine that no similar entry exists in the repository. Both issues make it difficult to compare their accuracy.

Performance. It is common (47/59) to measure the runtime performance of an approach. Runtime is typically measured end-to-end, but a few approaches report it for each approach component (e.g., BINSIM). Four approaches report their asymptotic complexity (BMM2006, SWPQS2006, ILINE, MOCKINGBIRD). In addition, two approaches report memory overhead (SWPQS2006, BITSHRED).

Comparison. There are 31 approaches compared with prior approaches. The focus of the comparison is accuracy, but 6 also compare runtime. The top target for comparison is BINDIFF (12 approaches compare with it). followed by TRACY (5), DISCOVRE (4), and MulTi-MH (4). Since most approaches are not publicly available, comparison is often performed by re-implementing previous approaches, which may require significant effort. One advantage of reimplementation is that approaches can be compared on new datasets. The alternative to re-implementation is to evaluate the new approach on the same dataset as a prior approach did, and compare with the reported results. This method is only used by 6 approaches (GENIUS, BINGo, XMATCH, CACOMPARE, GEMINI, and BINARM) likely because most datasets are custom and not publicly available.

\subsection{Discussion}

This section discusses open challenges and possible future research directions.

Small functions. Many binary code similarity approaches ignore small functions, setting a threshold on the minimum number of instructions or basic blocks a function should have to be considered. Oftentimes only functions with a handful of instructions, 
e.g., less than 5, are ignored, but some approaches use large thresholds like 100 basic blocks in TRACY. Small functions are challenging because they are common, may comprise of a single basic block that prevents structural analysis, and may have identical syntax despite different semantics. For example, setter functions that update the value of a field in an object have nearly identical syntax and structure, simply setting a memory variable with the value of a parameter. But, they may have very different semantics, e.g., setting a security level or updating a performance counter, Furthermore, semantic similarity techniques like instruction classification, symbolic formulas, and input-output pairs may fail to capture their differences, e.g., if they do not distinguish different memory variables. Similarity of small functions, and more generally of small pieces of binary code, remains an open challenge. One potential avenue would be to further incorporate context. Some structural approaches already consider the callgraph to match functions (e.g., F2004), but this does not always suffice. We believe that it may be possible to further incorporate other context like locality (e.g., how close the functions are in the program structure) or data references (e.g., whether they use equivalent variables).

Source-to-binary similarity. Some applications like plagiarism detection may require source code to be compared with binary code. Early approaches for source-tobinary similarity used software birthmarks that capture inherent functionality of source code $[145,146]$. Recently, source-to-binary similarity has been applied for searching if a known bug in open source code exists in some target binary code [120]. The availability of source code for one side provides more semantics (e.g., variable and function names, types, comments) potentially improving accuracy compared to simply compiling the source code and performing binary code similarity. We believe other applications remain that require determining if a target piece of binary code has been compiled, or has evolved, from some given source code. For example, there may be programs for which source code is only available for an old version and there is a need to understand how newer binary versions have evolved from the original source code.

Data similarity. This survey has focused on binary code similarity, but programs comprise both code and data. There may be situations where the data used by the code is as important as the code, e.g., when the only change between versions of a program is in the data used such as changing parameter values for a machine learning classifier. Furthermore, data may be stored in complex data structures that may be key to the functionality. There exists a long history of type inference techniques on binary code [1], which we believe could be combined with binary code similarity to compare the data structures used by different pieces of binary code, or how two pieces of binary code use the same data structure.

Scalability. The largest binary code similarity evaluation so far is by GENIUS and GEMINI on 420M functions from 8,126 firmware images (500K functions per image). While that is a significant step from prior approaches, if we consider instead $100 \mathrm{~K}$ unique firmware, a conservative number since it is expected that there will be 
20 Billion IoT devices connected to the Internet by 2020 [158], we need a binary code similarity approach that can handle 50 Billion functions. Thus, further improvements on scalability will be needed to realize the vision of binary code search engines.

Obfuscation. Many challenges still remain for binary code similarity on obfuscated code. For example, a recent study has shown that state of the art unpacking techniques can miss $20 \%-60 \%$ of the original code, e.g., due to incomplete function detection [142]. And, no binary code similarity approach currently handles virtualizationbased packers such as Themida [159] and VMProtect [160], which generate a random bytecode instruction set, transform an input piece of binary code into that bytecode instruction set, and attach an interpreter (or virtual machine) from the generated bytecode to native code. Furthermore, obfuscation is best addressed with semantic similarity, which has a challenge with obfuscation transformations that do not respect the original semantics of the code, but still perform its main goals. 


\section{Lineage Inference of Packed Malware}

In this chapter, we propose a novel malware lineage approach that works on malware samples collected in the wild. Given a set of malware executables from the same family, for which no source code is available and which may be packed, our approach produces a lineage graph where nodes are versions of the family and edges describe the relationships between versions. To enable our malware lineage approach, we propose the first technique to identify the versions of a malware family and a scalable code indexing technique for determining shared functions between any pair of input samples. We have evaluated the accuracy of our approach on 13 open-source programs and have applied it to produce lineage graphs for 10 popular malware families. Our malware lineage graphs achieve on average a 26 times reduction from number of input samples to number of versions.

\subsection{Introduction}

Malware lineage studies the evolutionary relationships among malware, which has important security applications in the context of malware analysis. For example, lineage can be a fundamental step for triage, labeling, categorization, threat intelligence, provenance, and authorship attribution. The goal of malware lineage is to produce a lineage graph where nodes are versions of the family and edges describe the ancestordescendant relationships between versions.

Similar to benign programs, malware families evolve to adapt to changing requirements by adding new functionality, and to improve stability by fixing bugs. However, malware development typically comprises of an extra step not present in benign software development. Once a new version of a malware family is ready, the malware authors pack the resulting executable to hide its functionality and thus bypass detection by commercial malware detectors. The packing process takes as input an executable and produces another executable with the same functionality. The packing process is typically applied many times to the same input executable, creating polymorphic vari- 
ants (i.e., executables) of exactly the same version, which look different to malware detectors.

An important open problem in malware lineage is identifying the versions of a malware family among a set of input executables belonging to the family. The study of malware lineage goes back over 20 years and multiple approaches have been proposed [8,14-24]. However, a persistent limitation of these approaches is that they consider every input sample a separate malware version. Thus, their output lineage graphs have a node for every input sample. This is problematic because the packing process produces many polymorphic variants of a malware version. Thus, many input samples should be represented by the same node in the lineage graph. This problem does not manifest in prior malware lineage approaches because they are evaluated on synthetic malware, malware that is not packed, or packed malware for which unpackers are readily available (e.g., UPX [25]). But, the majority of malware is packed and malware often uses custom packers for which off-the-shelf unpackers are not available [9]. Thus, version identification needs to be addressed with malware collected in the wild.

Identifying versions among packed samples from a malware family is a novel and challenging problem. This problem differs from program similarity [26] because two versions of the same family may be highly similar, but still different versions. For example, one version may patch the previous one by adding a conditional to fix an error condition. While the two versions may be nearly identical, they need to be represented by different nodes in the lineage graph. We address this problem by considering malware samples with the same set of functions as polymorphic variants, which should be represented by the same node in the lineage graph. Any changes in functionality between two samples such as adding a function, removing a function, or updating an existing function, means that both samples are from different versions, with their own nodes in the lineage graph.

Another challenge with malware collected in the wild is that we do not know how it was developed. Thus, we need a lineage inference algorithm that works independently of the development model used by the malware authors, e.g., straight-line, multiple independent lines, branching and merging. Unfortunately, ILINE [15], the stateof-the-art lineage inference algorithm, uses separate algorithms for straight-line and branching and merging development. Thus, ILINE requires knowing in advance the development model of the software to select the lineage algorithm. This is problematic with malware since the development model is not known.

Now, we propose a novel malware lineage approach that works on malware samples collected in the wild. Given a pool of malware executables from the same family, for which no source code is available and which may be packed with an unknown packer, it produces a lineage graph. Our malware lineage approach improves the stateof-art in two ways. First, we propose the first technique to identify the different versions of a malware family present in an input set of potentially packed executables. In our lineage graph, a node identifies a version of the malware family and represents all input samples that are polymorphic variants of that version, which greatly reduces the 
number of nodes compared to prior approaches that create one node per input sample. Second, we propose a novel lineage inference algorithm that works independently of the development model used by the malware, and that improves the accuracy compared to ILINE, the state-of-the-art lineage inference approach.

Performing lineage inference on malware samples collected in the wild requires addressing other challenges such as malware clustering [77, 140, 141, 161], unpacking $[10,12,13,30,162]$, and disassembly $[44,45]$. To address these challenges we adapt existing state-of-the-art solutions. In other words, our goal is not to propose novel malware clustering, unpacking, and disassembly techniques. Rather, we want to understand how far we can get using state-of-the-art techniques and to identify areas where improvements are needed. To this end, we propose two novel metrics to quantify the accuracy of the malware unpacking and disassembly process.

Our approach works as follows: Since malware executables are collected in the wild without labels indicating their family, we first cluster input executables into malware families. Then, each malware sample is unpacked using a generic dynamic unpacker. The unpacker recovers the original code as raw byte sequences in memory snapshots. To enable malware lineage, we need to represent the code in a form that enables further analysis. For this, we disassemble the unpacked malware code by removing code overlaps, identifying function boundaries, and applying dynamic information to improve disassembly results. This process outputs the unpacked and disassembled code as an IDA database [163], which serves as input to the lineage inference module that produces the lineage graph.

We have implemented our malware lineage approach and evaluated its accuracy on open-source programs, before applying it to 10 malware families. Our unpacking and disassembly modules recover up to $81 \%$ of the original functions of programs packed with 18 packers. The missing functions are properly unpacked, but the function identification misses their start addresses preventing their disassembly. We have evaluated our lineage inference module on 631 versions of 13 open-source programs, covering over 59 years of software development. Our lineage graphs achieve an average $95 \%$ accuracy, which improves on ILINE's results, without requiring apriori knowledge of the malware development model. We also show that our version identification technique has better accuracy and efficiency compared to using BINDIFF.

We have also evaluated our approach on 7,793 packed malware samples from 10 malware families. The generated lineage graphs show that our approach can handle different malware development models, i.e., straight line, $k$ independent lines, branching and merging. The lineage graphs succinctly summarize the evolution of a malware family, achieving an average 26 times reduction from number of input samples to number of versions. For example, the 1,354 SYTRO samples are grouped into 6 versions in the SYTRO lineage graph.

Our contributions are:

- We propose a novel approach for malware lineage that works with malware collected in the wild. It takes as input a set of samples from the same malware family and outputs a lineage graph that describes ancestor-descendant relation- 
ships among the family versions.

- We present the first technique for identifying the versions present in a pool of samples from the same malware family. Our technique classifies the input samples into versions, identifying polymorphic variants of the same version.

- We propose a novel lineage inference algorithm that works independently of the development process used by the malware and that improves the accuracy compared to the current state-of-the-art solution.

- We propose two metrics to quantify the accuracy of the unpacking and disassembly process.

- We evaluate the accuracy of our lineage approach on 13 open-source programs, achieving 95\% accuracy on the lineage graphs. We apply our approach to 10 malware families achieving a 26 times reduction from input samples to versions.

\subsection{Overview \& Problem Definition}

Our problem is given a set of malware samples from the same malware family collected in the wild, to build a lineage graph that captures the evolution of the malware family across versions. Section 4.2.1 details the challenges introduced by working on malware collected in the wild. Section 4.2.2 details the problem. And, Section 4.2.3 describes the version identification subproblem.

\subsubsection{Challenges}

The main requirement for our malware lineage approach is to handle malware samples collected in the wild. This entails the following challenges:

- Unknown versions. We ignore how many versions a malware family has, i.e., we do not know apriori the nodes in the lineage graph. Instead, we need to identify which input samples correspond to different versions and which are polymorphic variants of the same version. This is a key difference from our approach with respect to prior malware lineage approaches $[15,17-24]$ that consider each input sample as a distinct version.

- Unknown development model. We ignore how a malware family is developed. The current state-of-the-art malware lineage approach is ILINE [15], which proposes different malware lineage algorithms for different development models, e.g., one for straight-line and another for branching and merging. But, with malware we do not know apriori which development model has been used. By contrast, we propose a single lineage inference algorithm that works regardless of the development model used. 
- Unpacking. Malware is distributed as executables, i.e., without source code, and those executables are typically packed. Polymorphic variants are created by repacking the same version multiple times. Our approach unpacks the input malware executables to recover the original code.

- Disassembly. Malware disassembly is a difficult problem [45]. Our disassembly module addresses code overlaps, function identification, and uses dynamic information to guide the disassembly. It outputs the unpacked program as an IDA database.

- Unordered samples. The order in which malware samples are collected may not mirror the order in which their versions were developed. Thus, our lineage inference approach does not rely on collection timestamps.

\subsubsection{The Lineage Graph}

Given a set of malware samples $S=\left\{s_{1}, \ldots, s_{n}\right\}$ belonging to the same malware family, our approach outputs a lineage graph, i.e., a directed acyclic graph (DAG) $G=(V, E, F, L) . \quad V$ is the set of nodes and each node $v_{i} \in V$ corresponds to a different version of the malware family. Note that throughout the chapter we use node and version interchangeably. $E \subseteq V \times V$ is the set of edges, where an edge $v_{i} \rightarrow v_{j}$ indicates that $v_{j}$ was derived from $v_{i}$. The use of a DAG enables a version to be derived from multiple parent versions, which may happen when two development branches merge.

The function $F: V \rightarrow \mathscr{F}$ labels each node with the set of (unpacked) functions in that version, i.e., $F\left(v_{i}\right)=\left\{f_{1}, f_{2}, \ldots, f_{k}\right\}$. That is, we abstract a node by the set of functions in that version (output by $F$ ). Two nodes cannot have the same set of functions, i.e., $\forall i \neq j F\left(v_{i}\right) \neq F\left(v_{j}\right)$. Two nodes connected by an edge share at least one function, i.e., $v_{i} \rightarrow v_{j} \Rightarrow\left|F\left(v_{i}\right) \cap F\left(v_{j}\right)\right|>0$. Note that the function sharing property is not transitive. Path $v_{i} \rightarrow v_{j} \rightarrow v_{k}$ indicates that $v_{i}$ shares some functions with $v_{j}$ and $v_{j}$ shares some functions with $v_{k}$, but $v_{i}$ may not share any function with $v_{k}$.

The function $L: V \rightarrow 2^{S}$ labels each node with the set of input samples that correspond to that version. Thus, $L$ partitions $S$ into family versions, i.e., $\forall i \neq$ $j\left|L\left(v_{i}\right) \cap L\left(v_{j}\right)\right|=0 \wedge \sum_{i}\left|L\left(v_{i}\right)\right|=n$. The number of input samples of a version $\left|L\left(v_{i}\right)\right|$ intuitively captures the type of the version. For example, nodes representing a large number of input samples are likely to be major versions, while nodes representing a few samples may indicate beta versions where some new functionality is being tested.

Figure 4.1 shows the lineage graph our approach builds from 131 input samples from the PICSYS malware family. Our lineage graph has 3 nodes, rather than 131 nodes (one per sample) if prior approaches were used. The node labels are $\left|F\left(v_{i}\right)\right|,\left|L\left(v_{i}\right)\right|$, i.e., the number of functions in the version and the number of input samples of that version. The root represents 5 input samples all with the same 16 functions. The 


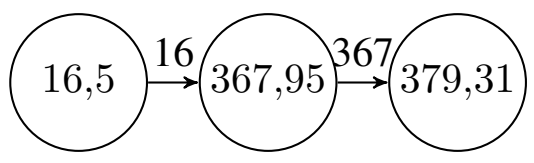

Figure 4.1: PICSYS lineage graph built using 131 samples. The node label represents the number of distinct functions in the version and number of samples of that version. The edge label is the number of shared functions.

version following the root represents 95 samples that have all 16 functions in the root and an additional 351 functions.

\subsubsection{Identifying Malware Versions}

Our intuition to address the version identification problem is that identifying versions in a pool of packed samples from the same malware family is analogous to finding clusters of semantically equivalent executables. On one hand, if two executables are variants of the same version they should be semantically equivalent, i.e., provide the same functionality, assuming that the compilation and packing toolchains are semanticspreserving. On the other hand, if two executables are semantically equivalent, then they implement exactly the same functionality and thus can be considered the same version. Note that we focus on differences visible in the binary code. If two versions simply refactor the source code by changing indentation or renaming variables, those changes are not visible in the executable. Thus, their executables have the same functionality and we would consider them the same binary version. That is, source versions are considered different binary versions only if they change the program functionality.

Unfortunately, current solutions to check if two executables are semantically equivalent are extremely expensive. In particular, BinHunt [56] requires between 30 minutes and 1 hour to semantically compare two consecutive program versions. Our smallest malware family has 113 samples, which would require over 6 months to be compared by BinHunt. Due to the quadratic number of comparisons, our largest family $(4,000$ samples) would take over 650 years. Other approaches perform semantic similarity $[104,164]$, but semantic similarity is not semantic equivalence because two versions of the same family may have highly similar functionality, but still are different versions, e.g., a version may simply add an error condition over the previous version.

To address scalability we leverage that if two functions have the same syntax, then their semantics are the same (but not vice versa). Thus, we perform a normalized syntactic matching between executables to efficiently identify re-packed variants of the same version. For this, we first tried using syntactic similarity tools [57,58]. But, those tools introduce errors for version identification because, similar to what happens with semantic similarity approaches, two versions of the same family may have highly similar syntax, but still are different versions. Thus, using these tools produces false positives, i.e., different versions identified as being the same. Furthermore, those tools perform pairwise comparisons, which create two problems for lineage inference. First, 


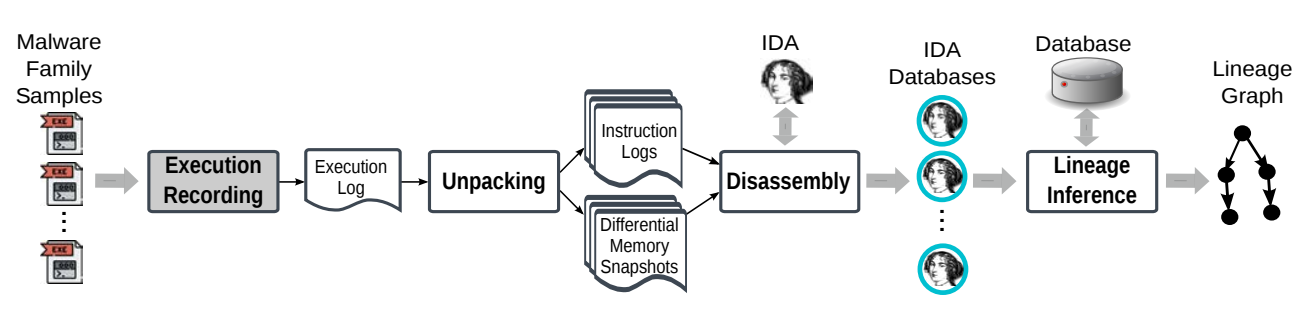

Figure 4.2: Approach overview. The execution recording and IDA tool are off-the-shelf components not developed in this work.

they do not scale well, e.g., after 8 hours BINDIFF only completes $14 \%$ of the pairwise comparisons for WINSCP's 47 versions. Second, to support branching and merging the lineage inference algorithm needs to identify functions that appear in a version, but do not appear in any predecessors (see Section 4.6). Such queries cannot be performed efficiently using pairwise comparisons, but require an indexing approach.

To address these limitations we use normalized function hashes, which allow us to efficiently identify the same function despite instruction or block reordering and the introduction of padding instructions that are semantically empty. The function hashes can be combined into a program hash that identifies samples that are the same version in $O(1)$. Function hashes also enable lineage inference to work because they allow us to identify in $O(1)$ all versions where a function is present.

Compilation toolchain. There are two cases in which we may create multiple nodes in the lineage graph for the same program version. First, if the same version is recompiled with different compilers or compilation options. Second, if the same version is packed with different packers. In essence, we consider the compilation and packing toolchains to define a version in addition to the source code. We believe both situations, while possible, are rare with real malware. For example, it makes little sense to use compilation options for evasion (when a packer is already used for this goal) since recompilation does not affect all functions and instructions, and thus may not bypass AV signatures. If malware authors recompile or repack the same version with different toolchains our approach may overestimate the number of versions. Still, compared to prior work, our approach achieves an order of magnitude reduction between number of input executables and number of versions in the lineage graph.

\subsection{Approach Overview}

Figure 4.2 summarizes our approach. It takes as input a set of program executables that belong to the same family and outputs a lineage graph. If the input malware samples are not labeled, as is often the case, we first cluster them using an off-theshelf malware clustering approach (not shown in the figure). In this work, we use the AVClass tool [165], which clusters and labels samples into known families using the AV labels in VirusTotal [151] reports. While AV labels are known to be noisy [93,166], 
AVClass successfully removes noise by performing label normalization, generic token detection, and alias detection. AVClass outputs for each sample the most likely family name and a confidence based on the agreement across AV engines.

Each family for which we want to analyze its lineage goes through processing depicted in Figure 4.2. For each sample in the family, our processing produces an output IDA database with the unpacked version of the sample's code. The first step in the processing of each sample is to run it using an execution recording module, which performs a lightweight recording of the execution that can be deterministically replayed as many times as desired. For this, we use the off-the-shelf QEMU-based PANDA record and replay platform [167]. PANDA's recording takes a snapshot of the VM state before execution and records non-deterministic changes to the CPU state and memory during execution. Such recording produces small changelogs and does not significantly slow down the VM (10\%-20\%), which limits time-related effects (e.g. network timeouts) on the target application. Using a record-and-replay platform enables separating the malware execution from the malware analysis. The small logs enable efficient storage of malware executions and the unpacking can be rerun every time an improvement is available.

The recording is used as input to the unpacking (Section 4.4) and disassembly (Section 4.5) modules. The unpacking module replays the recorded execution, while monitoring the instructions executed and the memory writes they perform. Every time a memory area is written and then executed it identifies a wave and takes a snapshot of the memory of the program. For each wave, the unpacking module outputs 2 wave files: a differential memory snapshot that stores the content of the memory regions overwritten and an instruction log with the unique instructions executed in the wave. Programs with only one wave are not packed.

The disassembly module takes as input the wave files produced by the unpacking and outputs an IDA database with the unpacked code. It comprises of 3 steps: code region identification, function identification, and instruction disassembly. For each wave, it first loads the memory ranges in the differential memory snapshot into the IDA database, relocating them to a different address if that range is already occupied by previously unpacked content. Then, it applies function identification to find the start address of functions present in the differential memory snapshot. Finally, it informs IDA to disassemble inside each loaded range starting at the instruction addresses in the instruction $\log$, and to create functions at the addresses identified by the function identification module.

The IDA databases for all samples in the family are input to the lineage inference module, which outputs the lineage graph (Section 4.6). The lineage inference module comprises of 3 phases. First, it computes the hashes of all functions in each IDA database and produces a program hash for each sample by hashing the concatenation of the function hashes. Second, it builds a lineage tree with the most likely parent for each node. Finally, it adds cross-edges to the lineage tree, by identifying if any node has additional parent nodes from which it inherits functions. This last step transforms the lineage tree into a lineage graph. 
We have implemented our malware lineage approach using over $11 \mathrm{~K}$ lines of $\mathrm{C} / \mathrm{C}++$ and scripts (Python and Bash), as measured by CLOC [168], i.e., without comments or blank lines. Of those, $5.8 \mathrm{~K}$ lines correspond to the PANDA unpacking plugin, $1.5 \mathrm{~K}$ to the IDA disassembly plugin, $3 \mathrm{~K}$ to the lineage module, and $1 \mathrm{~K}$ to scripts that glue the end-to-end processing.

\subsection{Unpacking}

We desire four properties from our unpacking module: (1) Support for both off-theshelf (e.g., Armadillo, PESpin) and custom packers. (2) Support for packers of different complexity (as defined by Ugarte et al. [9]). Specifically we want to support packers for which the unpacked original code may not be fully available in memory at any point of the execution, and samples that unpack code using multiple processes. However, we focus on packers that do not modify the original code, which excludes virtualization-based packers (e.g., Themida, VMProtect). (3) Maximize coverage, i.e., recover as much original code as possible. (4) Minimize noise, i.e., output as little code not part of the original code as possible.

We use the unpacking approach proposed by Bonfante et al. [10], which satisfies the first three properties mentioned above and to which we add differential memory snapshots to support the fourth property. Their approach runs the program and takes a memory snapshot before the first program instruction executes and at each wave change. Their wave semantics define when wave changes happen and guarantee that any code unpacked during execution will be present in a memory snapshot. Note that the unpacked code is a superset of the executed code, i.e., the memory snapshots typically contain much "dormant" original code, which was unpacked but not executed. For example, wave $W_{i}$ may unpack a function that contains a conditional. Only one branch of the conditional may be executed, but the code reachable through the other branch is also present in the memory snapshot taken before $W_{i+1}$ starts.

There are two main differences between [10] and our unpacking module. First, their unpacking code is not available and was built on top of Pin [169]. In contrast, our unpacking module is implemented as a plugin for the PANDA record and replay platform [167], which enables us to separate malware execution recording (which can be performed on dedicated malware farms $[170,171])$ from the unpacking. It also provides better malware isolation and allows the future support of other QEMU-supported architectures. Second, our unpacking minimizes the noise by producing a differential memory snapshot for each wave, in contrast with the process-level snapshot produced by Bonfante et al. The motivation for this is that a wave's snapshot shares much content with the snapshot of the prior wave. For example, the execution may first unpack a function $f_{1}$ and execute it (first wave), then unpack another function $f_{2}$ and execute it (second wave). In this case, the first snapshot contains $f_{1}$, while the second snapshot contains both $f_{1}$ and $f_{2}$. Removing the redundancy between snapshots reduces the noise in the IDA database output by the unpacking and disassembly modules. 
Unpacking overview. The unpacking module takes as input the execution log that records a run of a potentially packed program $P$. It replays the execution log and uses the wave semantics to identify waves in the list of tracked processes, which is initialized with the input process $P$ and to which any new process created (or injected from) a tracked process is added. At the beginning of the execution of each tracked process, a full memory snapshot of the process address space is taken. New waves are detected by monitoring, for each instruction executed by a tracked process, the memory ranges it may overwrite and whether previously overwritten bytes are being executed. For each new wave of a tracked process, the unpacking outputs a differential memory snapshot and a wave instruction log. The differential memory snapshot of wave $W_{i}$ stores the memory contents modified by the previous $W_{i-1}$ wave. It includes modified bytes in the main module, other modules, the heap, and the stack. The wave instruction log contains the instructions executed during the wave and marks instructions following calls as function entry points.

\subsection{Disassembly}

The unpacking module guarantees that the code the program unpacked during execution is present in the differential memory snapshots. However, it is present as part of a long sequence of raw bytes, which is not particularly useful. The goal of the disassembly module is given as input the instruction logs and differential memory snapshots produced by the unpacking, to output an IDA database where the instructions and functions in the unpacked code have been identified. IDA is arguably the most widely used reverse-engineering tool and already used by most malware analysts. Furthermore, popular IDA plugins exist for diffing two unpacked executables such as BinDiff [58] and Diaphora [59].

Next, we detail the three disassembly steps: memory range loading, function identification, and instruction disassembly.

Memory range loading. The disassembly combines all the unpacked code into the same IDA database. This includes code unpacked by all the waves observed during execution, from the original process executed as well as from any child process it may create. Intuitively, if all the code comes from the same input packed executable, then it should be available together to the analyst as if the input executable was not packed. Loading the contents of the differential memory snapshots into the IDA database comprises of three substeps: removing duplicate ranges, (optionally) selecting ranges, and relocating overlapping ranges. The use of differential memory snapshots prevents a memory range unpacked in wave $W_{i}$ to appear in the snapshots of waves $W_{i+1}, \ldots, W_{n}$. But, some packers (e.g., YodaProtector) unpack the same content on the same memory range again and again. This behavior would introduce many duplicates of the same content in the IDA database. To prevent this, when a memory range is loaded into the IDA database, its original address and the hash of its contents are logged. If a range has the same original address and contents of another range 
already loaded, it is a duplicate and can be skipped.

By default, all (non-duplicate) memory ranges in the differential memory snapshots are loaded into the IDA database. This includes ranges in the regions of the main module, other modules, heap, and stack. Some of those ranges may only contain data. An analyst, depending on the end application, may prefer to exclude data ranges to reduce the size of the final IDA database. The disassembly module allows an analyst to specify filters on which ranges should be loaded. For example, an analyst could specify that ranges on the stack or the heap should not be loaded, or that only ranges in pages with execution permission should be loaded.

While loading the selected memory ranges, the disassembly module monitors if two memory ranges overlap. If so, one of them needs to be relocated because IDA does not support having multiple contents at the same memory range. Internally, IDA uses a linear address space where analysts can create segments, which represent contiguous chunks in the linear address space. Creating a segment requires the start and end addresses of the segment in the linear address space, and the segment base. The segment base is used to compute virtual addresses for the segments, which are the addresses an analyst sees. The conversion from linear to virtual addresses is: VirtualAddress = LinearAddress - (SegmentBase $<<4)$. The disassembly module loads each range in each wave of each tracked process as a separate segment. It starts by creating a segment at the base address of the main module of the initial malware process, which corresponds to the contents of the packed malware sample. For each wave of the initial malware process, it creates another segment following the previous one in the linear address space ${ }^{1}$, adjusting the segment base so that virtual addresses in the new segment follow virtual addresses in the prior segment. When a wave is relocated, the disassembly module scans the disassembled instructions and rewrites any addresses found in the disassembled code to point to the same location as before the relocation. Once all waves of the initial process have been loaded, the memory range loading repeats for any other tracked processes.

Function identification. An important step when disassembling a program is identifying functions. Our disassembly module uses two techniques to locate function entry points and instructs IDA to create functions at those addresses. First, it uses the instructions marked in the wave instruction log to have followed a call instruction. This helps identify targets of indirect calls that appeared in the execution. Second, it uses ByteWeight [52] to locate the start address of functions in each of the regions loaded into the IDA database. This method can identify the entry point of functions that did not execute during unpacking. However, ByteWeight identifies functions in the text section of an input executable. Instead, we want to identify functions in the raw regions of memory contained in the differential memory snapshots. Thus, we have modified ByteWeight to take as input a sequence of assembly instructions, rather than a full executable. Our disassembly module first disassembles 10 instructions starting at each offset in a region and inputs those instructions to the modified ByteWeight, which

\footnotetext{
${ }^{1}$ Segments are separated by a 16 byte gap
} 
outputs a score between 0 and 1 indicating how likely the disassembled instructions correspond to a function start. As recommended in [52], the address of any sequence of instructions with a score higher than 0.5 is considered a function start.

Instruction disassembly. The instruction disassembly leverages the function start addresses identified by ByteWeight and dynamic information from the execution, e.g., to identify targets of executed indirect jumps. First, our disassembly module instructs IDA to start disassembling at every identified function start address. Then, it instructs IDA to start disassembling at every instruction in the wave instruction logs. For this, the information in the instruction $\log$ is used to identify which wave unpacked the instruction, which is used to locate the IDA segment where the instruction has been loaded. Note that when IDA is instructed to disassemble at an address, it performs a recursive disassembly to find as much code as possible. Thus, it also disassembles dormant code captured in the differential memory snapshots, which did not execute during the execution recording.

\subsection{Lineage Inference}

The lineage inference module takes as input the IDA databases of the family samples with the unpacked and disassembled code and produces the lineage graph. It comprises of three phases. The first phase identifies the nodes in the lineage graph (Section 4.6.1) and also constructs the $L$ and $F$ functions (see Section 4.2). The other two phases identify the edges in the lineage graph. The second phase builds a lineage tree starting with a selected root and greedily inserting the most similar node, not yet in the tree, to any node already in the tree (Section 4.6.2). In the lineage tree each node has at most one parent. The third phase identifies whether some nodes inherit code from multiple nodes and thus need to have more than one parent (Section 4.6.3). The complexity analysis of the three phases is provided in Section 4.6.4.

\subsubsection{Phase I: Identifying Versions}

Identifying the set of nodes in the lineage graph requires finding which input samples correspond to each family version. In our approach, two input samples are the same version if they have the same set of functions. To uniquely identify a function we use a function hash. The use of a function hash is critical for scalability. It enables identifying all samples that contain a specific function in $O(1)$, a fundamental step in phase III.

Our approach works with different function hashes. Each hash can have different properties and may produce a different set of nodes for the lineage graph. Overall, we seek function hashes that determine that two functions are the same with low false positives, and are efficient to compute and index. Currently, our approach supports two such function hashes: 
Raw hash. Performs an MD5 hash of the sequence of raw byte values from function start to function end. Two functions with the same raw hash have the same bytes and thus are the same function. This hash does not require disassembly and should not produce false positives. On the other hand, it can have high false negatives when modifications have been applied to the function that do not affect its functionality, e.g., semantics-preserving instruction reordering. We use this hash as baseline for comparison.

SPP-NOP hash. The small prime product (SPP) assigns to each instruction mnemonic a small prime number. The hash corresponds to the product of the primes of all mnemonics of the instructions in the disassembled function [85]. We have modified the SPP hash computation to ignore instructions used for padding that do not affect the function semantics, e.g., the no-op (NOP) instruction and a move from a register to the same register. The advantage of SPP-NOP over the raw hash is that it can detect the same function despite instruction reordering, block reordering, and instruction padding.

This phase first iterates on all the functions in each input IDA database, computing both the raw and SPP-NOP function hashes, and storing them in a central database. Short functions with at most two instructions are ignored. Then, it computes a raw program hash and a SPP-NOP program hash for each sample by sorting the (raw or SPP-NOP) function hashes for the sample, concatenating them separated by a delimiter, and hashing the concatenation using MD5. Each program hash represents one version in the lineage graph. Samples with the same program hash are polymorphic variants of the same version.

The raw and SPP-NOP hashes may produce different sets of nodes for the lineage graph. The raw hash gives an upper bound on the number of nodes since it should not have false positives. Thus, the number of nodes using the SPP-NOP hash is always less than or equal to the number of nodes using the raw hash. While they can produce different node sets, both hashes often agree. Specifically, in 10 out of 13 open-source programs and in 1 out of 10 malware families evaluated, both hashes produce the same set of nodes.

\subsubsection{Phase II: Building a Lineage Tree}

Given the nodes identified in Phase I, Phase II greedily builds a lineage tree where each node has a single parent. Its processing is described in Algorithm 1. Starting with an empty tree, it first inserts as root the node that minimizes the sum of its size (i.e., number of functions) and the average distance to all other nodes. This root selection is inspired by Lehman's $6^{\text {th }}$ software evolution law ("Continuing growth"), which states that programs tend to grow over time [172]. Then, it iterates inserting at each step the node, not yet in the tree, with the highest number of shared functions to any node already in the tree. The iteration terminates when all nodes have been inserted. 


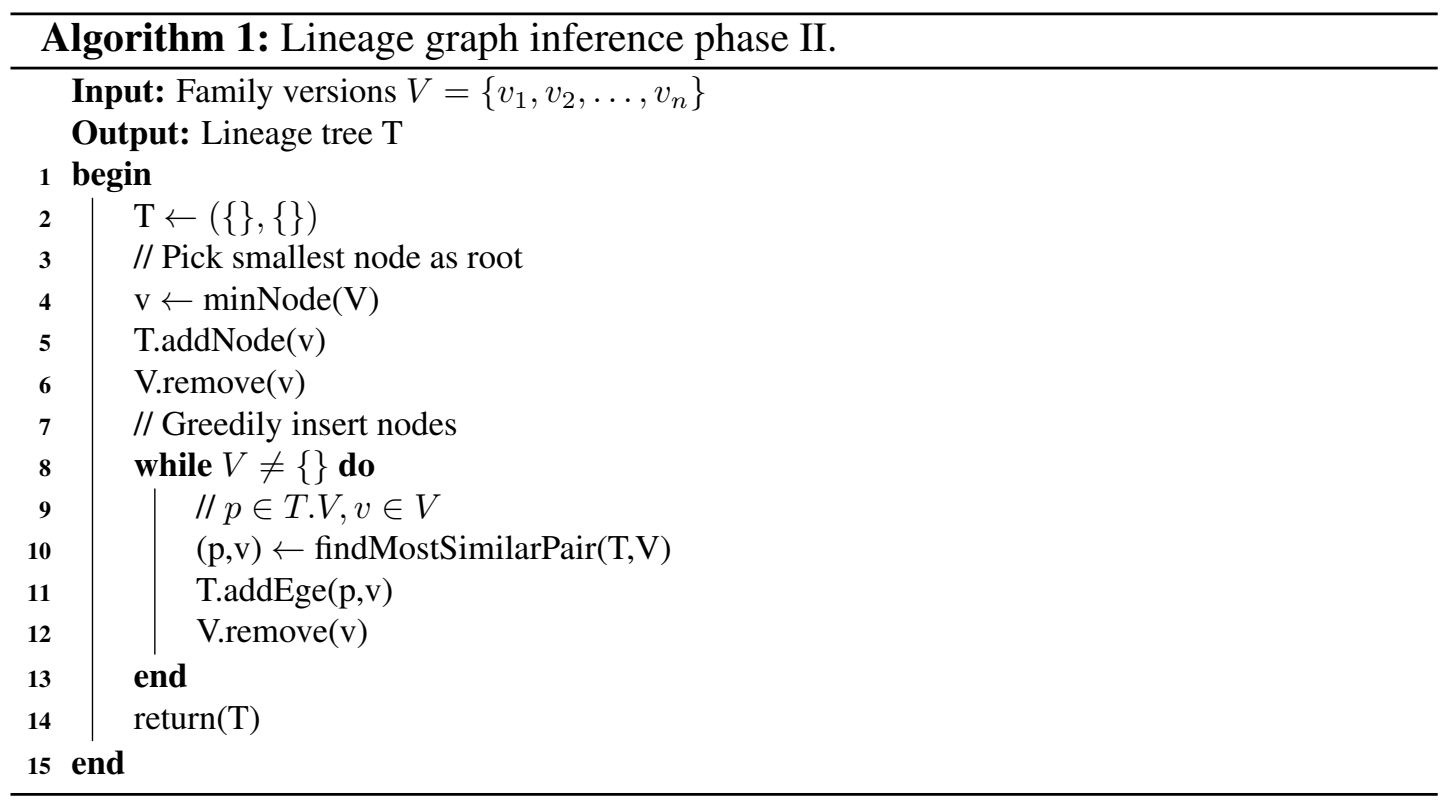

The function findMostSimilarPair greedily selects the next edge to insert and needs to handle two classes of ties. First, there could be multiple candidate nodes not yet in the tree that share the same number of functions with a node already in the tree. Here, the candidate with highest number of instructions shared with a node in the tree is selected. Second, the node to be inserted could share the same number of functions (or instructions) with multiple nodes already in the tree. Among those, the node latest inserted in the tree is picked as parent.

A special case happens when the remaining nodes, not yet in the tree, share very few (or no) functions with nodes already in the tree (we use an experimentally determined threshold of less than $2 \%$ similarity). This may indicate independent development lines or a large refactoring of the code. In this case, the algorithm picks the smallest node not yet in the tree as the next to insert, rather than the one most similar to a node in the tree (since none remaining are similar to those in the tree). An edge is added between the selected node and the most similar node in the tree. If the number of shared functions is zero, phase III will later remove those edges, thus introducing multiple independent lines.

\subsubsection{Phase III: Adding Cross-Edges}

Each node in the lineage tree has at most one parent. However, it is possible for a version to descend from multiple versions, e.g., when a development branch is merged back into the trunk. Phase III identifies versions with multiple parents.

Algorithm 2 describes this phase. The first step is to remove any edges with zero shared functions. This removal introduces additional roots, identifying independent development lines. Then, it iterates on the nodes in topological order. For the current 


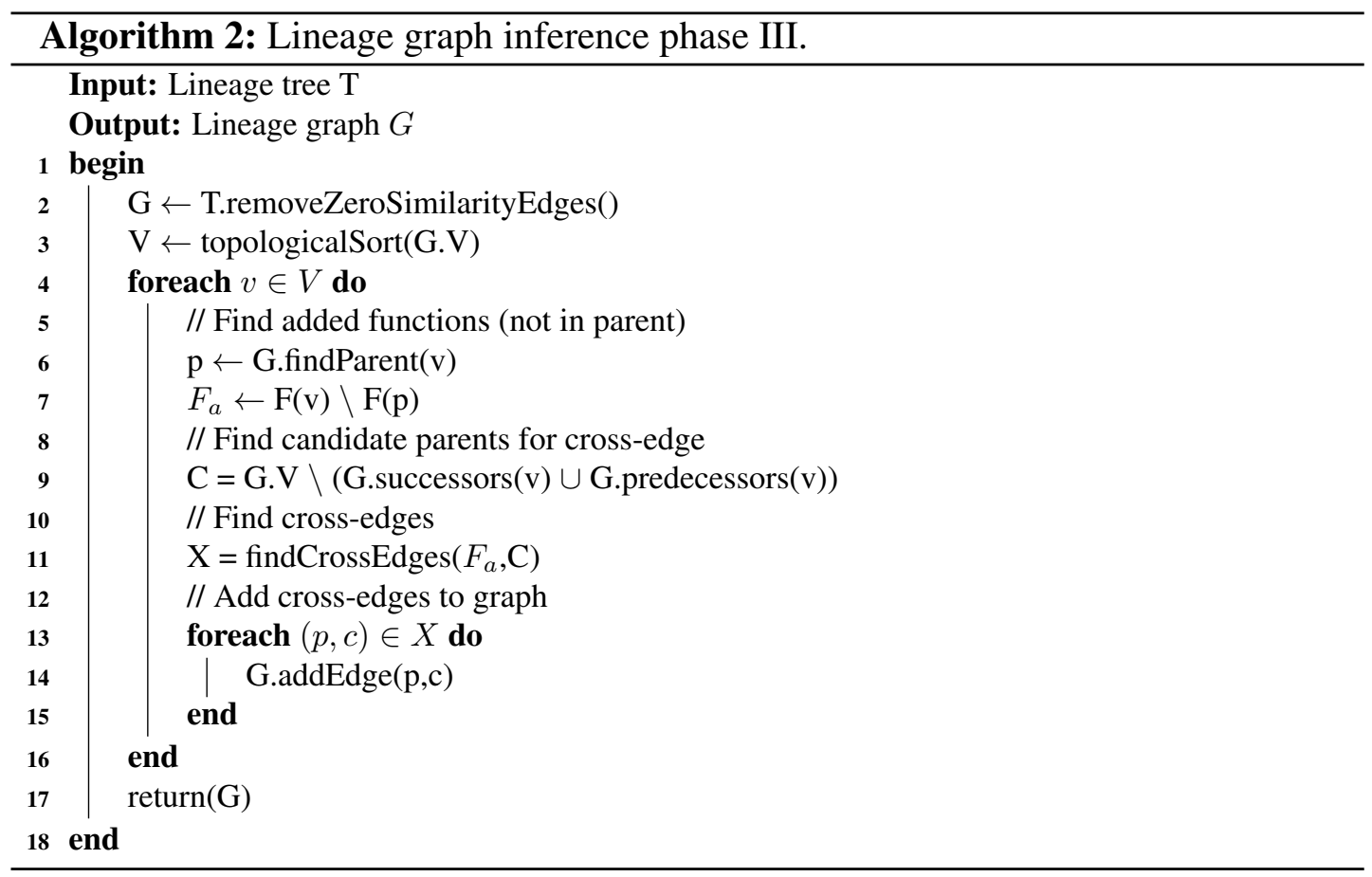

node, it first computes the set of added functions, i.e., functions in this version that are not inherited from the parent version. Then, it selects the candidate parents for a crossedge, which are the nodes that are not successors or predecessors of the current node. Successors are ignored because a cross-edge from them would introduce a cycle in the graph. Predecessors are ignored because they already influence the current node. Function findCrossEdges picks among the parent candidates the one that shares the most added functions with the current node. Then it removes those functions from the set of added functions and picks the next node that shares the most remaining added functions. The process terminates when no candidates share more than a threshold $t$ of functions, which we have determined experimentally to be 3. Once findCrossEdges returns, the cross-edges are added to the graph. Note that the use of a function hash is fundamental in findCrossEdges because it allows us to query in $O(1)$ time if a function in the set of added functions is present in any candidate parent.

\subsubsection{Complexity Analysis}

Let the set of malware samples be $S=\left\{s_{1}, \cdots, s_{n}\right\}$ and $\mathcal{F}$ be the set of functions appearing in all malware samples in $S$. Let $F: S \rightarrow 2^{\mathcal{F}}$ label each malware with the set of functions appearing in it (e.g., $F\left(s_{2}\right)=\left\{f_{5}, f_{8}\right\}$ means that functions $f_{5}$ and $f_{8}$ appear in $s_{2}$ ). Our algorithm is divided into three phases.

Phase I (clustering): The goal of this phase is to find a coarsest partition $\mathcal{P}=$ $\left\{G_{1}, G_{2}, \cdots, G_{k}\right\}$ of $S$ such that $s_{i}$ and $s_{j}$ are in the same group iff $F\left(s_{i}\right)=F\left(s_{j}\right)$. 
Using hashing, the cost of finding this partition is $O(n)$. We overload the function $F$, and use $F\left(G_{i}\right)$ to be $F(s)$ for some $s \in G_{i}$ (since all $s \in G\left(s_{i}\right)$ have the same label $F(s)$, picking an arbitrary $s$ does not create an issue.)

Phase II (Lineage Tree): This tree $T=(V, E)$ has $k$ vertices $\{1, \cdots, k\}$, where vertex $i$ corresponds to group $G_{i}$. Let inst $\left(s_{i}, s_{j}\right)$ be the number of instructions common between $s_{i}$ and $s_{j}$. We say that $G_{i} \prec G_{j}$ iff $F(i) \subset F(j)$. For each $j \in V$, define $\operatorname{dist}(i, j)$ (for $i \in V$ ) to be the 2-tuple $\left\langle\left|F\left(G_{i}\right) \cup F\left(G_{j}\right)\right|\right.$, inst $\left.\left(s_{i}, s_{j}\right)\right\rangle$. We say that $\langle n, m\rangle<\left\langle n^{\prime}, m^{\prime}\right\rangle$ iff $n<n^{\prime}$ or $\left(n=n^{\prime} \wedge m<m^{\prime}\right)$ (i.e., we use lexicographical ordering on the 2-tuple). Let $\operatorname{MIN}(j) \subseteq V-\{j\}$ be all the minimal elements according to the metric dist (i.e., $i \in \operatorname{MIN}(j)$ iff there does no exist a $k \in V-\{j\}$ such that $\operatorname{dist}(k, j)<\operatorname{dist}(i, j))$. We add an edge $(i, j) \in E$ where $i$ is the highest index in the set $\operatorname{MIN}(j)$. Assuming that all the metrics have been pre-computed, computing edges in $E$ takes at most $O\left(k^{2}\right)$ time.

Phase III (Adding cross-edges): Next we add cross-edges to the tree $T=(V, E)$ constructed in phase II. Let the set of cross-edges $E_{c} \subseteq V \times V$. Let $F_{T}: V \rightarrow \mathcal{F}$ be a such that $F_{T}(i)$ is $F(i)$ minus the labels of the parent of $i$ and children of $i$ (if the parent or children of $i$ don't exist, then we do subtract anything from $F(i)$ ). For each $j \in V$, let $\mathrm{Z}(j)$ be all $i \in V$ that satisfy the following conditions: $i \neq j$ and $i$ is not the parent or child of $j$ in the lineage tree $T$. We want to find a set of indices of minimal size $I(j) \subseteq \mathrm{Z}(j)$, such that $F_{T}(j) \subseteq \bigcup_{i \in I(j)} F(i)$ (in other words we want to find the minimal cover for the set $F_{t}(j)$ using sets whose indices are in $\mathrm{Z}(j)$. Finding the minimal cover is NP-hard, so we rely on iterative algorithm to "approximate" $I(j)$. We add $I(j) \times\{j\}$ to the set of cross-edges $E_{c}$. Our iterative algorithm to pick $I(j)$ takes time $O(k)$ for each $j$. Therefore, the entire running time of this phase is $O\left(k^{2}\right)$.

\subsection{Evaluation on Benign Programs}

In this section we evaluate the accuracy of our approach on benign open-source programs for which we have ground truth. Section 4.7.1 presents the accuracy metrics, Section 4.7.2 the unpacking and disassembly results, and Section 4.7.3 the lineage inference results. The evaluation on malware is in Section 4.8.

\subsubsection{Accuracy Metrics}

No prior unpacking approach proposes metrics to scientifically quantify the quality of the unpacking. Quoting a recent unpacking work: "we recognize that we have not been able to define a metric that allow us to adequately determine code coverage" [10]. We propose to evaluate the accuracy of the unpacking and disassembly process by measuring how similar the original program (before packing) is to its unpacked representation. For this, we compare the IDA database of the original program, disassembled 
Chapter 4. Lineage Inference of Packed Malware

\begin{tabular}{|l|l|r|rrr|rrr|}
\hline \multirow{2}{*}{ Packer } & \multirow{2}{*}{ Version } & \multirow{2}{*}{$|P|$} & \multicolumn{3}{|c|}{ FC } & \multicolumn{3}{c|}{ FNR } \\
\cline { 4 - 8 } & & & Min & Avg & Max & Min & Avg & Max \\
\hline ACProtect & 2.0 & 21 & $48.68 \%$ & $68.73 \%$ & $79.79 \%$ & $23.74 \%$ & $39.57 \%$ & $76.00 \%$ \\
\hline Armadillo & 8.20 & 2 & $61.22 \%$ & $61.74 \%$ & $62.26 \%$ & $32.65 \%$ & $35.71 \%$ & $38.78 \%$ \\
\hline ASPack & 2.12 & 21 & $46.91 \%$ & $70.48 \%$ & $81.53 \%$ & $16.62 \%$ & $24.18 \%$ & $40.35 \%$ \\
\hline eXPressor & 1.8 .0 .1 & 21 & $47.01 \%$ & $70.58 \%$ & $81.53 \%$ & $16.74 \%$ & $24.79 \%$ & $43.33 \%$ \\
\hline FSG & 2.0 & 21 & $46.91 \%$ & $70.48 \%$ & $81.53 \%$ & $15.69 \%$ & $21.49 \%$ & $30.73 \%$ \\
\hline MEW 11 SE & 1.2 & 21 & $46.91 \%$ & $70.48 \%$ & $81.53 \%$ & $16.47 \%$ & $22.60 \%$ & $31.14 \%$ \\
\hline MoleBox & 2.5 .13 & 21 & $48.78 \%$ & $68.63 \%$ & $79.79 \%$ & $27.87 \%$ & $46.28 \%$ & $86.55 \%$ \\
\hline MPRESS & 2.19 & 21 & $49.14 \%$ & $69.46 \%$ & $80.14 \%$ & $15.23 \%$ & $22.24 \%$ & $33.33 \%$ \\
\hline Packman & 1.0 & 21 & $46.91 \%$ & $70.48 \%$ & $81.53 \%$ & $15.84 \%$ & $21.77 \%$ & $30.87 \%$ \\
\hline PECompact & 1.71 & 21 & $46.91 \%$ & $70.48 \%$ & $81.53 \%$ & $16.08 \%$ & $22.58 \%$ & $33.33 \%$ \\
\hline PELock & 2.04 & 21 & $48.78 \%$ & $69.25 \%$ & $79.79 \%$ & $25.81 \%$ & $36.61 \%$ & $47.73 \%$ \\
\hline PESpin & 1.33 & 21 & $48.68 \%$ & $67.49 \%$ & $79.44 \%$ & $20.26 \%$ & $29.25 \%$ & $55.74 \%$ \\
\hline Petite & 2.4 & 21 & $47.01 \%$ & $70.48 \%$ & $81.53 \%$ & $15.97 \%$ & $22.28 \%$ & $32.00 \%$ \\
\hline RLPack & 1.21 & 21 & $48.62 \%$ & $69.10 \%$ & $79.79 \%$ & $17.02 \%$ & $24.55 \%$ & $39.29 \%$ \\
\hline UPX & 3.91 & 21 & $47.01 \%$ & $70.48 \%$ & $81.53 \%$ & $15.66 \%$ & $21.16 \%$ & $30.73 \%$ \\
\hline WinUPack & 0.39 & 21 & $46.91 \%$ & $70.48 \%$ & $81.53 \%$ & $15.69 \%$ & $21.39 \%$ & $30.73 \%$ \\
\hline YodaCrypter & 1.3 & 20 & $48.68 \%$ & $69.14 \%$ & $79.79 \%$ & $16.79 \%$ & $24.31 \%$ & $41.18 \%$ \\
\hline YodaProtector & 1.03 & 21 & $48.68 \%$ & $68.74 \%$ & $79.79 \%$ & $22.37 \%$ & $34.69 \%$ & $71.96 \%$ \\
\hline \hline All & & 21 & $46.91 \%$ & $69.26 \%$ & $81.53 \%$ & $15.23 \%$ & $27.53 \%$ & $86.55 \%$ \\
\hline
\end{tabular}

Table 4.1: Unpacking and disassembly accuracy evaluation on all SPEC CPU 2006 C programs with SPP-NOP, each packed with 18 packers. For each packer it shows the number of programs evaluated, function coverage, and function noise ratio.

using symbol information to prevent errors, with the IDA database output after packing the program, and processing the packed program with the unpacking and disassembly modules.

We propose two new metrics that separately quantify the amount of original code recovered and the amount of noise. We denote the set of functions in the original program database $F_{o}$ and in the unpacked program database $F_{u}$. We denote the set of correctly unpacked original functions as $F_{u}^{o} \subseteq F_{u}$. Correctly unpacked functions are the unpacked functions also present in the original code, i.e., $F_{u}^{o}=F_{u} \cap F_{o}$. Intuitively, unpacked functions that are not original code can be considered noise, i.e., $F_{u}^{n}=F_{u} \backslash F_{u}^{o}$. This noise includes, among others, unpacking functions and falsely identified functions during disassembly. Given the original and noise functions in the unpacked output, we define two metrics to evaluate accuracy: function coverage (FC) and function noise ratio (FNR).

Function coverage. The fraction of original functions the unpacking recovers over the number of functions in the original program. Ranges from zero (no original functions recovered) to one (all original functions recovered):

$$
F C=\frac{\left|F_{u}^{o}\right|}{\left|F_{o}\right|}=\frac{\left|F_{u} \cap F_{o}\right|}{\left|F_{o}\right|}
$$

Function noise ratio. The fraction of noise functions over the total number of functions in the unpacked representation of the program. Ranges from zero (no noise) to one (all noise, no original functions recovered): 


$$
F N R=\frac{\left|F_{u}^{n}\right|}{\left|F_{u}\right|}=\frac{\left|F_{u} \backslash F_{u}^{o}\right|}{\left|F_{u}\right|}
$$

When computing FC and FNR we identify a function by its SPP-NOP hash, and we ignore short functions, i.e., those with at most two instructions, since those can cause spurious matches.

\subsubsection{Unpacking \& Disassembly Accuracy}

We evaluate the accuracy of the unpacking and disassembly modules using a dataset of 21 programs for which we have the source code: $19 \mathrm{C}$ programs from the SPEC CPU 2006 benchmark [173] and $2 \mathrm{C}++$ programs from the Olden benchmark [174]. We compile them using Visual Studio with optimization level -O2 and debugging symbols, producing an executable and a PDB symbols file. We use IDA to disassemble them providing the PDB symbols file as input. Next, we pack the executables with 18 off-the-shelf packers and examine that they work. We find that the SPEC CPU 2006 programs do not work after packing with Armadillo, but the 2 Olden benchmark programs work. There is also one SPEC CPU 2006 program that does not work after being packed with YodaCrypter. We input the correctly packed executables to the unpacking and disassembly modules, which output an IDA database with the recovered code.

Table 4.1 summarizes the accuracy results using the metrics described in Section 4.7.1. Overall, our unpacking and disassembly modules achieve an average function coverage of $69 \%$ and an average function noise ratio of $27 \%$. Manual analysis of the results shows that: (a) all original functions are present in the differential memory snapshots and thus in the unpacked IDA database, (b) the same functions are missed in a program regardless of the packer, (c) the disassembly process fails to identify always the start of the same functions, and (d) the vast majority of additional functions (i.e., noise) are added by the packing process to perform the unpacking at runtime. Thus, all the original code is present in the differential memory snapshots output by the unpacking, but, regardless of the packer, the disassembly process fails to identify the entry point of the same functions. Next, we analyze this issue in more detail using the BHBmk program from the Olden benchmark. We observe the same causes in other programs.

For BHBmk, the analysis reveals that ByteWeight only finds 37 original functions (i.e., misses 15 functions) despite all functions being present in the differential memory snapshots provided as input to ByteWeight. Manual analysis on the missed functions shows two reasons. First, 9 functions are wrappers to other functions. These wrappers start with a sequence of push instructions followed by a call to the wrapped function, which is a common sequence in the middle of functions and thus not detected by ByteWeight as a function entry point. Second, 6 functions have uncommon preambles not in the ByteWeight model. Note that even if ByteWeight misses a function start, IDA may still identify the function if there is a direct call to it. Of the 15 BHBmk functions missed by ByteWeight, IDA identifies 10 this way. There are also 4 functions whose 


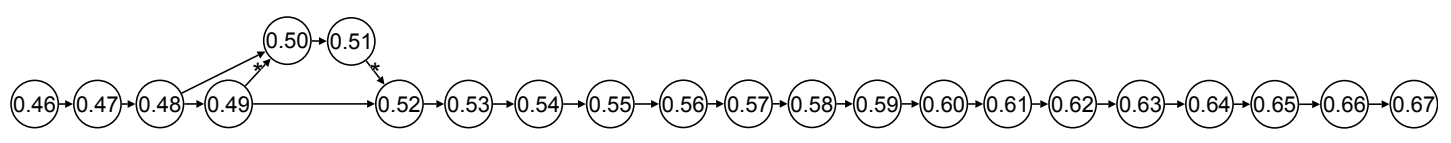

Figure 4.3: PUTTY lineage graph. Cross-edges are marked with asterisk.

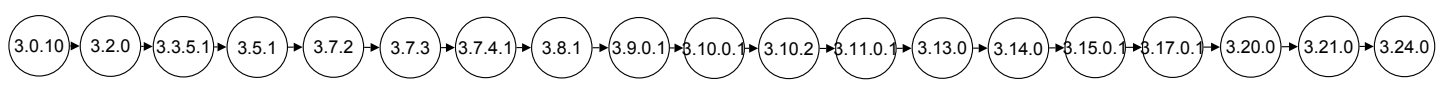

Figure 4.4: FZPUTTYGEN lineage graph.

start address ByteWeight finds, but IDA refuses to create a function at that address. There are two reasons for this. First, IDA may run into a disassembly error inside the function failing to find the function's boundary. Second, IDA may have already marked that code as belonging to another function and refuses to create a new function there.

To conclude, the unpacking module properly outputs all original code into the differential memory snapshots, but not all original functions would be available to the lineage inference because the disassembly misses some functions. Most of the missed functions are due to ByteWeight and we plan to either improve it or replace it in our next release. Most of the additional functions (i.e., noise) implement the unpacking process and we plan to identify those as a next step.

\subsubsection{Lineage Inference Accuracy}

To evaluate the lineage inference we use 631 release versions of 13 open-source Windows programs. Seven of the programs are part of the PUTTY distribution [175] (PutTy, PutTygen, PutTytel, PSFtP, PSCP, Plink, Pageant), three are part of FileZilla [176] (FileZILla, FZPUTTYGEN, FZSFTP), and the other three are WinSCP [177], NotePAD++ [178], and PROCESSHACKER [179]. We are interested in evaluating the lineage inference as a stand-alone module. Thus, we evaluate on the released (i.e., unpacked) executables. To evaluate a program, we generate an IDA database for the executable of each version and input these IDA databases to the lineage inference module to produce the lineage graph. Then, we compare the lineage graph to the expected lineage graph built using the version numbers.

We manually identify the number of binary versions among the input releases. There are three main reasons why consecutive source releases may not create a new binary version. First, some versions do not modify the source code, but only auxiliary files (e.g., configuration files, images). This happens, for example, between FILEZILLA 3.9.0 and 3.9.0.1. Second, some versions perform source code modifications that do not affect the final executable, e.g., variable renaming. Third, in distributions with multiple programs, source code changes may affect some programs, but not others. For example, the changes between 0.61 and 0.62 affected PUTTY and PUTTYtel, but not PuTTYgen. Thus, PuTTYGen 0.61 and 0.62 are the same binary 


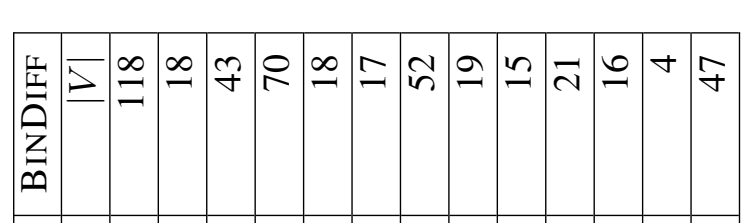

உோ

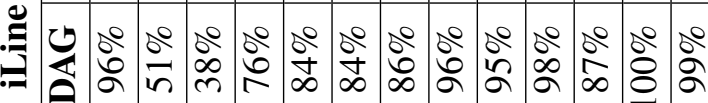

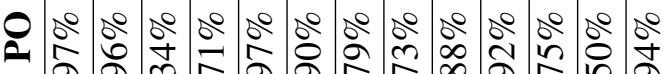

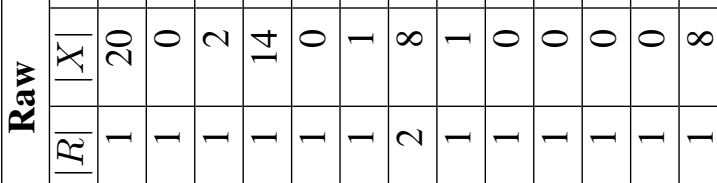

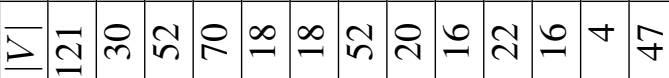

ஃ

言

जิ

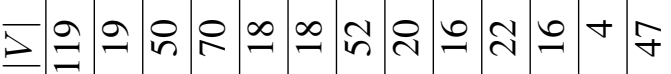

크ำ으ำำำ

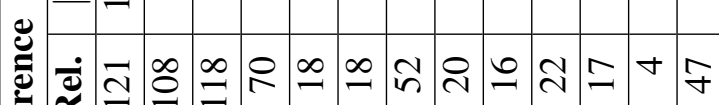

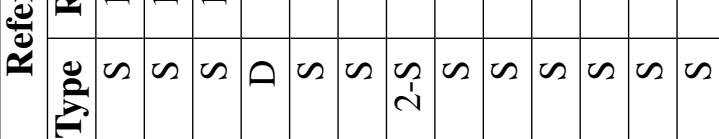

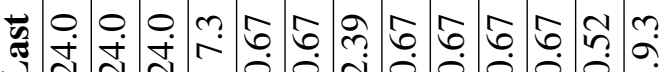

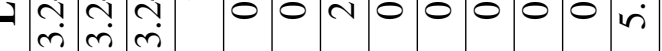

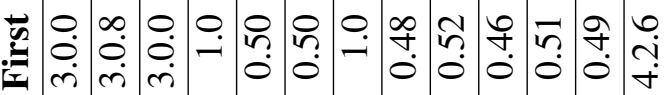

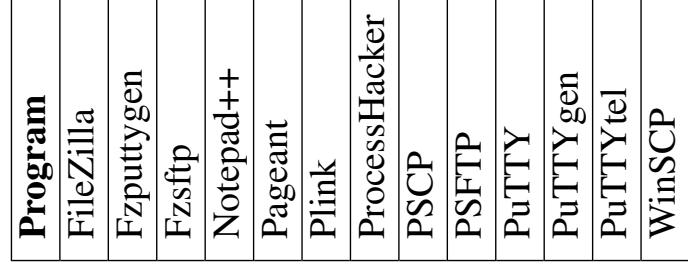

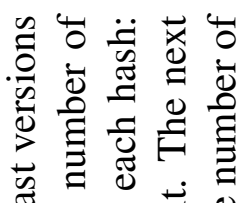

悉

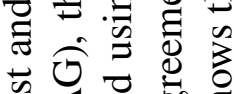

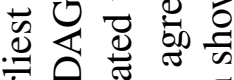

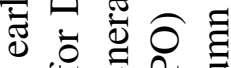

\& 0 品

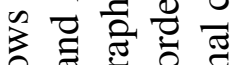

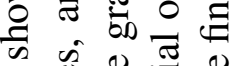

क 仓

$\exists: \Xi \approx$

$=\dot{z} \Xi \dot{\Xi}$

हี่

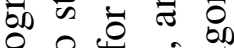

ํㅠ

ฮั

- $n$. क 0

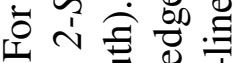

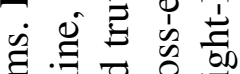

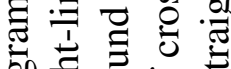

क्षे

范

ष क

亏ั

i $\approx$. 0 क

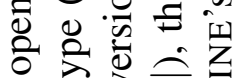

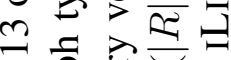

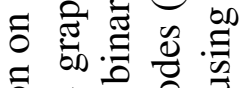

.

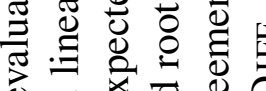

己े

ए

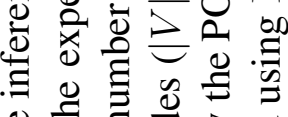

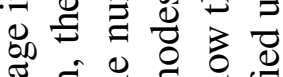

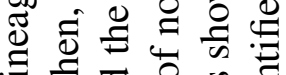

Э

ஸे

츄 ङ

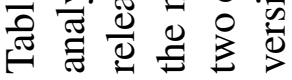


version.

Table 4.2 summarizes the results. For each program it shows the first and last versions analyzed. Then, it shows the expected lineage graph type, the number of releases, and the number of binary versions (i.e., ground truth). The type can be $S$ for straight-line, 2-S for two straight lines, and $D$ for branching and merging, i.e., DAG. Next, it shows for the lineage graph generated using each hash: the number of nodes and root nodes, the number of cross-edges, and the partial order agreement, a measure of lineage graph accuracy proposed in ILINE [15]. Finally, it shows the PO agreement using ILINE's DAG and straight-line algorithms.

The results for Phase I show that the versions identified by the SPP-NOP hash match the expected binary versions for all 13 programs, while the raw hash identifies the correct binary versions for 9 programs. For the 3 FilEZILLA programs, the number of nodes identified by the SPP-NOP and raw hashes differs, with the raw hash identifying a larger number of binary versions. In all cases, when a node in the lineage graph represents multiple releases (each with a different executable hash), those releases are consecutive. These results indicate that the SPP-NOP hash is better at identifying versions than the raw hash.

Analysis of the phase II results shows that the generated lineage tree is more accurate with the SPP-NOP hash in 9 programs and has the same accuracy with both hashes in 4 programs. Thus, the SPP-NOP hash works better than the raw hash also in this phase. The most common error is that a version $x$ is more similar to version $x+2$ than to version $x+1$. These errors happen in all but one program and manifest in two cases: two consecutive versions swap their positions or a version has two successors one creating a branch with a single node and no merging point. An example of the first case is PUTTYGEN where version 0.58 is followed by 0.60 , which is followed by 0.59. An example of the second case is FZSFTP version 3.0.6, which is followed by both 3.0.7.1 and 3.0.8.1. The latter is followed by all other versions, while 3.0.7.1 is in a branch by itself. Another error affecting 2 programs is that the wrong root is chosen, i.e., the chosen root is not the earliest version, but the second earliest.

All programs have the correct number of roots, one for straight line or DAG development, and two for PROCESSHACKER that has two separate lines. For the 12 programs with straight-line (or 2-straight) development, phase III can only introduce errors as there is no branching and merging. But, our algorithm should identify those cases and avoid inserting cross-edges. The results show that cross-edges are inserted for 7 programs. In 5 of those programs only 1 or 2 cross-edges are inserted and these typically are inserted where there was an error in Phase II. For example, Figure 4.3 shows the final lineage graph for PUTTY. Version 0.48 is followed by versions 0.49 and 0.50 after Phase II, and phase III inserts cross-edges between 0.49 and 0.50 and between 0.51 and 0.52 . If 0.50 had followed 0.49 after Phase II, no cross-edge would have been inserted as 0.49 would be a predecessor of 0.50 and thus not included in the list of candidate parents. The same applies to the cross-edge from 0.51 to 0.52 . Figure 4.4 shows the final lineage graph for FZPUTTYGEN. In this case, the lineage graph has no errors and perfectly matches its straight-line development. 


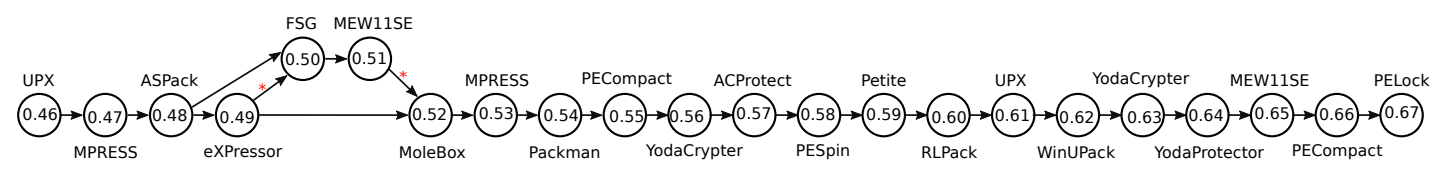

Figure 4.5: Lineage graph for PUTTY when each version is packed with a different packer.

Comparison with ILINE. We compare our lineage inference algorithm with ILINE, the current state-of-the-art lineage inference approach. ILINE proposes two different lineage inference algorithms for straight-line code and branching and merging, and assumes the analyst knows which of the algorithms should be used (or which results to trust if both algorithms are used). Since ILINE is not publicly available we have reimplemented both of its algorithms. The two rightmost columns in Table 4.2 show the results for ILINE's DAG and straight-line (SL) algorithms using as symmetric distance the number of differing functions between both samples according to the SPP-NOP hash. This allows us to compare ILINE's algorithms with our algorithm using similar features. To measure accuracy, we use the partial ordering agreement (PO) metric proposed in ILINE. For PROCESSHACKER, we first split versions into both development lines and apply ILINE's SL algorithm in each line separately. Our lineage inference module using SPP-NOP achieves an average 95\% PO over the 13 programs, compared to $84 \%$ for ILINE's DAG and $93 \%$ for ILINE's straight line. Thus, our algorithm outperforms both of ILINE's algorithms despite using no apriori knowledge about the development model, which is fundamental when operating with malware. The best results for ILINE are obtained by assuming straight-line development, but this means that a wrong (straight-line) lineage graph would be produced for any program using branching and merging.

Comparison with BINDIFF. We compare our Phase I results with BINDIFF. Pairwise comparison of all versions does not finish in 8 hours for WINSCP and FILEZILLA. To address this, we first partition the executables by number of functions (i.e., executables with differing number of functions should be different versions) and only perform pairwise comparisons inside each partition. After aligning two executables with BINDIFF we consider they are the same version if BINDIFF successfully aligns all the functions in both executables and the similarity value for each function is over 0.9. The righmost column in Table 4.2 shows that in 7 out of 13 programs, this approach would introduce errors, i.e., incorrectly identifying similar versions as being the same. Furthermore, note that we cannot use BINDIFF in Phase III of our algorithm because it cannot check if a function appears in any of the predecessor nodes. We need function hashes for that.

Packed evaluation. To evaluate the lineage inference accuracy with packed executables, we perform the following experiment. We pack each version of PUTTY using a randomly selected packer and then apply the unpacking, disassembly, and lineage 
inference modules to build the lineage graph. This allows us to evaluate our lineage inference approach when different packers are used by the same program, as well as to check the impact on the lineage graph of any functions missed by the unpacking and disassembly modules. Figure 4.5 shows the produced lineage graph, which is identical to the unpacked version in Figure 4.3.

In summary, our evaluation has shown that: the SPP-NOP hash outperforms the raw hash both in node and edge identification; the lineage graphs produced using the SPP-NOP hash are more accurate than those produced by ILINE, without assuming a specific development model was used; our version identification improves on BINDIFF; and the lineage graphs are accurate despite the use of different packers.

\subsection{Evaluation on Malware}

This section evaluates our lineage approach on 10 malware families. We first describe our dataset, then in Section 4.8.1 we present the unpacking and disassembly results, and in Section 4.8.2 the lineage inference results.

Dataset. The PANDA team periodically records malware executions on a sandbox and makes these recordings publicly available [180]. The malware in those recordings is unlabeled, i.e., its family is unknown. To classify the malware, we first collect the AV labels for the samples using VirusTotal (VT) [151], an online service that analyzes files and URLs submitted by users. We use the AV labels as input to AVClass [181], an open-source malware labeling tool. AVClass outputs for each sample the most likely family name and a confidence factor based on the agreement across AV engines [165]. To compute the lineage graph we need a significant number of samples for the same family. Thus, we select 10 malware families for which more than 100 samples are labeled with high confidence by AVClass: AllaPle, IRCBOT, KLEZ, Loring, Memery, Picsys, Simbot, Sytro, Urelas, and VtFlooder. In total we evaluate on 7,793 malware samples. The largest number of family samples is 4,000 for ALLAPLE and the smallest 113 for MEMERY.

\subsubsection{Unpacking and Disassembly}

Table 4.3 details the unpacking and disassembly results for the 10 malware families. For each family, it shows: the number of executables analyzed; the number of instructions (in millions) traced for all malware processes; the number of malware processes traced; the number of waves for all malware processes; the unpacking runtime (in seconds); the total number of unpacked functions in the IDA databases (including short and external functions that IDA counts); and the disassembly runtime (in seconds).

For 6 families all samples have a single process, for LORING all samples have 2 processes, and the remaining three (i.e., IRCBOT, SIMBOT, URELAS) have samples with different number of processes. The number of waves ranges from 1 (i.e., not packed) up to 215 for a URELAS sample. Surprisingly, 7 families have a few samples 

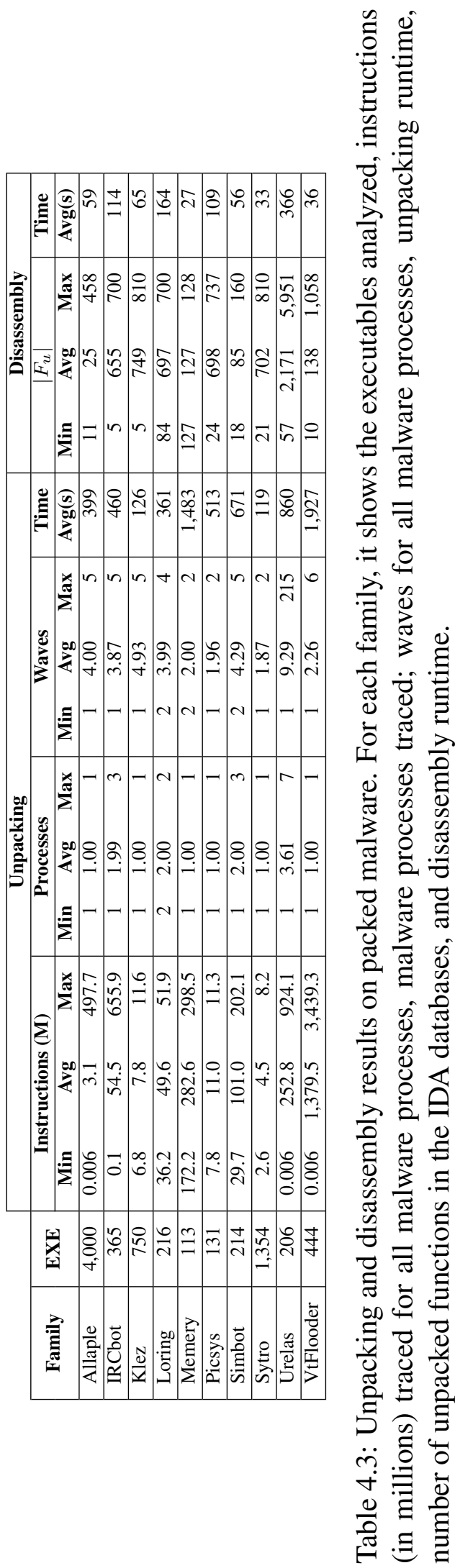


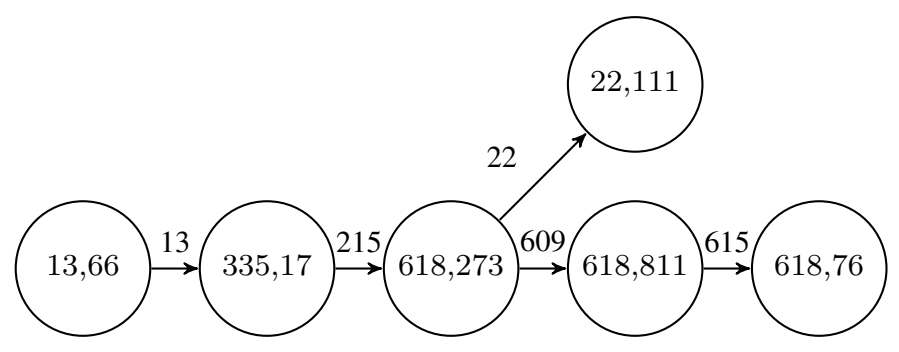

Figure 4.6: Lineage graph for SYTRO built using 1,354 samples. The node label indicates the number of functions in the version and the number of samples of that version. The edge label is the number of shared functions.

that are not packed (e.g., 5 out of 131 samples in PICSYS), which may be due to the developers forgetting to pack some samples or to early versions not being packed. On average, replaying a recording takes 11 minutes, the slowest being VTFLOODER with an average of 32 minutes per recording. The disassembly runtime takes on average 103 seconds per sample, of which $82 \%$ is due to the ByteWeight function identification.

\subsubsection{Lineage Inference}

Table 4.4 details the final lineage graphs output for the 10 malware families. The table shows the number of executables for each family and for each hash: the number of nodes and edges, the maximum number of samples in a version, the number of singleton versions representing only one sample, the maximum and minimum number of functions in a version, and the total number of functions across all versions.

We focus on the SPP-NOP hash results as Section 4.7.3 demonstrates that it outperforms the raw hash. The largest number of SPP-NOP versions is for URELAS with 123 and the smallest for LORING with 2. For 9 families, the number of versions using both hashes differs. Overall, we identify 293 SPP-NOP versions across the 10 families, compared to 7,793 input samples, a 26 times reduction (10x reduction for the raw hash). Thus, our lineage graph is a succinct summary of family evolution, achieving over an order of magnitude reduction in nodes compared to prior approaches where each input sample is a node.

The results show that some versions are more popular than others in terms of samples. For example, $99 \%$ of LORING and $92 \%$ of IRCBOT samples are derived from one version. On the other hand, 51\% of URELAS samples correspond to singleton versions, which may be due to a large amount of experimentation in the family, or to limited coverage of our input samples for some versions. The code of a family can significantly evolve over time. For example URELAS versions range from 42 up to 4,420 functions. We also observe different code base sizes across families. The most complicated malware families are URELAS and VTFLOODER with 7.7K and 5.3K functions across all versions, respectively. The simplest malware family is SIMBOT with a total of 126 functions in 216 samples. 


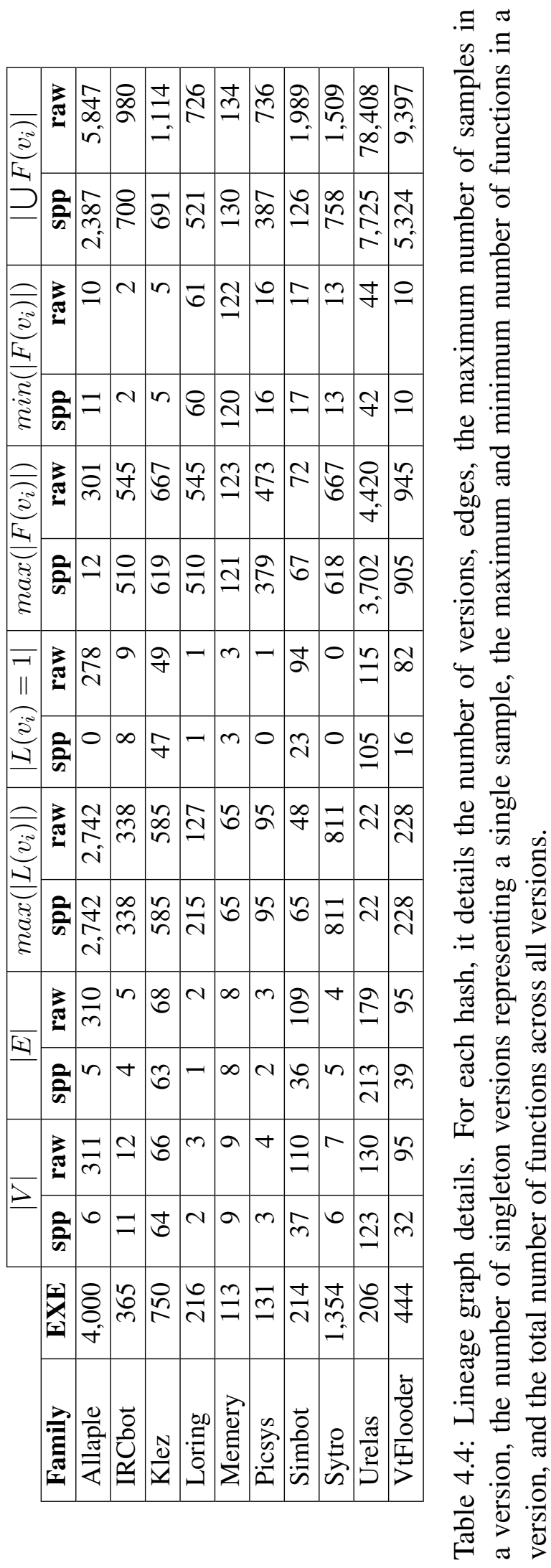


Of the 10 malware families, 3 have straight-line development (ALLAPLE, LORING, PICSYS). Figure 4.1 shows the final PICSYS lineage graph. MEMERY and SYTRO have mostly straight development with only one node having two branches, one of them leading to a single node, as illustrated in the lineage graph for SYTRO in Figure 4.6. SIMBOT has mostly straight development with 4 nodes having two branches. IRCBOT has multiple straight lines, although $98 \%$ of the input samples belong to the same line. Finally, the lineage graphs of 3 families (KLEZ, URELAS, VTFLOODER) are DAGs. These results show the variety of development models malware families may use and the difficulty of predicting the development model apriori.

To conclude the results show that the lineage graph is a succinct summary of the evolution of a malware family, which reduces the number of input samples to a much smaller (i.e., 26 times smaller) number of versions. The 10 malware families show varying development models that are captured by our lineage inference algorithm.

\subsection{Discussion}

This section discusses some limitations of our work and directions for future investigation.

Packers that modify the original code. Our unpacking module handles packers where the original code is recovered at runtime. However, packers may transform the original code, so that it is no longer present in the packed executable. For example, virtualization-based packers such as Themida and VMProtect convert assembly into bytecode interpreted by a VM. One approach to handle such packers is analyzing the executable code output by the interpreter. We leave the support of such packers as future work.

Evasion. Similar to other dynamic analysis approaches, our unpacking can be evaded by techniques that detect the presence of a VM or emulator. We ameliorate this problem by incorporating countermeasures for specific anti-VM checks, but our countermeasures are not complete. Interestingly, we observe that anti-VM checks are typically packed themselves to avoid their presence flagging the executable as malware. If the anti-VM checks are unpacked simultaneously with the original code, by the time the malware executes the anti-VM checks our unpacking has already captured the original code in a differential memory snapshot.

Function identification. Most disassembly errors in our evaluation are due to missed functions. Our system currently uses ByteWeight [52] for function identification, but could use other tools. We are currently evaluating Nucleus [55], which should improve on ByteWeight results, but have not finished integrating it into our toolchain yet. 


\section{Conclusions \& Future Work}

In this chapter, we briefly summarize the thesis and its contributions. We also discuss potential enhancements to our existing malware version identification and lineage inference approach and provide the future research directions.

\subsection{Conclusions}

In this thesis, we contributed an approach that automatically identified versions of a malware family and recovered its lineage as a lineage graph. Our approach used binary code similarity as a vehicle to solve the problem of malware version identification and lineage inference. This has motivated us to explore existing binary code similarity approaches and understand their capabilities and limitations.

In Chapter 3, we contributed a systematic survey of binary code similarity research, and systematized 59 works on four aspects: applications it enabled, approaches used to compare pieces of binary code, how the approaches were implemented, and benchmarks and evaluation methodologies. There are three additional takeaways from this chapter. First, the problem of binary code similarity has evolved over the past years. At the onset, binary code similarity was a problem of byte-level diffing of two arbitrary binary files (e.g., media, audio, executables). After that, it became a binary code diffing problem of two versions of the same program. Nowadays, it is a binary code search problem with additional constraints like the use of different compilers, compilation options, and CPU architectures. Second, the field of binary code similarity research has matured. There exists approaches that perform cross-architecture (e.g., x86 vs MIPS), cross-compiler (e.g., GCC vs VS), cross-optimization (e.g., O1 vs O3), and cross-OS (e.g., PE vs ELF) binary code comparison. Third, the research community has moved on from syntactical and structural comparison to embedding-based binary code comparison. The focus has shifted on learning the semantics of the underlying pieces of binary code as numerical embeddings with deep learning techniques such as neural networks and machine language translation. 
In Chapter 4, we presented our approach for inferring the lineage of packed malware automatically. This chapter addressed three subproblems of packed malware lineage inference: malware version identification; a lineage algorithm oblivious to malware development models; and accuracy metrics to evaluate the malware unpacking and disassembly. For malware versions identification, we devised a technique based on generic malware unpacking, static analysis, and function and program hashes. We used generic malware unpacking to recover the original binary code of a packed malware sample. Then, we employed static analysis to disassemble recovered binary code, i.e., function identification and instructions disassembly. Once a malware sample was unpacked, we used two types of hashes and a code indexing technique to identify shared functions among packed malware samples. Based on these hashes, we designed the first approach to identify the versions of a malware family. We tested our approach on 13 benign programs and compared its accuracy with BINDIFF. We also applied our approach to identify malware version of 10 popular malware families. On average, our approach resulted in $26 \times$ reduction from input samples to malware versions.

For inferring lineage of identified versions, we developed an algorithm based on the shared functions among the versions of the malware family. The proposed algorithm is oblivious to the malware development model (i.e., straight-line, k-straightlines, branching and merging). We evaluated the algorithm on 631 versions of 13 open-source programs and also compared with ILINE, a state-of-the-art lineage inference approach. Overall, our algorithm achieved a 95\% accuracy.

For measuring the effectiveness of malware unpacking and disassembly, we proposed two accuracy metrics: Function Coverage (FC) and Function to Noise Ratio (FNR). Our unpacking and disassembly module used ByteWeight [52] for function identification and IDA for disassembly. We measured FC and FNR of the unpacking module on 359 packed binary programs produced from 21 programs and 18 packers. This module recovered up to $81 \%$ of original binary code.

\subsection{Future Work}

Compilers \& compilation options. Our malware version identification approach use identical comparison to find malware samples with the same set of functions. However, different compilers and their compilation options may produce binary code with varying number of functions and instructions. For instance, function inlining can change the number of functions in a binary program. This could be problematic in our case as our approach will introduce more nodes in the lineage graph. Our approach has already provided a 26 times reduction from input samples to malware versions. This enhancement will further reduce the number of nodes in the lineage graph. Therefore, as future work, we would like to extend our approach to counter different compilers and compilation options.

Cross-architecture version identification. In this thesis, we have focused on Windows- 
based malware families targeting x86 and x86-64. However, recent years have witnessed a rise in Internet of Things (IoT) malware [7] that target IoT devices having different hardware platforms, e.g., ARM, MIPS, x86. It means, the malware authors compile the same source code for different hardware platforms, and those malware samples are same malware family version. Thus, recovering the lineage of an IoT malware family requires identifying cross-architecture malware versions. In the future, we would like to explore and extend our current malware lineage inference approach to IoT malware families.

Supporting other operating systems. Currently, our approach supports unpacking of malware samples for Microsoft Windows, i.e., PE executables on top of any CPU architectures. It does not support malware unpacking for other operating systems (e.g., Linux, MacOS) and executable file formats (e.g., ELF). In the future, we have plans to extend our unpacking module to support Linux and other operating systems. This updating can be carried out independently of the malware version identification and lineage inference approach as both are independent of each other.

Function labeling. Our malware unpacking and disassembly approach outputs two types of functions: original malware functions and noise functions (e.g., packer functions). Currently, we presume that an update of the packing routines (or packer functions) results in a new version of a malware family because security professionals may be interested in understanding the packing process and the new packing techniques. However, we could also consider all polymorphic variants of a malware sample as a single version regardless of the packing functions, i.e., version identification could be based only on the original binary code of a malware sample. In the future, we would like to label packers and original malware functions. This labelling would help to use only original malware code for version identification and lineage inference. Moreover, packer's code could be analyzed separately to develop packer detection techniques.

Summary of semantics changes. Our lineage inference approach enables comparing the functions added and removed across versions of a malware family. However, the analyst still needs to examine the added and removed functions to understand their functionality. In future work, we could provide the analyst with summaries of the semantics of those functions to further reduce the malware analysis effort.

Robustness against obfuscation. We leveraged SPP-NOP to compare functions. SPPNOP is robust against few binary code obfuscations like instructions reordering, basic blocks reordering, extra nop instructions, dead code. However, many obfuscation techniques can circumvent SPP-NOP such as semantically equivalent instructions. As a future extension, we would like to study and update our approach to be robust against advance obfuscations. 


\section{Funding Acknowledgments}

This research was partially supported by the European Union Horizon 2020 ElasTest (ICT-10-2016-731535) project; by the Regional Government of Madrid through the BLOQUES-CM (S2018/TCS-4339) and N-GREENS Software-CM (S2013/ICE-2731) projects; and by the Spanish Government through the MINECO SCUM (RTI2018102043-B-I00), DEDETIS (TIN2015-7013-R), and StrongSoft (TIN2012-39391-C0401) projects. This research is also supported by the EIE Funds of the European Union. 
Table 5.1: Comparison of the binary analysis platforms used in the implementation of binary code similarity approaches. This table overlaps with previous work on type inference [1]. We have added following six platforms: Angr, Beaengine, Cuckoo, NewBasic, McSema, Vulcan.

\begin{tabular}{|c|c|c|c|c|c|c|c|c|c|c|c|c|c|c|}
\hline \multirow[b]{2}{*}{ Platform } & \multirow[b]{2}{*}{ IR } & \multirow[b]{2}{*}{ 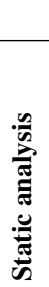 } & \multirow[b]{2}{*}{ 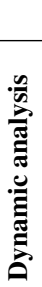 } & \multicolumn{8}{|c|}{ Target Arch. \& OS } & \multicolumn{3}{|c|}{ Release } \\
\hline & & & & $\underset{x}{\infty}$ & $\begin{array}{l}\text { J } \\
\dot{d} \\
\infty \\
x\end{array}$ & $\underset{\&}{*}$ & $\underline{\Sigma}$ & 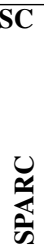 & $\begin{array}{l}0 \\
0 \\
0 \\
0 \\
0 \\
0\end{array}$ & $\frac{\sqrt[c]{2}}{2}$ & 四 & 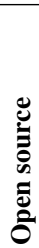 & 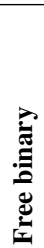 & 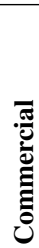 \\
\hline Angr [182] & VEX & $\checkmark$ & $x$ & $\checkmark$ & $x$ & $\checkmark$ & $\checkmark$ & $x$ & $x$ & $\checkmark$ & $\checkmark$ & $\checkmark$ & $x$ & $x$ \\
\hline BAP [183] & BIL & $\checkmark$ & $\checkmark$ & $\checkmark$ & $\checkmark$ & $\checkmark$ & $x$ & $x$ & $x$ & $\checkmark$ & $\checkmark$ & $\checkmark$ & $x$ & $x$ \\
\hline Beaengine [184] & $x$ & $\checkmark$ & $x$ & $\checkmark$ & $x$ & $x$ & $x$ & $x$ & $x$ & $\checkmark$ & $x$ & $\checkmark$ & $x$ & $x$ \\
\hline BitBlaze [185] & VINE & $\checkmark$ & $\checkmark$ & $\checkmark$ & $x$ & $x$ & $x$ & $x$ & $x$ & $\checkmark$ & $\checkmark$ & $\checkmark$ & $x$ & $x$ \\
\hline Boomerang [186] & RTL & $\checkmark$ & $x$ & $\checkmark$ & $x$ & $x$ & $\checkmark$ & $\checkmark$ & $\checkmark$ & $\checkmark$ & $\checkmark$ & $\checkmark$ & $x$ & $x$ \\
\hline Cuckoo [187] & $x$ & $x$ & $\checkmark$ & $\checkmark$ & $\checkmark$ & $\checkmark$ & $x$ & $x$ & $x$ & $\checkmark$ & $\checkmark$ & $\checkmark$ & $x$ & $x$ \\
\hline Dyninst [188] & $x$ & $\checkmark$ & $\checkmark$ & $\checkmark$ & $\checkmark$ & $x$ & $x$ & $x$ & $x$ & $x$ & $\checkmark$ & $\checkmark$ & $x$ & $x$ \\
\hline IDA [163] & IDA & $\checkmark$ & $x$ & $\checkmark$ & $\checkmark$ & $\checkmark$ & $\checkmark$ & $\checkmark$ & $\checkmark$ & $\checkmark$ & $\checkmark$ & $x$ & $x$ & $\checkmark$ \\
\hline LLVM [189] & LLVM-IR & $\checkmark$ & $x$ & $x$ & $x$ & $x$ & $x$ & $x$ & $x$ & $x$ & $x$ & $\checkmark$ & $x$ & $x$ \\
\hline NewBasic [190] & $x$ & $\checkmark$ & $x$ & $\checkmark$ & $x$ & $x$ & $x$ & $x$ & $x$ & $x$ & $x$ & $\checkmark$ & $x$ & $x$ \\
\hline McSema [191] & LLVM-IR & $\checkmark$ & $x$ & $\checkmark$ & $\checkmark$ & $\checkmark$ & $x$ & $x$ & $x$ & $\checkmark$ & $\checkmark$ & $\checkmark$ & $x$ & $x$ \\
\hline PIN [192] & $x$ & $x$ & $\checkmark$ & $\checkmark$ & $\checkmark$ & $x$ & $x$ & $x$ & $x$ & $\checkmark$ & $\checkmark$ & $x$ & $\checkmark$ & $x$ \\
\hline QEMU [193] & TCG & $x$ & $\checkmark$ & $\checkmark$ & $\checkmark$ & $\checkmark$ & $\checkmark$ & $\checkmark$ & $\checkmark$ & $\checkmark$ & $\checkmark$ & $\checkmark$ & $x$ & $x$ \\
\hline ROSE [194] & SAGE-III & $\checkmark$ & $x$ & $\checkmark$ & $x$ & $\checkmark$ & $\checkmark$ & $\checkmark$ & $\checkmark$ & $\checkmark$ & $\checkmark$ & $\checkmark$ & $x$ & $x$ \\
\hline Valgrind [195] & VEX & $x$ & $\checkmark$ & $\checkmark$ & $\checkmark$ & $\checkmark$ & $\checkmark$ & $\checkmark$ & $\checkmark$ & $\checkmark$ & $\checkmark$ & $\checkmark$ & $x$ & $x$ \\
\hline Vulcan [196] & VULCAN-IR & $\checkmark$ & $x$ & $\checkmark$ & $\checkmark$ & $x$ & $x$ & $x$ & $x$ & $\checkmark$ & $x$ & $x$ & $x$ & $x$ \\
\hline
\end{tabular}

Implmentation platforms. Table 5.1 shows the intermediate representation (IR) used by the platform, whether it supports static and dynamic analysis, the target architectures and operating systems it supports, and how it is released (open source or free binary). Among the 17 platforms, 12 support static analysis and 7 dynamic analysis. The functionality provided by static analysis platforms widely varies. IDA is a disassem- 
bler, Boomerang is a decompiler, and the rest offer diverse static analysis functionality such as disassembly, building control flow graphs and call graphs, IR simplifications, and data flow propagation. All dynamic analysis platforms can run unmodified binaries (i.e., no need to recompile or relink). QEMU is a whole system emulator that can run a full guest OS (e.g., Windows) on a potentially different host OS (e.g., Linux). Dyninst, PIN, and Valgrind execute an unmodified target program with customizable instrumentation (e.g., through binary rewriting) on the CPU of the host system. BAP and BITBLAZE build their dynamic analysis components on top of PIN and QEMU. 


\section{Bibliography}

[1] J. Caballero and Z. Lin, "Type Inference on Executables," ACM Computing Surveys, vol. 48, pp. 1-35, May 2016.

[2] A. Calleja, J. Tapiador, and J. Caballero, "A Look into 30 Years of Malware Development from a Software Metrics Perspective," in Proceedings of the 19th International Symposium on Research in Attacks, Intrusions and Defenses, (Evry, France), September 2016.

[3] A. Calleja, J. Tapiador, and J. Caballero, "The MalSource Dataset: Quantifying Complexity and Code Reuse in Malware Development," IEEE Transactions on Information Forensics and Security, 2019.

[4] "Symantec Internet Security Threat Report, Volumn 23," 2018. https://www.symantec.com/content/dam/symantec/docs / reports/istr-23-2018-en.pdf.

[5] "Quarterly Threat Landscape Report, 3rd Quarter," 2018. https: //www.fortinet.com/content/dam/fortinet/assets / threat-reports/threat-report-q3-2018.pdf.

[6] "VirusTotal Submission Statistics of Last 7 Days," 2019. https://www. virustotal.com/en/statistics/.

[7] "Symantec Internet Security Threat Report, Volumn 24," 2019. https://www.symantec.com/content/dam/symantec/docs/ reports/istr-24-2019-en.pdf.

[8] J. Ma, J. Dunagan, H. J. Wang, S. Savage, and G. M. Voelker, "Finding Diversity in Remote Code Injection Exploits," in ACM Internet Measurement Conference, 2006. 
[9] X. Ugarte-Pedrero, D. Balzarotti, I. Santos, and P. G. Bringas, "SOK: Deep packer inspection: A longitudinal study of the complexity of run-time packers," in IEEE Symposium on Security and Privacy, 2015.

[10] G. Bonfante, J. Fernandez, J.-Y. Marion, B. Rouxel, F. Sabatier, and A. Thierry, "CoDisasm: Medium scale concatic disassembly of self-modifying binaries with overlapping instructions," in ACM Conference on Computer and Communications Security, 2015.

[11] X. Ugarte Pedrero, D. Balzarotti, I. Santos, and P. G. Bringas, "RAMBO: Runtime packer analysis with multiple branch observation," in Detection of Intrusions and Malware \& Vulnerability Assessment, 2016.

[12] M. G. Kang, P. Poosankam, and H. Yin, "Renovo: A Hidden Code Extractor for Packed Executables," in ACM Workshop on Recurring Malcode, 2007.

[13] L. Martignoni, M. Christodorescu, and S. Jha, "OmniUnpack: Fast, Generic, and Safe Unpacking of Malware," in Annual Computer Security Applications Conference, 2007.

[14] M. Lindorfer, A. Di Federico, F. Maggi, P. M. Comparetti, and S. Zanero, "Lines of Malicious Code: Insights into the Malicious Software Industry," in Annual Computer Security Applications Conference, 2012.

[15] J. Jang, M. Woo, and D. Brumley, "Towards Automatic Software Lineage Inference," in 22nd USENIX Security Symposium, 2013.

[16] G. B. Sorkin, "Grouping Related Computer Viruses into Families," Proceedings of the IBM Security ITS, 1994.

[17] D. B. Hull, "Computer viruses: naming and classification, part II," in Virus Bulletin Conference, October 1995.

[18] L. A. Goldberg, P. W. Goldberg, C. A. Phillips, and G. B. Sorkin, "Constructing computer virus phylogenies," in Annual Symposium on Combinatorial Pattern Matching, 1996.

[19] E. Carrera and G. Erdelyi, "Digital Genome Mapping - Advanced Binary Malware Analysis," in Virus Bulletin Conference, 2004.

[20] M. E. Karim, A. Walenstein, A. Lakhotia, and L. Parida, "Malware Phylogeny Generation Using Permutations of Code," Journal of Computer Virology, vol. 1, no. 1-2, 2005.

[21] S. Wehner, "Analyzing Worms and Network Traffic using Compression," Journal of Computer Security, vol. 15, no. 3, pp. 303-320, 2007. 
[22] A. Gupta, P. Kuppili, A. Akella, and P. Barford, "An Empirical Study of Malware Evolution," in International Conference on Communication Systems and Networks, 2009.

[23] W. M. Khoo and P. Lió, "Unity in Diversity: Phylogenetic-Inspired Techniques for Reverse Engineering and Detection of Malware Families," in SysSec Workshop, 2011.

[24] C. Darmetko, S. Jilcott, and J. Everett, "Inferring Accurate Histories of Malware Evolution from Structural Evidence," in Florida Artificial Intelligence Research Society Conference, 2013.

[25] "UPX: the Ultimate Packer for eXecutables," 2017. https://upx. github.io/.

[26] H. Flake, "Structural comparison of executable objects," in Detection of Intrusions and Malware \& Vulnerability Assessment, 2004.

[27] "PECompact Windows (PE) Executable Compressor," 2019. https:// bitsum.com/portfolio/pecompact/.

[28] "Win32 Executable Compressor," 2019. https://www.softpedia. com/get/Programming/Other-Programming-Files/Petite. shtml/.

[29] M. Christodorescu, S. Jha, and S. Katzenbeisser, "Malware Normalization," tech. rep., University of Wisconsin, Madison, WI, USA, November 2005.

[30] P. Royal, M. Halpin, D. Dagon, R. Edmonds, and W. Lee, "PolyUnpack: Automating the Hidden-Code Extraction of Unpack-Executing Malware," in Annual Computer Security Applications Conference, 2006.

[31] M. Lindorfer, A. Di Federico, F. Maggi, P. M. Comparetti, and S. Zanero, "Lines of Malicious Code: Insights into the Malicious Software Industry," in Annual Computer Security Applications Conference, ACM, 2012.

[32] M. Hayes, A. Walenstein, and A. Lakhotia, "Evaluation of Malware Phylogeny Modelling Systems Using Automated Variant Generation," Journal in Computer Virology, vol. 5, no. 4, pp. 335-343, 2009.

[33] T. Dumitras and I. Neamtiu, "Experimental Challenges in Cybersecurity: a Story of Provenance and Lineage for Malware," in Workshop on CyberSecurity Experimentation and Test, 2011.

[34] J. Bluis and D. G. Shin, "Nodal distance algorithm: calculating a phylogenetic tree comparison metric," in Third IEEE Symposium on Bioinformatics and Bioengineering, 2003. 
[35] "A Brief History of Malware Obfuscation: Part 1 of 2," 2010. https://blogs.cisco.com/security/a_brief_history_ of_malware_obfuscation_part_1_of_2.

[36] “PEiD," 2017. https://www.aldeid.com/wiki/PEiD.

[37] "RDG Packer Detector," 2017. http://www.rdgsoft.net/.

[38] S. K. Debray, K. P. Coogan, and G. M. Townsend, "On the Semantics of SelfUnpacking Malware Code,” tech. rep., University of Arizona, AR, USA, 2008.

[39] W. Guizani, J.-Y. Marion, and D. Reynaud-Plantey, "Server-Side Dynamic Code Analysis," in International Conference on Malicious and Unwanted Software, 2009.

[40] S. Debray and J. Patel, "Reverse Engineering Self-Modifying Code: Unpacker Extraction," in Working Conference on Reverse Engineering, 2010.

[41] F. Guo, P. Ferrie, and T.-C. Chiueh, "A Study of the Packer Problem and Its Solutions," in International Symposium on Recent Advances in Intrusion Detection, 2008.

[42] B. Cheng, J. Ming, J. Fu, G. Peng, T. Chen, X. Zhang, and J.-Y. Marion, “Towards Paving the Way for Large-Scale Windows Malware Analysis: Generic Binary Unpacking with Orders-of-Magnitude Performance Boost," in ACM Conference on Computer and Communications Security, 2018.

[43] B. Schwarz, S. Debray, and G. Andrews, "Disassembly of Executable Code Revisited," in Working Conference on Reverse Engineering, October 2002.

[44] C. Linn and S. Debray, "Obfuscation of Executable Code to Improve Resistance to Static Disassembly," in ACM Conference on Computer and Communications Security, 2003.

[45] C. Kruegel, W. Robertson, F. Valeur, and G. Vigna, "Static Disassembly of Obfuscated Binaries," in USENIX Security Symposium, August 2004.

[46] J. Kinder and H. Veith, "Jakstab: A Static Analysis Platform for Binaries," in Computer Aided Verification, July 2008.

[47] J. Kinder, F. Zuleger, and H. Veith, "An Abstract Interpretation-Based Framework for Control Flow Reconstruction from Binaries," in International Conference on Verification, Model Checking, and Abstract Interpretation, January 2009.

[48] R. Paleari, L. Martignoni, G. Fresi Roglia, and D. Bruschi, "N-Version Disassembly: Differential Testing of x86 Disassemblers," in International Symposium on Software Testing and Analysis, 2010. 
[49] M. Zhang and R. Sekar, "Control Flow Integrity for COTS Binaries," in USENIX Security Symposium, 2013.

[50] D. Andriesse, X. Chen, V. van der Veen, A. Slowinska, and H. Bos, "An InDepth Analysis of Disassembly on Full-Scale x86/x64 Binaries," in USENIX Security Symposium, 2016.

[51] R. Wartell, Y. Zhou, K. W. Hamlen, M. Kantarcioglu, and B. Thuraisingham, "Differentiating Code from Data in x86 Binaries," in Joint European Conference on Machine Learning and Knowledge Discovery in Databases, 2011.

[52] T. Bao, J. Burket, M. Woo, R. Turner, and D. Brumley, "BYTEWEIGHT: Learning to Recognize Functions in Binary Code," in USENIX Security Symposium, 2014.

[53] E. C. R. Shin, D. Song, and R. Moazzezi, "Recognizing Functions in Binaries with Neural Networks," in USENIX Security Symposium, 2015.

[54] J. Caballero, N. M. Johnson, S. McCamant, and D. Song, "Binary Code Extraction and Interface Identification for Security Applications," in Network and Distributed System Security Symposium, 2010.

[55] D. Andriesse, A. Slowinska, and H. Bos, "Compiler-Agnostic Function Detection in Binaries," in European Symposium on Security \& Privacy, 2017.

[56] D. Gao, M. K. Reiter, and D. Song, BinHunt: Automatically Finding Semantic Differences in Binary Programs. Springer Berlin Heidelberg, 2008.

[57] M. Bourquin, A. King, and E. Robbins, "BinSlayer: Accurate Comparison of Binary Executables," in Program Protection and Reverse Engineering Workshop, ACM, 2013.

[58] "BinDiff," 2018. https://www.zynamics.com/bindiff.html.

[59] "Diaphora," 2018. http://diaphora.re/.

[60] B. H. Ng and A. Prakash, "Expose: Discovering potential binary code re-use," in Annual Computer Software and Applications Conference, IEEE, 2013.

[61] L. Luo, J. Ming, D. Wu, P. Liu, and S. Zhu, "Semantics-based Obfuscationresilient Binary Code Similarity Comparison with Applications to Software Plagiarism Detection," in International Symposium on Foundations of Software Engineering, ACM, 2014.

[62] Y. David and E. Yahav, "Tracelet-based Code Search in Executables," in Conference on Programming Language Design and Implementation, ACM, 2014. 
[63] J. Pewny, F. Schuster, L. Bernhard, T. Holz, and C. Rossow, "Leveraging Semantic Signatures for Bug Search in Binary Programs," in Annual Computer Security Applications Conference, ACM, 2014.

[64] J. Pewny, B. Garmany, R. Gawlik, C. Rossow, and T. Holz, "Cross-architecture bug search in binary executables," in IEEE Symposium on Security and Privacy, IEEE Computer Society, 2015.

[65] S. Eschweiler, K. Yakdan, and E. Gerhards-Padilla, "discovRE: Efficient CrossArchitecture Identification of Bugs in Binary Code," in Network and Distributed System Security Symposium, 2016.

[66] Y. David, N. Partush, and E. Yahav, "Statistical Similarity of Binaries," in Conference on Programming Language Design and Implementation, ACM, 2016.

[67] Q. Feng, R. Zhou, C. Xu, Y. Cheng, B. Testa, and H. Yin, "Scalable Graphbased Bug Search for Firmware Images," in ACM Conference on Computer and Communications Security, ACM, 2016.

[68] M. Chandramohan, Y. Xue, Z. Xu, Y. Liu, C. Y. Cho, and H. B. K. Tan, "BinGo: Cross-architecture cross-OS Binary Search," in International Symposium on Foundations of Software Engineering, ACM, 2016.

[69] H. Huang, A. M. Youssef, and M. Debbabi, "BinSequence: Fast, Accurate and Scalable Binary Code Reuse Detection," in Asia Conference on Computer and Communications Security, ACM, 2017.

[70] Q. Feng, M. Wang, M. Zhang, R. Zhou, A. Henderson, and H. Yin, "Extracting Conditional Formulas for Cross-Platform Bug Search," in Asia Conference on Computer and Communications Security, ACM, 2017.

[71] Y. David, N. Partush, and E. Yahav, "Similarity of Binaries Through Reoptimization," in Conference on Programming Language Design and Implementation, ACM, 2017.

[72] X. Xu, C. Liu, Q. Feng, H. Yin, L. Song, and D. Song, "Neural Network-based Graph Embedding for Cross-Platform Binary Code Similarity Detection," in ACM Conference on Computer and Communication Security, ACM, 2017.

[73] Y. David, N. Partush, and E. Yahav, "FirmUp: Precise Static Detection of Common Vulnerabilities in Firmware," in Conference on Architectural Support for Programming Languages and Operating Systems, ACM, 2018.

[74] P. Shirani, L. Collard, B. L. Agba, B. Lebel, M. Debbabi, L. Wang, and A. Hanna, "BINARM: Scalable and Efficient Detection of Vulnerabilities in Firmware Images of Intelligent Electronic Devices," in Conference on Detection of Intrusions and Malware, and Vulnerability Assessment, 2018. 
[75] B. Liu, W. Huo, C. Zhang, W. Li, F. Li, A. Piao, and W. Zou, “ $\alpha$ Diff: Crossversion Binary Code Similarity Detection with DNN," in Conference on Automated Software Engineering, ACM, 2018.

[76] X. Hu, T.-c. Chiueh, and K. G. Shin, "Large-scale Malware Indexing Using Function-call Graphs," in ACM Conference on Computer and Communications Security, ACM, 2009.

[77] J. Jang, D. Brumley, and S. Venkataraman, "BitShred: Feature Hashing Malware for Scalable Triage and Semantic Analysis," in ACM Conference on Computer and Communications Security, ACM, 2011.

[78] X. Hu, K. G. Shin, S. Bhatkar, and K. Griffin, "MutantX-S: Scalable Malware Clustering Based on Static Features," in USENIX Annual Technical Conference, 2013.

[79] T. Kim, Y. R. Lee, B. Kang, and E. G. Im, "Binary executable file similarity calculation using function matching," The Journal of Supercomputing, Dec 2016.

[80] C. Kruegel, E. Kirda, D. Mutz, W. Robertson, and G. Vigna, "Polymorphic Worm Detection Using Structural Information of Executables," in Symopsium on Recent Advance in Intrusion Detection, Springer Berlin Heidelberg, 2005.

[81] D. Bruschi, L. Martignoni, and M. Monga, "Detecting Self-mutating Malware Using Control-flow Graph Matching," in Detection of Intrusions and Malware and Vulnerability Assessment, Springer-Verlag, 2006.

[82] S. Cesare, Y. Xiang, and W. Zhou, "Control Flow-Based Malware Variant Detection," IEEE Transactions on Dependable and Secure Computing, vol. 11, no. 4, pp. 307-317, 2014.

[83] J. Ming, D. Xu, and D. Wu, "Memoized semantics-based binary diffing with application to malware lineage inference," in IFIP International Information Security Conference, Springer, 2015.

[84] Z. Wang, K. Pierce, and S. McFarling, "BMAT - A Binary Matching Tool," in In Second ACM Workshop on Feedback-Directed and Dynamic Optimization, 1999.

[85] T. Dullien and R. Rolles, "Graph-based Comparison of Executable Objects," in Symposium Sur La Securite Des Technologies De L'Information Et Des Communications, 2005.

[86] Y. Hu, Y. Zhang, J. Li, and D. Gu, "Cross-architecture binary semantics understanding via similar code comparison," in Conference on Software Analysis, Evolution, and Reengineering, IEEE, 2016. 
[87] Z. Xu, B. Chen, M. Chandramohan, Y. Liu, and F. Song, "SPAIN: Security Patch Analysis for Binaries Towards Understanding the Pain and Pills," in International Conference on Software Engineering, IEEE Press, 2017.

[88] U. Kargén and N. Shahmehri, "Towards Robust Instruction-level Trace Alignment of Binary Code," in International Conference on Automated Software Engineering, IEEE Press, 2017.

[89] S. seok Choi and S. hyuk Cha, "A survey of Binary similarity and distance measures," Journal of Systemics, Cybernetics and Informatics, pp. 43-48, 2010.

[90] S.-H. Cha, "Comprehensive Survey on Distance/Similarity Measures between Probability Density Functions," vol. 1, 012007.

[91] C. Collberg, S. Martin, J. Myers, and J. Nagra, "Distributed Application Tamper Detection via Continuous Software Updates," in 28th Annual Computer Security Applications Conference, 2012.

[92] A. Lakhotia, M. D. Preda, and R. Giacobazzi, "Fast Location of Similar Code Fragments Using Semantic 'Juice'," in Program Protection and Reverse Engineering Workshop, ACM, 2013.

[93] M. Bailey, J. Oberheide, J. Andersen, Z. M. Mao, F. Jahanian, and J. Nazario, "Automated Classification and Analysis of Internet Malware," in International Symposium on Recent Advances in Intrusion Detection, 2007.

[94] B. S. Baker, U. Manber, and R. Muth, "Compressing Differences of Executable Code," in ACMSIGPLAN Workshop on Compiler Support for System Software, 1999.

[95] A. Sæbjørnsen, J. Willcock, T. Panas, D. Quinlan, and Z. Su, "Detecting Code Clones in Binary Executables," in Symposium on Software Testing and Analysis, ACM, 2009.

[96] I. Santos, F. Brezo, J. Nieves, Y. K. Penya, B. Sanz, C. Laorden, and P. G. Bringas, "Idea: Opcode-Sequence-Based Malware Detection," in Symposium on Engineering Secure Software and Systems, Springer Berlin Heidelberg, 2010.

[97] B. Kang, T. Kim, H. Kwon, Y. Choi, and E. G. Im, "Malware Classification Method via Binary Content Comparison," in Research in Applied Computation Symposium, ACM, 2012.

[98] J. Ming, M. Pan, and D. Gao, "iBinHunt: Binary Hunting with Inter-procedural Control Flow," in International Conference on Information Security and Cryptology, Springer-Verlag, 2013. 
[99] W. Jin, S. Chaki, C. Cohen, A. Gurfinkel, J. Havrilla, C. Hines, and P. Narasimhan, "Binary function clustering using semantic hashes," in International Conference on Machine Learning and Applications, IEEE, 2012.

[100] W. M. Khoo, A. Mycroft, and R. Anderson, "Rendezvous: a Search Engine for Binary Code," in Working Conference on Mining Software Repositories, 2013.

[101] Y. R. Lee, B. Kang, and E. G. Im, "Function Matching-based Binary-level Software Similarity Calculation," in Research in Adaptive and Convergent Systems, ACM, 2013.

[102] M. R. Farhadi, B. C. M. Fung, P. Charland, and M. Debbabi, "BinClone: Detecting Code Clones in Malware," in International Conference on Software Security and Reliability, IEEE Computer Society, 2014.

[103] B. Ruttenberg, C. Miles, L. Kellogg, V. Notani, M. Howard, C. LeDoux, A. Lakhotia, and A. Pfeffer, "Identifying Shared Software Components to Support Malware Forensics," in Detection of Intrusions and Malware, and Vulnerability Assessment, Springer International Publishing, 2014.

[104] M. Egele, M. Woo, P. Chapman, and D. Brumley, "Blanket Execution: Dynamic Similarity Testing for Program Binaries and Components," in 23rd USENIX Security Symposium, 2014.

[105] L. Luo, J. Ming, D. Wu, P. Liu, and S. Zhu, "Semantics-based obfuscationresilient binary code similarity comparison with applications to software and algorithm plagiarism detection," IEEE Transactions on Software Engineering, no. 12, pp. 1157-1177, 2017.

[106] S. Alrabaee, P. Shirani, L. Wang, and M. Debbabi, "Sigma: A semantic integrated graph matching approach for identifying reused functions in binary code," 2015.

[107] J. Qiu, X. Su, and P. Ma, "Library functions identification in binary code by using graph isomorphism testings," in Conference on Software Analysis, Evolution and Reengineering, 2015.

[108] Y. Xiao, S. Cao, Z. Cao, F. Wang, F. Lin, J. Wu, and H. Bi, "Matching Similar Functions in Different Versions of a Malware," in Trustcom/BigDataSE/ISPA, 2016 IEEE, IEEE, 2016.

[109] N. Lageman, E. D. Kilmer, R. J. Walls, and P. D. McDaniel, "BinDNN: Resilient Function Matching Using Deep Learning," in International Conference on Security and Privacy in Communication Systems, Springer, 2016. 
[110] S. H. Ding, B. C. Fung, and P. Charland, "Kam1N0: MapReduce-based Assembly Clone Search for Reverse Engineering," in International Conference on Knowledge Discovery and Data Mining, ACM, 2016.

[111] Y. Hu, Y. Zhang, J. Li, and D. Gu, "Binary Code Clone Detection Across Architectures and Compiling Configurations," in International Conference on Program Comprehension, IEEE Press, 2017.

[112] L. Nouh, A. Rahimian, D. Mouheb, M. Debbabi, and A. Hanna, BinSign: Fingerprinting Binary Functions to Support Automated Analysis of Code Executables. Springer International Publishing, 2017.

[113] P. Shirani, L. Wang, and M. Debbabi, "BinShape: Scalable and Robust Binary Library Function Identification Using Function Shape," in Detection of Intrusions and Malware, and Vulnerability Assessment, Springer International Publishing, 2017.

[114] J. Ming, D. Xu, Y. Jiang, and D. Wu, "BinSim: Trace-based Semantic Binary Diffing via System Call Sliced Segment Equivalence Checking," in 26th USENIX Security Symposium, 2017.

[115] S. Alrabaee, P. Shirani, L. Wang, and M. Debbabi, "FOSSIL: A Resilient and Efficient System for Identifying FOSS Functions in Malware Binaries," IEEE Transactions on Privacy and Security, vol. 21, pp. 8:1-8:34, jan 2018.

[116] K. Redmond, L. Luo, and Q. Zeng, "A Cross-Architecture Instruction Embedding Model for Natural Language Processing-Inspired Binary Code Analysis," in Workshop on Binary Analysis Research, (San Diego, CA, USA), 2019.

[117] F. Zuo, X. Li, Z. Zhang, P. Young, L. Luo, and Q. Zeng, "Neural Machine Translation Inspired Binary Code Similarity Comparison beyond Function Pairs," in Network and Distributed System Security Symposium, 2019.

[118] S. H. H. Ding, B. C. M. Fung, and P. Charland, "Asm2vec: Boosting static representation robustness for binary clone search against code obfuscation and compiler optimization," in IEEE Symposium on Security and Privacy, (San Francisco, CA), IEEE Computer Society, 2019.

[119] T. Kamiya, S. Kusumoto, and K. Inoue, "CCFinder: a multilinguistic tokenbased code clone detection system for large scale source code," IEEE Transactions on Software Engineering, vol. 28, no. 7, pp. 654-670, 2002.

[120] H. Zhang and Z. Qian, "Precise and Accurate Patch Presence Test for Binaries," in 27th USENIX Security Symposium, (Baltimore, MD), 2018. 
[121] J. Crussell, C. Gibler, and H. Chen, "AnDarwin: Scalable Detection of Semantically Similar Android Applications," in European Symposium on Research in Computer Security, Springer Berlin Heidelberg, 2013.

[122] K. Chen, P. Liu, and Y. Zhang, "Achieving accuracy and scalability simultaneously in detecting application clones on android markets," in Proceedings of the 36th International Conference on Software Engineering, ACM, 2014.

[123] S. Forrest, S. A. Hofmeyr, A. Somayaji, and T. A. Longstaff, "A sense of self for unix processes," in IEEE Symposium on Security and Privacy, IEEE, 1996.

[124] E. Kirda, C. Kruegel, G. Banks, G. Vigna, and R. Kemmerer, "Behavior-based spyware detection.," in USENIX Security Symposium, 2006.

[125] M. Christodorescu, S. Jha, and C. Kruegel, "Mining Specifications of Malicious Behavior," in 6th Joint Meeting of the European Software Engineering Conference and the ACM SIGSOFT Symposium on The Foundations of Software Engineering, ACM, 2007.

[126] U. Bayer, P. M. Comparetti, C. Hlauschek, C. Kruegel, and E. Kirda, "Scalable, Behavior-Based Malware Clustering," in Network and Distributed System Security Symposium, 2009.

[127] G. Wicherski, "peHash: A Novel Approach to Fast Malware Clustering," in Conference on Large-scale Exploits and Emergent Threats: Botnets, Spyware, Worms, and More, USENIX Association, 2009.

[128] J. Kornblum, "Identifying Almost Identical Files Using Context Triggered Piecewise Hashing," Digital Investigation, vol. 3, pp. 91-97, Sept. 2006.

[129] J. Oliver, C. Cheng, and Y. Chen, "TLSH - A Locality Sensitive Hash," in Cybercrime and Trustworthy Computing Workshop, IEEE Computer Society, 2013.

[130] J. Newsome, B. Karp, and D. Song, "Polygraph: Automatically generating signatures for polymorphic worms," in IEEE Symposium on Security and Privacy, 2005.

[131] Z. Li, M. Sanghi, Y. Chen, M.-Y. Kao, and B. Chavez, "Hamsa: Fast signature generation for zero-day polymorphic worms with provable attack resilience," in IEEE Symposium on Security and Privacy, 2006.

[132] M. J. Rochkind, "The Source Code Control System," IEEE Transactions on Software Engineering, no. 4, pp. 364-370, 1975.

[133] W. F. Tichy, "RCS - A System for Version Control," Software: Practice and Experience, vol. 15, no. 7, pp. 637-654, 1985. 
[134] C. Reichenberger, "Delta Storage for Arbitrary Non-Text Files," in Proceedings of the 3rd ACM International Workshop on Software Configuration Management, 1991.

[135] "RTPatch," 1991. http://pocketsoft.com/rtpatch_binary_ diff.html.

[136] K. Coppieters, “A Cross-platform Binary Diff,” 1995.

[137] “Xdelta3," 1998. http://xdelta.org/.

[138] J. Jang, A. Agrawal, and D. Brumley, "ReDeBug: Finding Unpatched Code Clones in Entire OS Distributions," in IEEE Symposium on Security and Privacy, 2012.

[139] "DarunGrim," 2018. http://www.darungrim.org/.

[140] R. Perdisci, W. Lee, and N. Feamster, "Behavioral Clustering of HTTPBased Malware and Signature Generation Using Malicious Network Traces," in USENIX Symposium on Networked Systems Design and Implementation, 2010.

[141] M. Z. Rafique and J. Caballero, "FIRMA: Malware Clustering and Network Signature Generation with Mixed Network Behaviors," in Symposium on Research in Attacks, Intrusions and Defenses, (St. Lucia), October 2013.

[142] I. U. Haq, S. Chica, J. Caballero, and S. Jha, "Malware Lineage in the Wild," Elsevier Computer \& Security, vol. 78, pp. 347-363, 2018.

[143] Brumley, David and Poosankam, Pongsin and Song, Dawn and Zheng, Jiang, "Automatic patch-based exploit generation is possible: Techniques and implications," in IEEE Symposium on Security and Privacy, 2008.

[144] Z. Wang, K. Pierce, and S. McFarling, "BMAT - A Binary Matching Tool for Stale Profile Propagation," The Journal of Instruction-Level Parallelism, vol. 2, p. 2000, 2000.

[145] G. Myles and C. Collberg, "K-gram Based Software Birthmarks," in Symposium on Applied Computing, ACM, 2005.

[146] S. Choi, H. Park, H.-I. Lim, and T. Han, "A Static Birthmark of Binary Executables Based on API Call Structure," in Asian Computing Science Conference on Advances in Computer Science: Computer and Network Security, SpringerVerlag, 2007.

[147] L. Jiang and Z. Su, "Automatic Mining of Functionally Equivalent Code Fragments via Random Testing," in Symposium on Software Testing and Analysis, ACM, 2009. 
[148] V. Ganesh and D. L. Dill, "A Decision Procedure for Bit-vectors and Arrays," in Conference on Computer Aided Verification, Springer-Verlag, 2007.

[149] L. De Moura and N. Bjørner, "Z3: An Efficient SMT Solver," in Conference on Tools and Algorithms for the Construction and Analysis of Systems, SpringerVerlag, 2008.

[150] C. B. Zilles, "Master/slave Speculative Parallelization And Approximate Code," tech. rep., Univerisity of Wisconsin, USA, 2002.

[151] "VirusTotal," 2017. http: / / www.virustotal.com/.

[152] V. Roussev, "An Evaluation of Forensic Similarity Hashes," Digit. Investig., vol. 8, pp. S34-S41, Aug. 2011.

[153] F. Breitinger and H. Baier, "Similarity preserving hashing: Eligible properties and a new algorithm mrsh-v2," in Conference on Digital Forensics and Cyber Crime, Springer, 2012.

[154] F. Pagani, M. Dell'Amico, and D. Balzarotti, "Beyond Precision and Recall: Understanding Uses (and Misuses) of Similarity Hashes in Binary Analysis," in Conference on Data and Application Security and Privacy, ACM, March 2018.

[155] C. Linn and S. Debray, "Obfuscation of Executable Code to Improve Resistance to Static Disassembly," in ACM Conference on Computer and Communications Security, ACM, 2003.

[156] "ReadyNAS2014 Firmware Image v6.1.6," 2019. http://www . downloads.netgear.com/files/GDC/READYNAS-100/ ReadyNASOS-6.1.6-arm.zip.

[157] "DD-WRT2013 Firmware Image r21676," 2019. ftp://ftp.dd-wrt. com/betas/2013/05-27-2013-r21676/senao-eoc5610/ linux.bin.

[158] "Gartner says 8.4 billion connected things will be in use in 2017, up 31 percent from 2016VirusTotal." https: / /www.gartner.com/newsroom/ id/3598917.

[159] “Themida," 2018. https://www. oreans.com/themida.php/.

[160] "VMProtect," 2018. http: / / vmpsoft. com/products/vmprotect/.

[161] U. Bayer, P. M. Comparetti, C. Hlauschek, C. Kruegel, and E. Kirda, "Scalable, Behavior-Based Malware Clustering," in Network and Distributed System Security, 2009. 
[162] A. Dinaburg, P. Royal, M. Sharif, and W. Lee, "Ether: Malware Analysis via Hardware Virtualization Extensions," in ACM conference on Computer and Communications Security, 2008.

[163] “IDA," 2018. https://www.hex-rays.com/products/ida/.

[164] L. Luo, J. Ming, D. Wu, P. Liu, and S. Zhu, "Semantics-Based ObfuscationResilient Binary Code Similarity Comparison with Applications to Software Plagiarism Detection," in ACM SIGSOFT International Symposium on Foundations of Software Engineering, 2014.

[165] M. Sebastián, R. Rivera, P. Kotzias, and J. Caballero, "AVClass: A Tool for Massive Malware Labeling," in International Symposium on Research in Attacks, Intrusions and Defenses, September 2016.

[166] A. Mohaisen and O. Alrawi, "AV-Meter: An Evaluation of Antivirus Scans and Labels," in Detection of Intrusions and Malware, and Vulnerability Assessment, 2014.

[167] "PANDA: Platform for Architecture-Neutral Dynamic Analysis," 2017. https://github.com/moyix/panda.

[168] "CLOC," 2017. http: / / cloc.sourceforge.net/.

[169] C.-K. Luk, R. Cohn, R. Muth, H. Patil, A. Klauser, G. Lowney, S. Wallace, V. J. Reddi, and K. Hazelwood, "Pin: Building customized program analysis tools with dynamic instrumentation," in Conference on Programming Language Design and Implementation, ACM, 2005.

[170] M. Vrable, J. Ma, J. Chen, D. Moore, E. Vandekieft, A. C. Snoeren, G. M. Voelker, and S. Savage, "Scalability, Fidelity, and Containment in the Potemkin Virtual Honeyfarm," ACM SIGOPS Operating Systems Review, vol. 39, no. 5, pp. 148-162, 2005.

[171] C. Kreibich, N. Weaver, C. Kanich, W. Cui, and V. Paxson, "GQ: Practical containment for measuring modern malware systems," in ACM Internet Measurement Conference, 2011.

[172] M. M. Lehman and J. F. Ramil, "Rules and Tools for Software Evolution Planning and Management," Annals of software engineering, vol. 11, no. 1, pp. 1544, 2001.

[173] "SPEC CPU 2006 benchmark," 2017. https://www.spec.org/ cpu2006/.

[174] G. Lomow, J. Cleary, B. Unger, and D. West, "A Performance Study Of Time Warp," in SCS Multiconference on Distributed Simulation, 1988. 
[175] "PuTTY," 2017. ftp://ftp.chiark.greenend.org.uk/users/ sgtatham/.

[176] "FileZilla," 2017. https://sourceforge.net/projects/ filezilla/.

[177] "WinSCP," 2017. https://winscp.net/.

[178] “NotePad++," 2017. https://notepad-plus-plus.org/.

[179] "Process Hacker," 2017. http://processhacker.sourceforge. net $/$.

[180] "PANDA Malware Recordings," 2017. http://panda.gtisc.gatech. edu/malrec/.

[181] “AVClass," 2016. https://github.com/malicialab/avclass.

[182] “Angr: The Binary Analysis Framework," 2016. http://angr.io/.

[183] D. Brumley, I. Jager, T. Avgerinos, and E. J. Schwartz, "BAP: A binary analysis platform," in Conference on Computer Aided Verification, 2011.

[184] "BeaEngine," 2018. http://www.beaengine.org/.

[185] "Bitblaze: Binary Analysis For Computer Security," 2008. http:// bitblaze.cs.berkeley.edu/.

[186] "Boomerang decompiler," 2004. http://boomerang.sourceforge. net/.

[187] “Automated Malware Analysis," 2012. https: / / cuckoosandbox.org/.

[188] "Dyninst: Putting the Performance in High Performance Computing," 2009. http://www.dyninst.org/.

[189] “The LLVM Compiler Infrastructure,” 2004. http: / / l l vm.org/.

[190] "IDA Processor Support," 2019. http: / / www. fysnet. net/newbasic . htm.

[191] "McSema," 2012. https://github.com/trailofbits/mcsema/.

[192] "Pin - A Dynamic Binary Instrumentation Tool," 2005. https://software.intel.com/en-us/articles/ pin-a-dynamic-binary-instrumentation-tool.

[193] “QEMU: an open source processor emulator," 2006. http://www . qemu . org/. 
[194] "ROSE compiler infrastructure," 2000. http://www.rosecompiler. org/.

[195] "Valgrind," 2007. http://valgrind.org/.

[196] A. Srivastava, A. Edwards, and H. Vo, "Vulcan: Binary Transformation In A Distributed Environment," tech. rep., 2001. 